\title{
EFFECTS OF ATOMIC OXYGEN ON THE VACUUM-INDUCED MASS LOSS PROPERTIES OF A VARIETY OF SPACECRAFT MATERIALS
}

\author{
A Thesis \\ presented to \\ the Faculty of California Polytechnic State University, \\ San Luis Obispo
}

\author{
In Partial Fulfillment \\ of the Requirements for the Degree \\ Master of Science in Aerospace Engineering
}

by

Tyler Harty

December 2017 
(C) 2017

Tyler Harty

ALL RIGHTS RESERVED 


\section{COMMITTEE MEMBERSHIP}

TITLE:

AUTHOR:

DATE SUBMITTED: December 2017

COMMITTEE CHAIR: Kira Abercromby, Ph.D.

Associate Professor of Aerospace Engineering

COMMITTEE MEMBER: Timothy O'Donnell.

Manager at NASA JPL

COMmitTeE MEMBER: Amelia Greig, Ph.D.

Assistant Professor of Aerospace Engineering

COMMITTEE MEMBER: Jordi Puig Suari Ph.D.

Professor of Aerospace Engineering 


\begin{abstract}
Effects of Atomic Oxygen on the Vacuum-Induced Mass Loss Properties of a Variety of Spacecraft Materials

Tyler Harty
\end{abstract}

The space environment influences spacecraft design and material selection in many ways. Two aspects of the space environment that were of interest for this research are the vacuum and atomic oxygen (AO) environments. This project used the outgassing testing chamber and the AO chamber in the Cal Poly Space Environments lab to test multiple common spacecraft materials and determine whether $\mathrm{AO}$ affects the outgassing properties of those materials. This research has relevant applications in the design and material selection for spacecraft in low-Earth orbits. AO and outgassing are both known to be individual contributors of spacecraft material mass loss and degradation, but laboratory tests on the synergy are rare. ASTM E595 standardized test procedures were used to determine the Total Mass Loss (TML) and Collected Volatile Condensable Materials (CVCM) values for each material, at which point the test group of materials were subjected to AO exposure according to ASTM E2089 while the control group remained under similar vacuum and temperature conditions. Finally, all of the materials were subjected to a second ASTM E595 test. The results show a statistically significant effect of AO on some materials' outgassing properties. In particular, three of the four silicone materials tested showed a lower TML for the AO exposed group compared to the control group which can be explained by the glassification of silicone due to AO exposure. This explanation was confirmed by Fourier Transform Infrared (FTIR) spectroscopy. More testing is recommended to confirm the trends found during the testing and to re-test the materials whose wide variation in outgassing values prevented conclusions from being drawn. 


\section{ACKNOWLEDGMENTS}

Thanks to Dr. Amelia Greig for being a part of my committee and for being very helpful in troubleshooting issues in the lab when Dr. A was gone.

Thanks to Dr. Jordi Puig-Suari for being a part of my committee.

Thanks to Tim O'Donnell of JPL for being a part of my committee and much more. Tim facilitated the start of a student project with JPL that allowed me to get

funding for materials, parts and equipment for this research. Tim also organized a trip down to JPL that allowed me to present some of my research, get feedback for future work, and take some tours of the facilities at JPL. Tim was also a huge help in getting this research off the ground by getting suitable materials for testing.

Thanks to Nataly Chen of JPL who was kind enough to give me a tour of the outgassing lab that she runs at JPL. She also sent over data from her tests and provided many recommendations during the refurbishment of ELI.

Thanks to Cody Thompson, the lab tech, for being able and willing to drop whatever he was working on when an urgent problem arose in the lab. Cody was instrumental to the timeliness of getting data and finishing this research as he assisted with many small projects and troubleshot many issues beyond my abilities, especially electrical issues.

Thanks to Dan Goebel of JPL who was available to pass on some of his vast knowledge about vacuum systems, and who remotely assisted with the troubleshooting of the cryo pump when it broke, as well as provided recommendations for how to improve the ELI chamber.

Thanks to the Biomedical Engineering department and David Laiho, for graciously allowing me to borrow the helium leak detector for months on end while I troubleshot the leaks on ELI. 
Thanks to Dr. Trevor Harding for being available on short notice and very helpful in explaining how $\mathrm{AO}$ interacts with silicone on a molecular level, and for taking the time to show me how to use and analyze the results from the FTIR Spectrometer.

Thanks to Daniel Johnson, for being along for the grad school ride and for being a friend I could confer with whenever issues arose during the lab work and testing.

The biggest thanks to Dr. Kira Abercromby, for being a fantastically constructive and helpful mentor, friend, and thesis advisor. Dr. A was the inspiration behind this project and she was a constant source of motivation, knowledge, and sarcasm for which I am very grateful. 
$\begin{array}{lll}\text { Page } & \\ & & \end{array}$

LIST OF TABLES .......................... . . ix

LIST OF FIGURES . . . . . . . . . . . . . . . . . . . CHAPTER

1 Introduction . . . . . . . . . . . . . . . . . 1

1.1 Space Environments Background . . . . . . . . . . . . . . 1

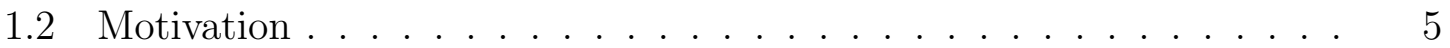

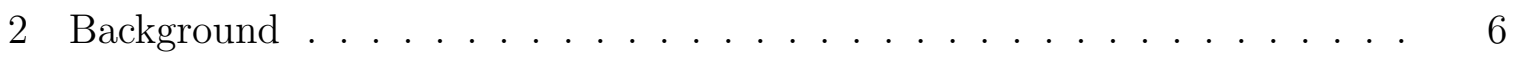

2.1 Atomic Oxygen . . . . . . . . . . . . . . . . 6

2.2 Atomic Oxygen with Charged Particles and UV . . . . . . . . . . . 13

2.3 Outgassing . . . . . . . . . . . . . . . . . 15

2.4 Outgassing with Atomic Oxygen and UV . . . . . . . . . . . . 20

3 Experimental Apparatuses . . . . . . . . . . . . . . . . 23

3.1 Apparatus $(\mathrm{ELI}) \ldots \ldots \ldots . \ldots \ldots$

3.2 Refurbishment (ELI) . . . . . . . . . . . . . . . 28

3.3 Apparatus $(\mathrm{MAX}) \ldots \ldots \ldots$. . . . . . . . . . . . . . 31

3.4 Microbalance Scale . . . . . . . . . . . . . . . . . . 36

3.5 Fourier Transform Infrared Spectrometer . . . . . . . . . . . . . . 39

4 Experimental Testing . . . . . . . . . . . . . . . . . 40

4.1 Outgassing Testing . . . . . . . . . . . . . . . 40

4.2 Atomic Oxygen Testing . . . . . . . . . . . . . . . . . . . 40

4.3 Synergistic Testing . . . . . . . . . . . . . . . . . . 42

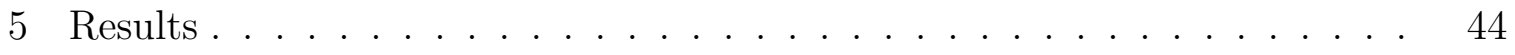

5.1 ELI Chamber Verification . . . . . . . . . . . . . . 44

5.2 MAX Chamber Verification . . . . . . . . . . . . . . . 48

5.3 Synergistic Testing . . . . . . . . . . . . . . . 51

5.4 FTIR Spectroscopy . . . . . . . . . . . . . . 58

6 Conclusion . . . . . . . . . . . . . . . . . . . 65

7 Future Work ........................... 69 
$7.1 \quad$ ELI Chamber $\ldots \ldots \ldots \ldots \ldots \ldots$

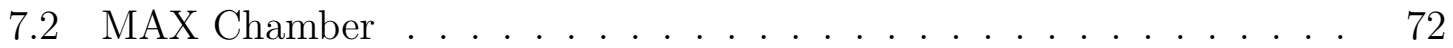

7.3 Microbalance Scale . . . . . . . . . . . . . . . . . . . . 74

7.4 Synergistic Testing . . . . . . . . . . . . . . . . 74

References . . . . . . . . . . . . . . . . . 76

APPENDICES

A Lessons Learned . . . . . . . . . . . . . . . . . . . . 81

B Additional Sample Pictures . . . . . . . . . . . . . . . . . . . . 89

C Procedures........................... 94

C.1 MAX Sample Preparation Procedure . . . . . . . . . . . . 94

C.2 MAX Pump Down Procedure . . . . . . . . . . . . . 95

C.3 MAX Operation Procedure . . . . . . . . . . . . . . . 96

C.4 MAX Shut-Down Procedure . . . . . . . . . . . . . . . . 97

C.5 ELI Sample Preparation . . . . . . . . . . . . . . . . . 98

C.6 ELI Pump Down Procedure with Cryo Pump Off . . . . . . . . . . 100

C.7 ELI Pump Down Procedure with Cryo Pump On . . . . . . . . . . . 101

C.8 ELI Operation Procedure . . . . . . . . . . . . . . . . . . . 102

C.9 ELI Shut Down Procedure . . . . . . . . . . . . . . . . . . 102

C.10 NASA JPL Modified ASTM E595 Procedure . . . . . . . . . . . . 103

C.11 Leak Detector Procedure . . . . . . . . . . . . . . . . . . . 107

C.12 Cryo Pump Compressor Procedures . . . . . . . . . . . . . . . . 109

C.13 Anti-Static Gun Procedure . . . . . . . . . . . . . . . . . . 111 


\section{LIST OF TABLES}

Table

Page

2.1 Reaction Efficiencies for Standard Spacecraft Materials [16] . . . . 11

2.2 Activation Energies and Time Dependencies for Three Mechanisms of Outgassing [37]. . . . . . . . . . . . . . . . . . . 16

5.1 Materials list with TML data. Asterisk denotes a similar material. . 45

5.2 Materials list with CVCM data. Asterisk denotes a similar material. 47

5.3 Independent Samples t-test Results for TML data . . . . . . . . . 57

5.4 Independent Samples t-test Results for CVCM data . . . . . . . . . 58 


\section{LIST OF FIGURES}

Figure

2.1 Atmospheric particle number density relative to altitude according to the NRLMSISE-00 atmosphere model. . . . . . . . . . . . . . . .

2.2 Monthly AO fluence for the RAM direction in a $400 \mathrm{~km}$ circular orbit with $0^{\circ}$ inclination from Jan 1st 1994 to Dec 31st 2016. . . . . . . .

2.3 Polar plot of relative atomic oxygen flux as a function of the angle between the ram direction and the normal of the arrival surface for a LEO spacecraft in a $400 \mathrm{~km}$ orbit at 28.5 inclination and $1000 \mathrm{~K}$

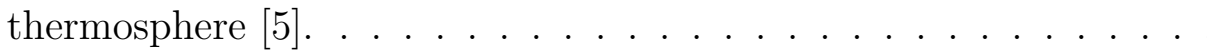

2.4 Scanning electron microscope photograph showing surface roughness (pits and cones) of Chlorotrifluoroethylene after AO exposure [14] .

2.5 Effect of AO on the outgassing of S0383-70 and ELA-SA-401 O-rings $[10]$

3.1 Outgassing testing chamber with water cooling unit and compressor. 24

3.2 Full Schematic of ELI chamber. . . . . . . . . . . . 26

3.3 ELI Chamber Test Stand Close-Up . . . . . . . . . . . . . . 27

3.4 Top, powered plate in MAX comprised of circular electrode and square dark space shield. [12] . . . . . . . . . . . . . . . 33

3.5 Full schematic of MAX chamber. . . . . . . . . . . . . . 34

4.1 Synergistic Test Procedure . . . . . . . . . . . . . . . 43

5.1 Sample Bar Temperature by Location . . . . . . . . . . . . . . . . . 44

5.2 Kapton tape, 2 mil Kapton, 3 mil Kapton (left to right) before and after AO exposure with a Kapton HN witness sample in the top center 49

5.3 Tflex silicone thermal gap filler (grey rectangles) and EN-11 polyurethane (orange cubes) before and after $\mathrm{AO}$ exposure with a Kapton $\mathrm{HN}$ witness sample in the top center. . . . . . . . . . . . . 50

5.4 EA 9360 Samples TML and CVCM Outgassing Results . . . . . . . 52

5.5 Silicone Materials TML and CVCM Outgassing Results for AO Exposed Samples in the Final Test Compared to Control Samples in the Final Test . . . . . . . . . . . . . . . . . . . . 
5.6 Retested Samples TML and CVCM Outgassing Results for All Samples in the First Initial Test Compared to All Samples in the Second Initial Test . . . . . . . . . . . . . . . . . .

5.7 Silicone Materials TML and CVCM Outgassing Results for all Samples in the Initial Test Compared to Control Samples in the Final

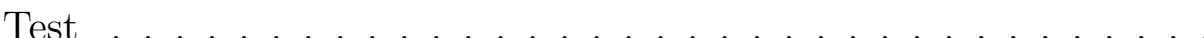

5.8 General Polydimethylsiloxane Silicone Transmittance Response to FTIR [28] . . . . . . . . . . . . . . . . . . . . . . . . . . . . . . . . . . . 59

5.9 RTV 566 Silicone Transmittance Response to FTIR . . . . . . . . 60

5.10 CV 2566 Silicone Transmittance Response to FTIR . . . . . . . . . 61

5.11 Zoom View of CV 2566 Silicone FTIR peak at a wavenumber of 1258

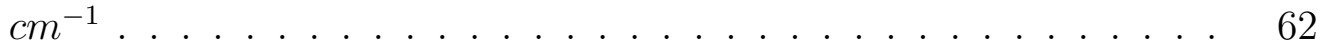

5.12 Zoom View of Adjusted CV 2566 Silicone FTIR peak at a wavenumber of $1258 \mathrm{~cm}^{-1} \ldots \ldots \ldots \ldots \ldots \ldots$

5.13 Zoom View of RTV 566 Silicone FTIR peak at a wavenumber of 1258 $\mathrm{cm}^{-1} \ldots \ldots \ldots \ldots \ldots \ldots \ldots$

5.14 Zoom View of Adjusted RTV 566 Silicone FTIR peak at a wavenumber of $1258 \mathrm{~cm}^{-1} \ldots \ldots \ldots \ldots \ldots \ldots$

5.15 Tflex Silicone Transmittance Response to FTIR . . . . . . . . . . . 64

A.1 NASA JPL outgassing chamber for ASTM E595 Testing. . . . . . 88

B.1 Graphite composite disks and 2 mil Kapton strips before and after AO exposure with a Kapton HN witness sample in the top center .

B.2 3 mil Kapton strips and EA 9360 Loctite adhesive (blue pieces) before and after $\mathrm{AO}$ exposure with a Kapton $\mathrm{HN}$ witness sample in the top center . . . . . . . . . . . . . . . . .

B.3 NuSil R-2141 two-part silicone adhesive (white samples on aluminum boats) and CV-2566 silicone rubber (red rectangles) before and after AO exposure with a Kapton HN witness sample in the top center .

B.4 EN-11 Polyurethane samples untested (yellow transparent large piece), outgassed (top right transparent orange cube), and outgassed \& AO exposed (top left translucent orange cube) . . . . . . . . . . . 91

B.5 Tflex samples AO exposed and outgassed (left), and outgassed (right) 92

B.6 RTV 566 silicone rubber samples untested (right), outgassed and AO exposed (center column), and outgassed (left column) . . . . . . 92

B.7 CV-2566 silicone rubber samples untested (right), outgassed and AO exposed (center column), and outgassed (left column) . . . . . . . . 
B.8 EA 9360 Loctite adhesive samples untested (left), outgassed and AO exposed (center column), and outgassed (right column) . . . . . .

B.9 NuSil R-2141 Silicone two-part adhesive samples original uncured material (left), outgassed and AO exposed (center column), and outgassed (right column) . . . . . . . . . . . . . . . . . 93

C.1 Spray Nozzle for use with Helium Leak Detector . . . . . . . . . . 108 


\section{Chapter 1}

\section{INTRODUCTION}

\subsection{Space Environments Background}

The space environment is defined as the ambient environment around a spacecraft or other object of interest, as well as the environment created by the spacecraft/object itself. There are many different aspects of the space environment, only some of which are of concern for a specific location in space. These aspects include the radiation, plasma, particulate, and neutral environments, each of which individually can result in interesting effects on spacecraft materials, units, and subsystems. These aspects of the environment are not independent, however, as they can combine to create synergistic effects that can be much more damaging than the individual effects alone.

The radiation environment refers to alpha, beta, gamma, and galactic cosmic ray (GCR) radiation that a spacecraft is exposed to from the Sun, the ambient universe, and from any on-board source like a radioisotope thermoelectric generator (RTG). The latter of the four external radiation sources does not originate from our Sun, and the GCR particles can be heavy ions from common elements like iron. These GCR particles can typically have energies that are orders of magnitude higher than even the highest energy gamma rays. GCR particles are generally observed at a much lower frequency than the other types of radiation, but their high energies can cause vastly more damage that they must still be accounted for. Alpha particles are essentially a helium nucleus, consisting of two protons and two neutrons. This means they are relatively heavy and slow moving particles compared to beta and gamma radiation. Beta particles are highly energized electrons that move at high speeds. Gamma radiation is a photon in the highest energy portion of the electromagnetic spectrum. 
Since the alpha, beta, and gamma radiation can vary significantly in energy, the solar radiation environment is generally categorized into three types: solar wind, trapped radiation, and solar particle events (SPE) [37]. Without a dense atmosphere of particles to protect them, spacecraft are exposed to much more radiation than anything on the surface of the Earth. This radiation damage can accumulate over time to destroy sensitive electronics and cause serious cell reproduction issues in humans. The radiation exposure that a spacecraft may see is not the same for every location in the solar system. The Van Allen Belts are a particularly notorious location for harsh radiation exposure due to Earth's magnetic field concentrating radiation particles there. The radiation environment also includes ultraviolet (UV) radiation that does not have enough energy to penetrate deep into the spacecraft, but does deteriorate the surface, which can cause discoloration and changes in absorptivity and reflectivity $[32]$.

The plasma environment refers to the most abundant state of matter in the universe including neutral and charged particles whose energies are lower than radiation particles. Space is not empty; in fact, it is full of protons, positively charged ions, electrons, and even neutral particles that together make up the plasma in space. These particles originate from our Sun and the universe flying around at relatively high speeds compared to spacecraft orbital speeds. The sun releases a lot of ions while electrons are flying in from all over the universe (at speeds much higher than the ions). These ions and electrons are constantly running into spacecraft where, depending on their energy, they either stick to the surface materials or they penetrate into the interior of the spacecraft, potentially depositing themselves onto sensitive electronics. These charges accumulate over time and spacecraft can build up a charge relative to the more or less neutral plasma if it is being hit with more ions than electrons, or vice versa. For example, in geostationary orbit it is common for spacecraft to accumulate a charge of a few kilovolts negative [32]. There is no actual ground 
for a spacecraft to bleed this charge off to so it must do a good job of distributing the charge evenly, otherwise certain components and materials can charge up to create huge potential differences that can result in arcing which will destroy or disrupt sensitive electronics [32]. Some of these issues can be prevented through shielding in the same way radiation is dealt with. For the particles that will undoubtedly make it through the shielding, other tactics must be used including designing robust grounding schemes and applying conductive coatings to dielectrics. In addition, one must ensure there are no units, components, or materials unintentionally floating relative to ground within the spacecraft.

The particulate environment refers to micro meteoroids and orbital debris (MMOD). In general, micro meteoroids are meteors with masses smaller than a gram [32]. These objects can have considerable velocities relative to spacecraft and can cause significant damage upon impact. Even an object on the microscopic scale could destroy or totally disable a spacecraft if it were, for example, to puncture a tank. Orbital debris refers to the man-made objects that are space junk or other small pieces of debris from former satellites or launch vehicles. Orbital debris is primarily concentrated near the Earth in low-Earth orbits (LEO) and while it does not have the same relative velocities that micro meteorites can have, it is far more abundant, especially in LEO. The U.S. Space Surveillance Network, a branch of the Department of Defense, tracks all objects larger than $10 \mathrm{~cm}$ in diameter in LEO and they track all objects larger than one $\mathrm{m}$ in GEO [27]. In addition, they forecast when two objects have a high probability of colliding in the near future and they alert satellite operators of the need to maneuver to avoid a collision. However, all objects smaller than those thresholds are not tracked and thus design considerations need to be made for particles of those sizes. Thankfully there are not many micro meteoroids larger than a fraction of a centimeter in diameter and those that are smaller can be stopped by shielding. For determining the required design thickness for the shielding, probability of no pene- 
tration (PNP) and probability of no critical failure (PNCF) are analyzed to show a high probability that the spacecraft will survive the particulate environment. Orbital debris poses a much larger problem in LEO than in GEO simply because objects in GEO are mostly all in the same orbit, separated by true anomaly so there is not a high chance of two spacecraft colliding with one another. In LEO on the other hand, spacecraft are in all kinds of orbits with significantly varying eccentricity, inclination, ascending node line, etc. Lower stage rocket bodies also present a problem and as a result, NASA is funding companies like Space Systems Loral (SSL) to look into the feasibility of re-purposing rocket bodies for human habitats [36].

The neutrals environment refers to particles, or the lack thereof, wherever a spacecraft is. In LEO there are still remains of the Earth's atmosphere as the density decreases exponentially with altitude. The most common particles at these altitudes are oxygen atoms referred to as Atomic Oxygen (AO) as UV from the Sun photochemically disassociates $\mathrm{O}_{2}$ into lone oxygen. AO molecules are very reactive with many types of materials, especially polymers. Beyond LEO there are not many particles because as Earth's atmosphere density decreases to insignificance. Here the neutrals environment is generally just the vacuum environment or any particles that the spacecraft itself is producing. The vacuum environment results in a interesting phenomenon known as outgassing, where air molecules trapped on the surface or inside a material will try to escape when the material is under vacuum. This can result in volatile compounds being released and contaminating other materials within their line of sight, posing a significant problem for precise sensors like optics lenses or mirrors. For this reason, standards like ASTM E595 and ASTM E1559 have been developed to qualify a material for space applications $[1,2]$. These standards define recommended acceptable thresholds for outgassing properties like total mass loss (TML) and collected volatile condensable materials (CVCM) that will be discussed later. Another aspect of the neutrals environment is drag. Even though there is a low 
density of particles at high altitudes, spacecraft travel at fast enough speeds that the particles they do run into impart a significant momentum exchange such that it will cause the spacecraft to slow and eventually de-orbit at lower LEO altitudes. During launch, pressure differentials are a concern as air within the spacecraft tries to escape to equalize with the rapidly decreasing external air density during the ascent of the launch vehicle. To handle this, designers must ensure proper venting holes and slits in thermal blankets, honeycomb paneling, and any other structure not designed to be a pressure vessel.

\subsection{Motivation}

Any individual aspect of the space environment can pose many significant design challenges for aerospace engineers, and some effects have been shown to react synergistically and produce more harm than the sum of the two effects individually. While synergistic effects between UV radiation and AO, as well as UV and outgassing have been studied, there is not much literature on any potential synergistic effects between AO and outgassing except for the work of Eli Gurnee [14]. However, his research had the flaws of a low precision scale and testing very few materials which resulted in the inability to make strong conclusions from the results. This research seeks to address those issues in order to better understand these aspects of the space environment. The materials tested included kapton coated films, silicones, adhesives, and a graphite composite. There was a wide range of known erosion rates due to AO among the materials. This research has potentially significant implications. If a layered material has the top layer exposed to and eroded from AO exposure and then outgasses significantly differently than it did before the AO exposure then that would mean actual designs using that material would be either over-designed or over-contaminating the neighboring surfaces. Both cases should be avoided, but the latter is much worse. 
Chapter 2

BACKGROUND

\subsection{Atomic Oxygen}

The composition of the atmosphere is not consistent with altitude. On the Earth's surface there is a majority of Nitrogen $\left(N_{2}\right)$ making up about $78 \%$ of the particles, followed by $21 \%$ Oxygen $\left(\mathrm{O}_{2}\right)$ and the remaining $1 \%$ made up of other elements. At altitudes where spacecraft orbit, the Nitrogen concentration begins to die off and $\mathrm{O}_{2}$ would be the main component, however, UV radiation from the Sun has a high enough energy to photochemically break the $5.12 \mathrm{eV}$ bond energy of $\mathrm{O}_{2}$. This radiation has wavelengths less than 243 nanometers and does not reach Earth's surface because it is absorbed by the atmosphere. It is only able to penetrate down to about $60 \mathrm{~km}$ in altitude [14]. The AO concentration increases with altitude from there as the UV radiation increases and the total density of all particles decreases which means the mean free path increases, thus making it less likely that an $\mathrm{AO}$ atom will collide with another AO atom to form $\mathrm{O}_{2}$ or $\mathrm{O}_{3}$ (Ozone). The AO concentration continues to increase until it becomes the predominant atmospheric particle at about $175 \mathrm{~km}$. This remains true until $600 \mathrm{~km}$ in altitude at which point Helium takes over. This is visualized in fig. 2.1.

The actual number densities in this figure vary significantly with Solar cycle which is a function of the year and day of year. It also fluctuates with the geomagnetic index, as higher Solar or geomagnetic activity causes the atmosphere to expand. The primary indicator of Solar activity is the f10.7 value which is the measured solar intensity for the wavelength of $10.7 \mathrm{~cm}$ at $1 \mathrm{AU}$ from the Sun. Figure 2.1 was created with an f10.7 of 90 and an $A_{p}$ of 15 on January 1 st at $0^{\circ}$ latitude and $0^{\circ}$ longitude. The solar 


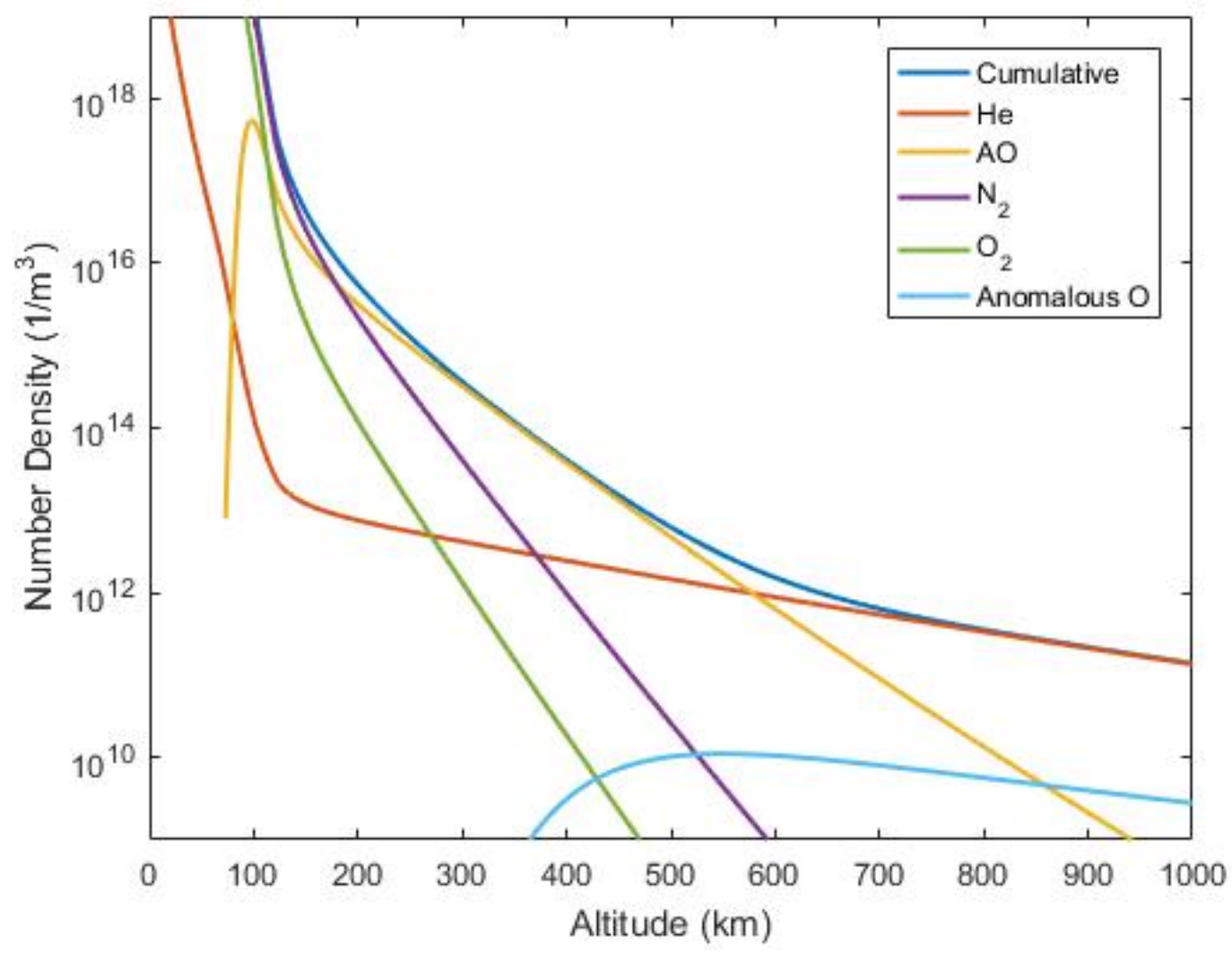

Figure 2.1: Atmospheric particle number density relative to altitude according to the NRLMSISE-00 atmosphere model.

activity tends to vary on a roughly 11 year cycle which can be seen in fig. 2.2 as it affects AO fluence. This plot was generated using the MSISE-00 atmospheric model with a $+2 \sigma$ estimate for the f10.7 solar flux, so the figure is not actual fluence data.

As mentioned earlier, this atomic oxygen erodes materials quickly for multiple reasons. The first reason is that the oxygen atom itself is highly reactive due to its six valence electrons; it can form strong bonds with carbon, oxygen, silicon, and other elements in order to fill its valence shell with eight electrons. It poses a special problem for spacecraft, however, due to the physics of an orbit near the Earth. A satellite in a $400 \mathrm{~km}$ circular orbit has a speed of $7.67 \mathrm{~km} / \mathrm{s}$ in order to maintain the perpetual free-fall that keeps it in orbit. This results in a high impact velocity of the oxygen atoms with the spacecraft ram direction surfaces. The AO is not stationary 


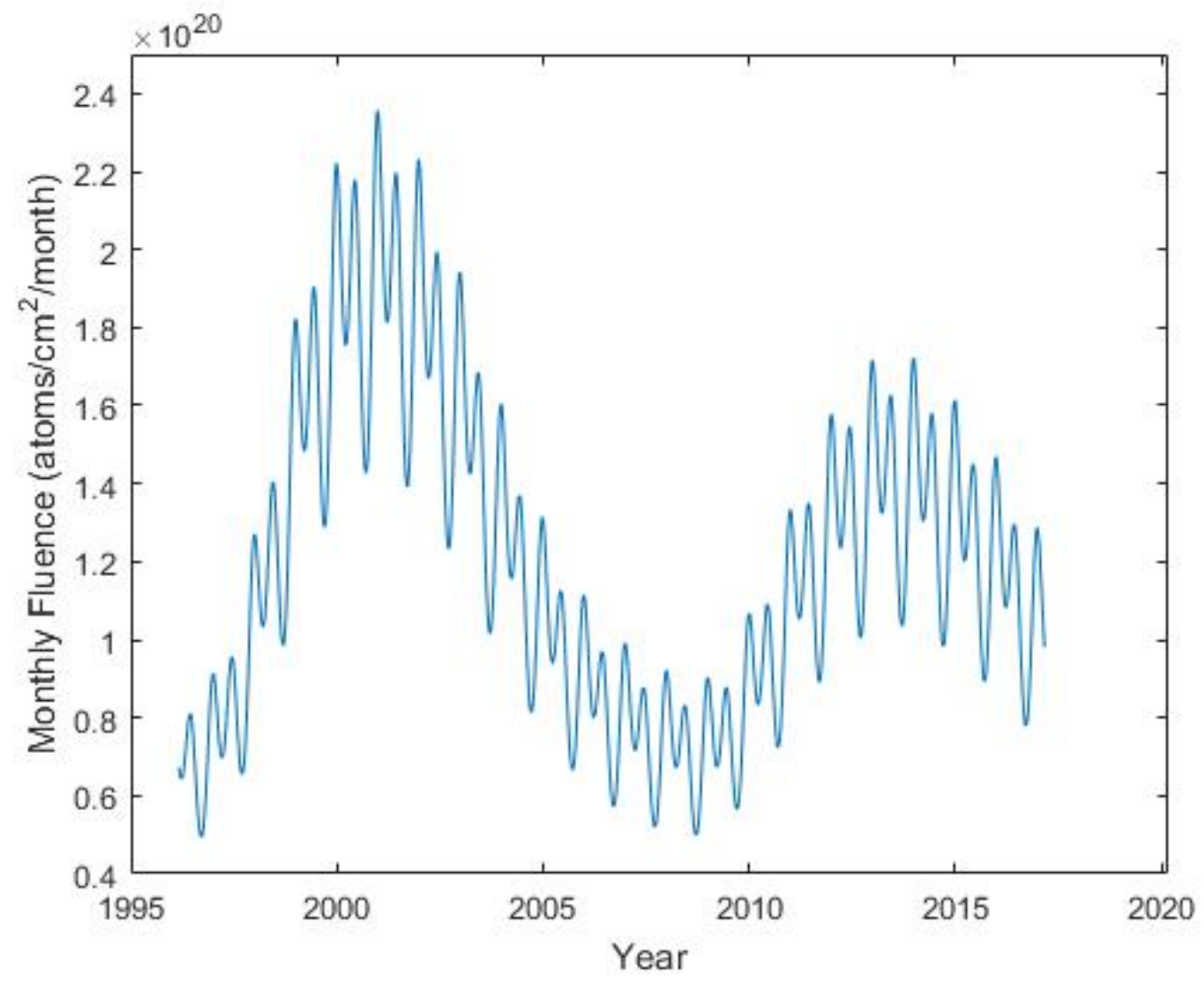

Figure 2.2: Monthly AO fluence for the RAM direction in a $400 \mathrm{~km}$ circular orbit with $0^{\circ}$ inclination from Jan 1st 1994 to Dec 31st 2016.

relative to the inertial frame; it has some velocity associated with the rotation of the Earth which can be as high as $0.5 \mathrm{~km} / \mathrm{s}$ at a latitude of $0^{\circ}$. As a result, there can be some variation in the impact energy of the $\mathrm{AO}$ particles depending on the orbit and the position of the satellite in that orbit. The AO itself also has some inherent thermal velocity that is a function of the temperature of the thermosphere. All of this amounts to an $\mathrm{AO}$ impact energy of $4.5 \pm 1 \mathrm{eV}$ on the ram direction surfaces of a spacecraft in a $400 \mathrm{~km}$ circular orbit [14]. However, ram surfaces are not the only ones affected, as the high variation in $\mathrm{AO}$ thermal energy and spacecraft velocity relative to the Earth's co-rotation cause other surfaces to be exposed. Figure 2.3 shows the relative flux of surfaces at all angles relative to ram. The figure shows that even the anti-ram face receives some flux, although it is orders of magnitude smaller. 


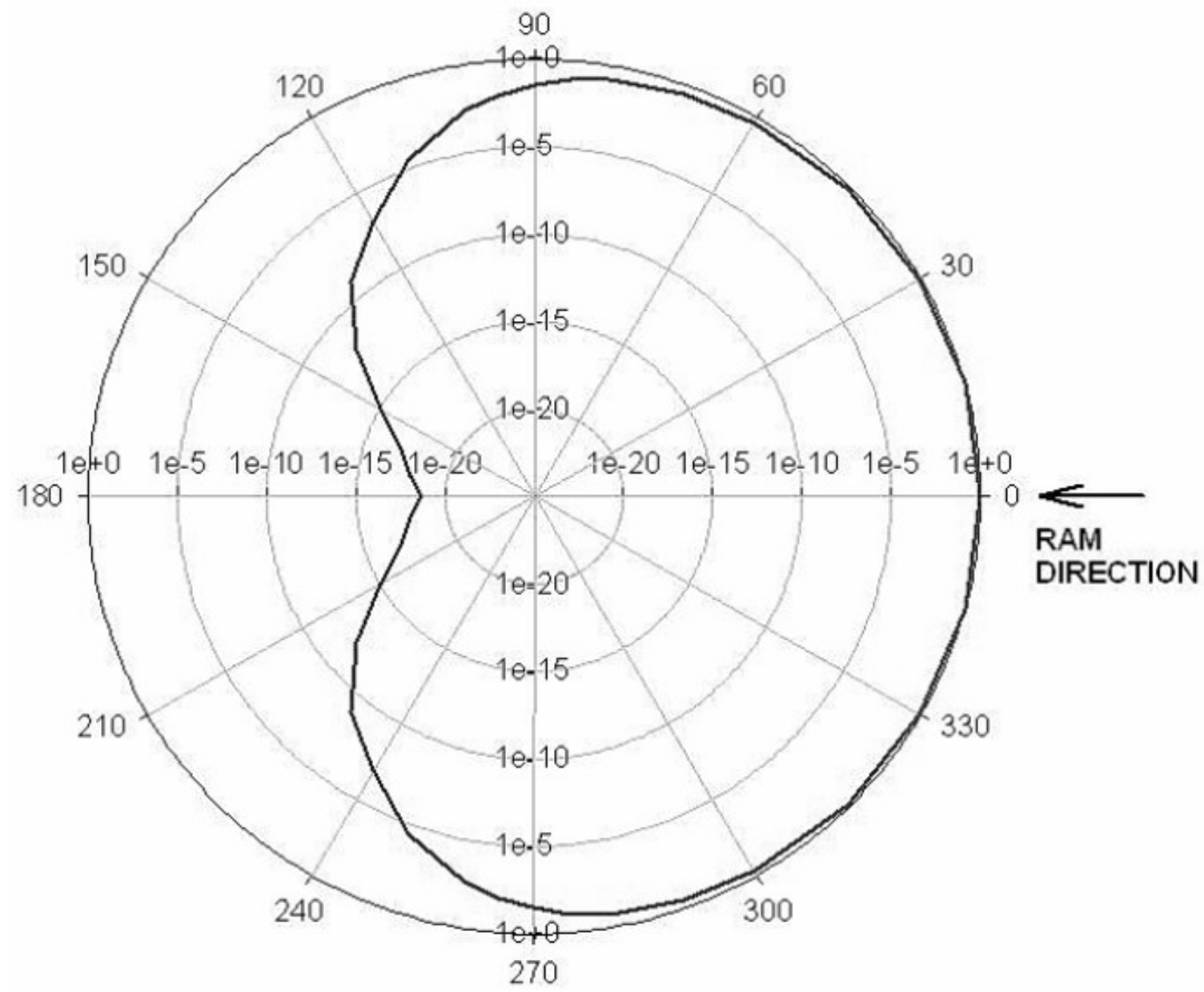

Figure 2.3: Polar plot of relative atomic oxygen flux as a function of the angle between the ram direction and the normal of the arrival surface for a LEO spacecraft in a $400 \mathrm{~km}$ orbit at 28.5 inclination and $1000 \mathrm{~K}$ thermosphere [5].

Material degradation due to AO has two main sources: erosion caused by the formation of oxides that do not adhere to the material's surface and erosion caused by the formation of volatile oxides [14]. An example of the former would be the reaction of $\mathrm{AO}$ and silver, as silver oxides do not adhere to the surface of the the bulk material. An example of a formation of a volatile oxide would be the reaction between $\mathrm{AO}$ and graphite to form volatile carbon-monoxide. When testing a material for its susceptibility to $\mathrm{AO}$, the important thing to quantify is the reaction efficiency which describes the volume of material removed from each $\mathrm{AO}$ atom that hits the 
surface. This constant can be used to predict a total thickness of the material that would be eroded when exposed to a given $\mathrm{AO}$ environment for a set amount of time. The reaction efficiency is usually expressed in the units $\mathrm{cm}^{3} /$ atom and is used in the equation

$$
\Delta m=\rho_{m} * R E * \phi * A * t
$$

where $\Delta m$ is mass loss in grams, $\mathrm{RE}$ is the reaction efficiency in $\mathrm{cm}^{3} /$ atom, $\rho_{m}$ is the density of the material in $\frac{g}{\mathrm{~cm}^{3}}, \phi$ is the flux of $\mathrm{AO}$ in $\frac{\text { atoms }}{\mathrm{cm}^{2} * s}, A$ is the material's exposed surface area in $\mathrm{cm}^{2}$, and $t$ is the total time the material is exposed to AO in $s$. Many on-orbit and ground tests have been conducted to determine the baseline reaction efficiency of many materials, however the actual reaction efficiency of a material on orbit can vary slightly from this estimate due to factors like temperature, AO flux, UV radiation, surface contamination, charged particle flux, and others [14].

$\mathrm{AO}$ is very reactive with many materials that are commonly chosen for the design of a spacecraft for various reasons. Some of these materials include most polymers like Kapton (used in multi-layer insulation (MLI) blanketing, tapes, and other applications where its thermal properties are desirable), carbons (used in graphite composite structures), silicones (used as bonding agents or as thermal barriers between units), and soft metals like silver (used as interconnect material between solar cells). A list of some materials and their reaction efficiencies are shown in table 2.1.

One issue that AO causes with polymers is a surface texturing that is due to pits and cones formed by high relative velocity between the spacecraft and the AO particles, typically on the ram-facing surface as shown in fig. 2.4. This change in surface texture tends to decrease specular transmittance, increase diffuse reflectance, as well as result in changes to thermal emittance and absorptivity of the material. This can be a significant issue as many polymers are chosed for their thermal properties 
Table 2.1: Reaction Efficiencies for Standard Spacecraft Materials [16]

\begin{tabular}{|l|l|l|l|}
\hline Material & $\begin{array}{l}\text { RE } \\
\left.* 10^{-30}\right)\end{array} \quad\left(m^{3} /\right.$ atom & Material & $\begin{array}{l}\text { RE } \\
\left.* 10^{-30}\right)\end{array}$ \\
\hline Kapton HN & 2.8 & Carbon & 1.2 \\
\hline Kapton H & 3.0 & Polystyrene & 1.2 \\
\hline Mylar & 3.4 & Polymide & 3.3 \\
\hline Platinum & 0 & Silver & 10.4 \\
\hline Polyethylene & 3.7 & Copper & 0.05 \\
\hline Teflon FEP and FE & $<0.1$ & Gold & 0 \\
\hline
\end{tabular}

for use in MLI blankets which are exterior to the spacecraft and exposed to AO. MLI also often has coated polymer materials which are susceptible to undercutting. Undercutting is where an exterior coating or surface of a material may be resistant to $\mathrm{AO}$, but due to the manufacturing or handling processes small defects in the coating expose the underlying material allowing AO to significantly erode it.

The main issue with carbon erosion due to AO has nothing to do with its thermal properties, but rather the structural degradation. Carbon based fibers are commonly used in composite structures to replace aluminum which is not affected by AO. Shin et al in [35] determined that $\mathrm{AO}$ is the most harmful aspect of the space environment for organic matrix composites. The AO exposure of the composite solar array structural panel tested showed dimensional changes, mass loss, and mechanical property degradation as well as a decrease in strength and stiffness of graphite based composites with an epoxy resin.

The effect of $\mathrm{AO}$ on silicones is an interesting one as it does not involve material removal. Silicones are generally repeating strings of alternating silicon and oxygen 


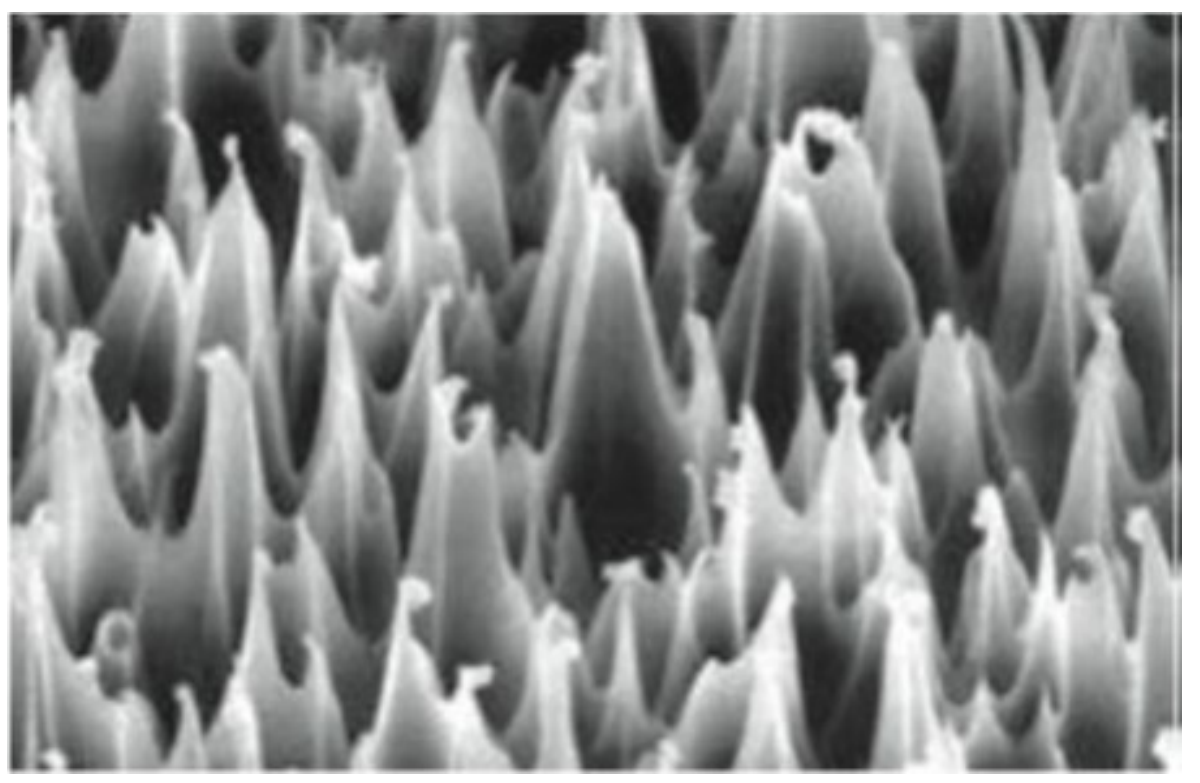

Figure 2.4: Scanning electron microscope photograph showing surface roughness (pits and cones) of Chlorotrifluoroethylene after AO exposure [14]

(known as siloxane) with other elements sometimes present as well. The AO has enough energy to break the bond of the silicon-oxygen and form silicon-dioxide which is silica (also known as glass). This layer of silica on top of the silicone has a number of different properties due to the transformation from rubber to glass, including changes in thermal properties, stiffness, and increased resistance to further AO exposure. Depending on the design application, this can be a benefit. Pre-treating some silicones with $\mathrm{AO}$ exposure can result in them resisting further $\mathrm{AO}$ degradation, so long as the surface silica does not crack and flake away from the underlying silicone. However, if the rubber silicone material is important to be maintained, such as in seals, AO exposure will not be beneficial, as it results in increased leak rates of silicone seals $[31]$.

Soft metals like silver and osmium are some of the only metals that are affected by AO exposure, however, the effects can be drastic as silver has a significantly higher reaction efficiency than copper as shown in table 2.1. Silver also erodes significantly 
differently than polymers. Instead of microscopic pits and cones, the AO creates a silver-oxide on the surface that continually flakes away and exposes more silver underneath. This flaking is referred to as spalling and it has been the cause of multiple solar array failures as the interconnectors between cells are made of silver $[12,11,37]$.

When it comes to mitigation of degradation due to AO, many materials can be coated in a thin film like indium tin oxide (ITO), $\mathrm{SiO}_{2}, \mathrm{Al}_{2} \mathrm{O}_{3}$, germanium, aluminum, gold, or some silicones as each of these either have or form a stable oxide that prevents further reactions with AO. As mentioned, the application process must be precise as small defects will result in undercutting. Generally, effective coatings are about 100 $\mathrm{nm}$ or less, as thicker coatings are prone to cracking. Some materials' surfaces can also be altered to be more AO resistant through the addition of metal atoms like silicon. A polymer that already is designed to have metal atoms within it is siloxane. The metal ions help form stable oxides in the presence of AO [14].

\subsection{Atomic Oxygen with Charged Particles and UV}

Few facilities have the capabilities to investigate how secondary effects can contribute to material degradation in ground based AO chambers. As a result, there have not been many experiments that have quantified the effects of flux, fluence, UV, and charged particles on the erosion of spacecraft materials. Miller et al. is one of the few experiments that has investigated these effects [23]. In their research, they exposed Kapton HN, FEP Teflon, and Polyethlene to a number of experiments in an isotropic RF plasma and hyperthermal beams of directed AO. In a series of tests, they controlled for factors like total AO exposure, arrival energy of AO, charged particles, and UV radiation. To block out UV radiation, the samples were shielded from direct exposure to the AO plasma by placing them in a series of different boxes 
that prevented a direct view factor of the UV to the samples while still leaving an opening for AO to indirectly scatter into the box. The charged species were blocked out using different variations of faraday cages around the samples. The varying fluence effect was achieved by running the tests for different lengths of time and looking at if the mass loss was linear with time or not. Lastly, the varying impact energy can only be achieved by testing in a facility that can accelerate the AO particles at the samples. The results for the erosion rate of each material were compared to Kapton H. This was an interesting decision by the researchers, as it is not known how Kapton $\mathrm{H}$ itself responds to each of these environments individually or synergistically. In any case, this test found that the effects vary from material to material. Polyethylene showed increased erosion with increasing $\mathrm{AO}$ effective fluence and decreased erosion with increasing arrival energy with respect to Kapton $\mathrm{H}$ erosion. It also showed a general trend of increased erosion with increasing charged particles. FEP Teflon showed an increase in erosion with increasing charged particles and a slight decrease in erosion with increasing $\mathrm{AO}$ effective fluence with respect to Kapton $\mathrm{H}$ erosion. The Teflon also showed a potential effect of UV intensity on the erosion, however, a previous test by the same authors did not show any such correlation. The Kapton HN showed a general trend of increasing erosion with increasing charged particles and a potential trend of increasing erosion with increasing fluence. In general it was clear from this research that different aspects of the environment interact differently with each material. All three materials were affected by charged particles, suggesting that there is a high likelihood that charged particles interact synergistically with AO. Between Miller et al. and Rutledge et al., there was no clear evidence that UV reacted synergistically with $\mathrm{AO}$ to cause different erosion rates than just the sum of the two environments independently [23, 34]. 


\section{$2.3 \quad$ Outgassing}

The vacuum environment results in an effect on materials known as outgassing that is a main focus of this research. Outgassing involves a material releasing trapped gases and volatiles over time through diffusion, desorption, or decomposition. This process is exacerbated when a material is placed in a vacuum and can result in many contamination problems. Diffusion involves trapped gas molecules working their way from within a material to the surface. Whether or not the molecule is able to escape the surface of the material is dependent on its thermal energy when it reaches the surface. Desorption is where a gas is released from the surface of a material if it can overcome the forces needed to escape. These forces can be chemical bonds or physical forces. Any material subjected to our atmosphere has some layers of gas molecules on its surfaces. Decomposition involves a material breaking down, undergoing chemical reactions, and otherwise changing in a way that results in the material itself outgassing. Most materials on Earth, regardless of their application, experience one or more of these forms of the outgassing process. In general, diffusion is the primary source of outgassing and it is a slow process compared to desorption as it does not usually take long for the few surface layers of gases to be adsorbed from a material. Decomposition is the least common form of outgassing. Outgassing products can include water vapor molecules along with other more volatile chemicals which can include solvents, catalysts, and incompletely polymerized polymers [14]. The volatiles are typically a result of imperfect manufacturing processes like improper curing or imperfect ratios of catalyst to resin.

There are multiple factors that can affect outgassing rates including activation energy, temperature, ambient pressure, time spent at that pressure, and otherwise processing history of the material. The activation energy relates to how strong the the bonds are that holds molecules on a material's surface and how much energy is 
required for a reaction to take place. Higher thermal energy results in more diffusion as it takes more thermal energy to move gases from the interior to the surface of a material. It also results in increased desorption as thermal energy is required for molecules to break away from the surface. Thermal energy also speeds up the decomposition process by increasing the available energy for reactions to take place that break down the material. The history of the material and how it was processed and/or manufactured can have a significant impact on the quantity of microscopic air molecules, as well as the type of trapped gases depending on what the material was exposed to during processing. The time spent at vacuum affects outgassing as it is not always a linear relationship for a given material or even a given mode of outgassing which is why the more detailed and complicated ASTM E595 test procedure was developed to look at outgassing properties over time. Table 2.2 compares the activation energy and time dependency of the three different modes of outgassing. The higher required activation energy for decomposition provides further evidence of decomposition contributing less to outgassing than desorption or diffusion.

Table 2.2: Activation Energies and Time Dependencies for Three Mechanisms of Outgassing [37].

\begin{tabular}{|l|l|l|}
\hline $\begin{array}{l}\text { Outgassing Mecha- } \\
\text { nism }\end{array}$ & $\begin{array}{l}\text { Activation Energy } \\
(\mathrm{kcal} / \mathrm{mole})\end{array}$ & Time Dependence \\
\hline Desorption & $1-10$ & $t^{1}-t^{2}$ \\
\hline Diffusion & $5-15$ & $t^{1 / 2}$ \\
\hline Decomposition & $20-80$ & $\mathrm{~N} / \mathrm{A}$ \\
\hline
\end{tabular}

For each mode of outgassing, it is possible to estimate the mass loss of a material over time. This outgassing rate is a function of the factors discussed above. For diffusion, the outgassing rate can be predicted by the equation [37] 


$$
\frac{\mathrm{d} m}{\mathrm{~d} t}=\frac{q_{0} e^{-E_{a} / R T}}{\sqrt{t}}
$$

Outgassing poses a very real set of potential contamination problems as outgassed contaminants have a tendency to collect and condense on other surfaces. When these surfaces are optics instruments or otherwise sensitive equipment the effects can be very bad. Because of this, it is necessary for contamination control engineers to understand how contaminants travel from their parent material to the surfaces they condense on. The one important parameter in this calculation is the view factor between the source and the surface of interest. The view factor is purely a geometric relationship and is given by the equation

$$
V F=\int \frac{\cos \theta * \cos \phi}{p i * r^{2}} \mathrm{~d} A
$$

where $V F$ is the view factor, $\theta$ is the angle between the normal vector of the source surface and the vector going from the source to the surface of interest, $\phi$ is the angle between the normal vector of the surface of interest and the vector going from the source to the surface of interest, $r$ is the distance between the two surfaces, and $A$ is the area of the outgassing source. Once the view factor is known, the mass arrival rate can be calculated using

$$
\phi=\sum_{s} V F_{s} \frac{\mathrm{d} m_{s}}{\mathrm{~d} t} \frac{1}{\rho_{s}}
$$

where $\phi$ is the arrival rate of mass, $d m / d t$ is the outgassing rate from eq. (2.2), $\rho$ is the density of the contaminant. The summation exists to include outgassing from all possible sources (hence the subscript $s$ ) as it is common for their to be multiple sources on an actual spacecraft. One thing that is interesting with outgassing is that a source does not actually need a direct line of sight to contaminate a surface. It is very possible 
for contaminants to outgas from the source to a new surface that has a line of sight to the surface of interest. It is possible for some of the contaminants that adsorbed on to the second surface to then re-outgas on to the surface of interest. This was observed on shuttle flights where sensitive instruments received contaminants when they had no line of sight to sources with such contaminants [16]. For applications where this may be a concern, it is important to understand what causes a contaminant to outgas from one surface to another and what causes it to adhere to a surface. In reality it is not a binary decision tree as to whether or not the contaminant adheres to a surface, but rather there is a probability of whether or not it condenses based on the temperature of the surface. Furthermore, if a contaminant does adhere to a surface, the amount of time that it will reside on the surface is finite and can be calculated using the equation

$$
\tau=\tau_{o} e^{E_{a} / R T}
$$

where $\tau$ is the residence time in $s, \tau_{o}$ is the typical oscillation period of the molecule on the surface in seconds (typically about $10^{1} 3 s$ ), $E_{a}$ is the activation energy mentioned earlier, $R$ is the ideal gas constant, and $T$ is the temperature of the surface. The temperature of the surface therefore plays a large role in determining the residence time of a molecule. As an example, water vapor on a surface at $100 \mathrm{~K}$ has a residence time of $10^{24}$ seconds, but on a $300 \mathrm{~K}$ surface the residence time drops down to just $\frac{1}{4}$ of a second.

So while all surfaces are equally likely to be contaminated by outgassed material, cold surfaces are much more likely to retain contaminants and cause them to condense. Unfortunately, surfaces that typically must be kept cold are usually instruments like optical lenses, mirrors, and sensors that are sensitive to contaminants. Even if the contaminants do not condense on a surface, the outgassed clouds of material can 
obscure the view between a sensor and the object it is trying to observe. This was shown to be the case with star trackers which have reduced tracking ability due to clouds of outgassed material [8]. Outgassing can also damage thermal surfaces and solar arrays. Thermal surfaces like MLI and radiators are carefully chosen and designed to have a specific absorptance and emissivity for the IR wavelengths in order to passively manage the temperature of the satellite in a delicate balance. Contaminants that condense on such surfaces can trap and/or scatter IR wavelengths, effectively degrading the performance of the MLI and radiators which will disrupt the balance of the spacecraft thermal management system. Thermal engineers know to design spacecraft for the end of life use case where passive thermal surfaces may be significantly degraded and active systems like heaters and louvers must make up for the decreased performance.

Thoughtful material design and placement can reduce the amount of outgassed material on exterior surfaces which will enhance the performance at end of life. Some design guidelines include the orientation of critical sensors to avoid direct view factors with any material known to outgas significantly. In addition, known outgassing sources can be placed to vent contaminants away from other surfaces. Most components and materials are also baked out in a vacuum chamber to remove as many contaminants as possible before the spacecraft is launched. Ground testing of units and materials plays an important role in the minimization of outgassing-related issues on orbit by providing information for engineers to reference when selecting materials.

The ASTM E595 standard was created so that different facilities could share data and results on different materials. Additionally, it ensures the tests at different facilities are conducted in the same manner. ASTM E595 defines two terms that are critical measures of outgassing of a material: TML and CVCM. TML stands for total mass loss; CVCM stands for collected volatile condensable materials. Both values are calculated as percentages of the original sample's mass and while the TML 
value is the total mass percentage of the sample that was outgassed during the test, the CVCM is only the percentage of the mass that condensed onto a collector disk that was initially clean. The standard also defines a method for determining the validity of the results of each test through the $1 / 10$ and $1 / 5$ tests. The document states that, in general, the results of a TML test can be considered valid if the standard deviation of the data is less than $1 / 10$ of the mean TML value for the test. Similarly, the CVCM results of a test are valid if the standard deviation of the data is less than $1 / 5$ of the mean CVCM value for the test. The goal of this test is to expose material samples to a vacuum less than $5 * 10^{-5}$ Torr and a temperature of $125^{\circ} \mathrm{C}$ for 24 hours. Meanwhile, the collector disks are maintained at $25^{\circ} \mathrm{C}$ with each disk having a direct view factor to a single sample. The higher sample temperature encourages outgassing while the cold collector temperature encourages contaminant condensation. The samples and collector disks are weighed before and after the test on a high precision scale in order to observe the small changes in mass. In general, materials are not recommended for spacecraft applications if they exhibit a TML of $>1.0 \%$ or a CVCM $>0.1 \%$ so the ASTM E595 standard allows for the pre-screening of materials for space applications. Materials that do not meet these guidelines are sometimes used, but extra precautions must be taken in order to ensure they do not cause any of the aforementioned problems.

\subsection{Outgassing with Atomic Oxygen and UV}

Atomic oxygen is not the only aspect of the space environment that can react synergistically with others, outgassing can as well. The two other environments that are of interest for this research are UV and AO. UV has been tested in ground experiments and on space with outgassing and has been shown to react with the effects of outgassing. 
First, UV radiation can actually increase the outgassing rate of a material. It does this by breaking bonds on a molecular level which speed up the decomposition of the material and make it easier for the disassociated molecules to be desorbed from the surface $[17,18]$. UV can also accelerate the deposition rate for a contaminant onto a target material and it can cause a contaminant to deposit on a surface that it otherwise wouldn't deposit on. Finally, UV has been shown to react with outgassed contaminants that have condensed onto surfaces by polymerizing and darkening the film of contamination [22].

When it comes to atomic oxygen and outgassing, there is not much literature on the subject. The work of Eli Gurnee in the Cal Poly Environments lab is in fact one of the few experiments on this topic and it is clear there is a need for additional testing [14]. It is very possible that AO will act similarly to UV with outgassing in the degradation of materials, as AO could make a material decompose faster while simultaneously freeing surface molecules for desorption. LEO atomic oxygen has the energy necessary to break bonds just as UV does. While ground based plasma ashers do not accelerate the $\mathrm{AO}$ relative to the samples, there are still a relatively high number of particles with a high energy. This is because the AO in the chamber has energy values that vary with a Maxwellian distribution and there are many more total particles as the tests are run at a relatively high pressure.

One study did show AO to interact with outgassed contaminants wherein a deposited contaminant layer on a quartz substrate was exposed to AO. The result was a stable surface layer of $\mathrm{SiO}_{2}$, which had a lower optical transmittance compared to the underlying quartz [13]. Another study tested O-rings to see how their TML and CVCM values differed when exposed to AO. The relevant results, shown in fig. 2.5, are the "As-received" TML and CVCM values as compared to the "with AO" TML and CVCM values where one of the O-rings is the S0383-70 and the other is the ELA-SA-401. 


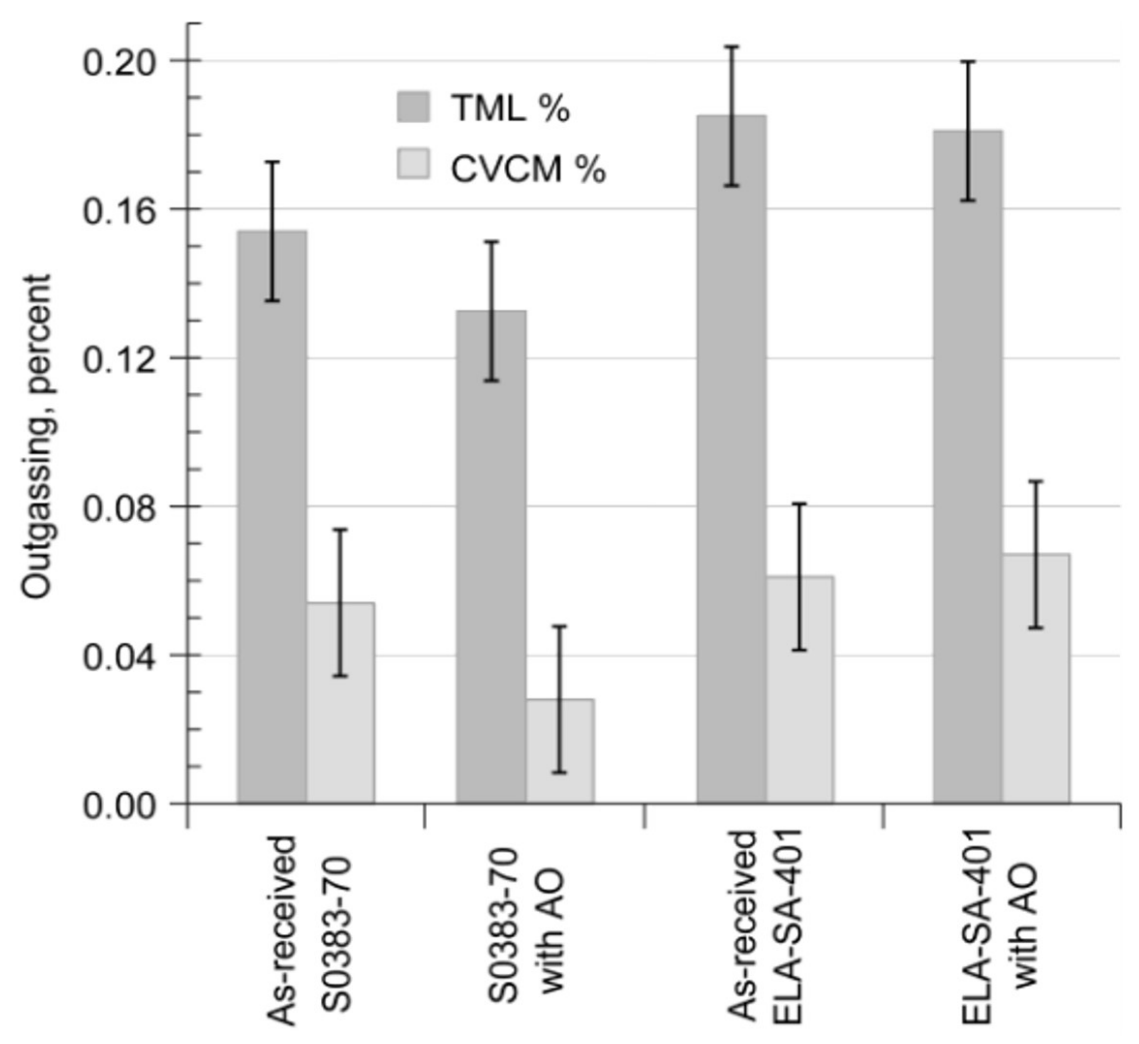

Figure 2.5: Effect of AO on the outgassing of S0383-70 and ELA-SA-401 O-rings [10].

The ELA-SA-401 set of O-rings did not show a significant difference between the AO exposed and control groups, but the S0383-70 AO exposed O-rings had a slight decrease in the average TML and CVCM compared to the control samples. The was overlap between the groups' error bars, yet the researchers still concluded there was a plausible trend of decreased outgassing due to AO. Generally these results would not be statistically significant, but the researchers observed a possible trend that they did not want to ignore and so they made a note of it. This approach is important to point out, as some of the possible trends in the results of this thesis had overlapping error bars, but the observed trend was in agreement with other statistically significant trends observed in similar materials. 
Chapter 3

EXPERIMENTAL APPARATUSES

\subsection{Apparatus (ELI)}

The Cal Poly Space Environments Lab is equipped with a chamber, referred to as ELI (Environmental mass Loss Investigation chamber), that is capable of running testing according to ASTM E595. The chamber was donated in 2013 by JPL in a non-working condition, and it had not taken data in many years. It was restored to be fully operational as part of Eli Gurnee's masters thesis and was then dismantled and used for other purposes until Fall of 2016 [14]. A big part of this thesis was getting the chamber and system working once again so that it could be used to determine material TML and CVCM values according to ASTM E595. A picture of the chamber and accompanying apparatuses, current at the time of this writing, is shown in fig. 3.1.

The main purpose of the chamber is to control the temperature and pressure that the samples and collector disks are exposed to. ASTM E595 is the materials testing standard that outlines the processes, procedures, and requirements to determine the outgassing properties of materials. The standard dictates that samples must be exposed to a vacuum at or below 5e-5 Torr for 24 hours while they are heated to $125 \pm 1^{\circ} \mathrm{C}$. That is what is necessary to determine the TML of the material. In order to determine the $\mathrm{CVCM}$, cold collector disks also need to be maintained at $25 \pm 1^{\circ} \mathrm{C}$ with a direct line of sight to one and only one material sample. The temperature and pressure requirements are somewhat arbitrary, but they stem from the concepts discussed in section 2.3 such as eqs. (2.2) and (2.5). These equations show how tem-

perature is related to the residence time of a contaminant and the outgassing rate of a material. The heated sample bar provides the thermal energy necessary for the 


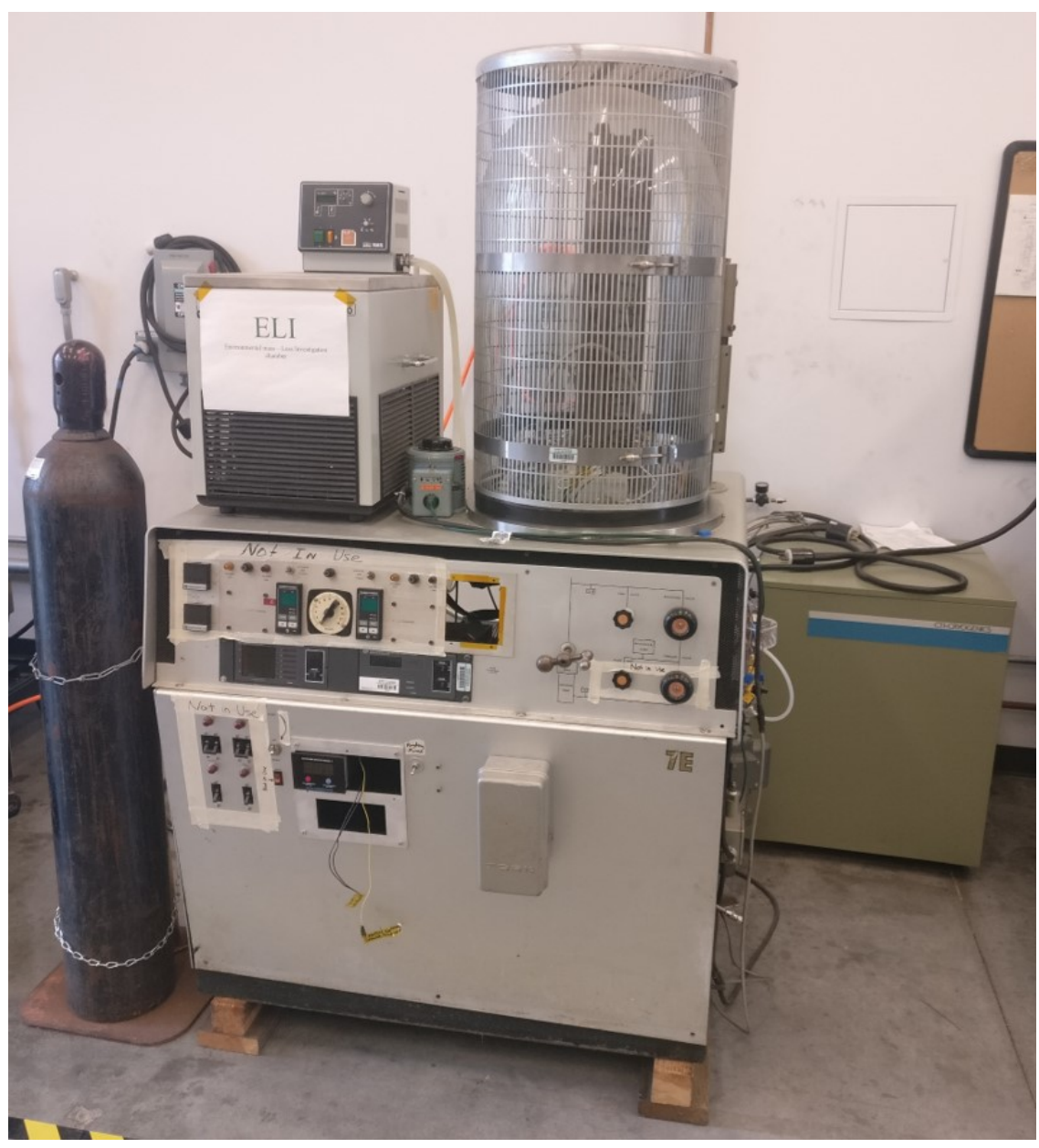

Figure 3.1: Outgassing testing chamber with water cooling unit and compressor.

samples to outgas, the cooled cold plate ensures a cold surface is available for contaminants to condense on with a long residence time, and the vacuum enables the gas molecules to spread out and separate from the surface of a material. The standard also specifies preparation, weighing, and other necessary procedures for the test, as well as how to calculate the outgassing metrics of interest. TML is calculated using the initial and final mass of the sample with the equation,

$$
T M L=\frac{S_{i}-S_{f}}{S_{i}} * 100 \%
$$

where $S_{i}$ is the initial mass of the sample in grams and $S_{f}$ is the final mass of 
the sample in grams after the 24 hour outgassing test is complete. Multiplying by 100 gets the number as a percentage. CVCM is calculated using the masses of the collector disks with the equation,

$$
C V C M=\frac{C_{f}-C_{i}}{S_{i}} * 100 \%
$$

where $C_{f}$ is the final mass of the collector disk in grams after it has been in the chamber collecting volatiles from the sample for 24 hours and $C_{i}$ is the initial mass in grams of the clean collector disk. An important note, TML is a measure of mass lost by a sample whereas CVCM is a measure of mass gained by the collector disk.

The ELI chamber is hooked up to a Welch 1397 roughing pump and a CTICryogenics Cryo-torr 8 cryogenic vacuum pump with either a CTI Cryogenics 1020R helium compressor or a CTI-Cryogenics 8200 helium compressor that provides compressed helium to the cold head of the cryo pump. The roughing pump allows the chamber to get down to as low as 30 mTorr. The cryo pump is used at pressures below 150 mTorr to pump the chamber down below 5e-5 Torr. The chamber interface is equipped with electrical feedthroughs for the sample bar heater, plumbing feedthroughs for water to cool the collector disk plate, type- T thermocouples to measure the temperature of the sample bar and cold plate, and a gas feedthrough to vent nitrogen into the chamber at the end of a test. Figure 3.2 shows a schematic of the chamber, its feedthroughs, pumps, and gauges. Here the convectron pressure gauge is represented by the CG bubble while the ion pressure gauge is shown by the IG bubble.

The sample containment bar and cold plate are exactly what is described in ASTM E595. The sample containment bar has two sides each with 12 sample containment chambers and cover plates. There is a $26 \Omega$ resistor welded along the length of each sample containment bar that heats up the bar to $125^{\circ} \mathrm{C}$. Currently only one side of 


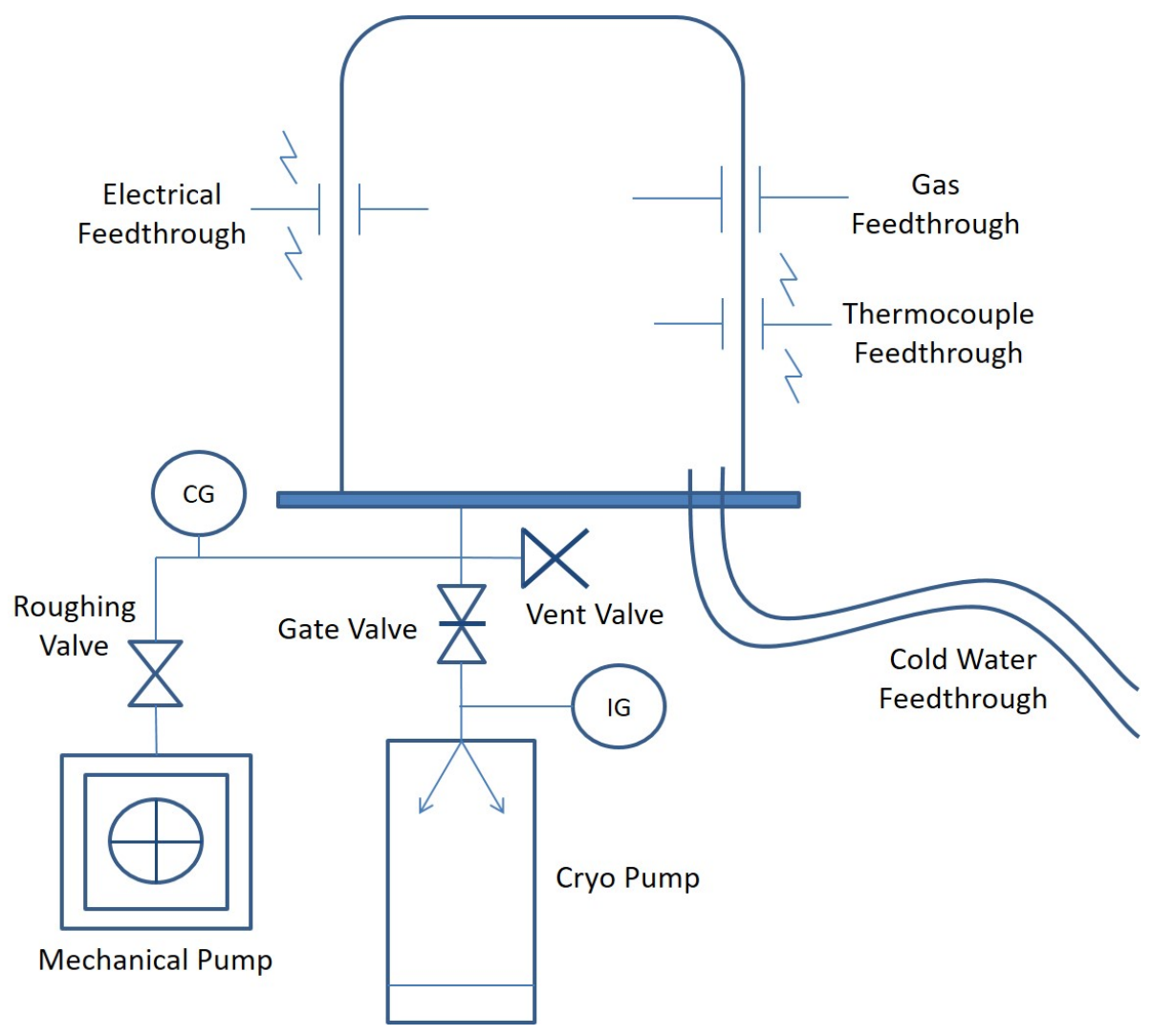

Figure 3.2: Full Schematic of ELI chamber.

the sample bar is operational so there are only 12 usable slots for samples. Each slot has a through hole that goes to the cold plate where the collector disks are maintained at $25^{\circ} \mathrm{C}$ in order to collect volatiles from each of the samples. Figure 3.3 shows a close up view of the test stand with the sample bar separated from the cold plate so that the UHV aluminum foil covered collector disks are exposed for easy access. Each collector disk is rested on two screw threads protruding from the cold plate, and it is pinned in place by a screw going through the cold plate with a spring on the other side so that the spring force keeps the collector disk pressed against the cold plate.

For testing, the cover plate (leaning on the right of fig. 3.3) is placed on the cold plate and then the sample bar is screwed back on, holding itself and the cover plate in place so that each collector disk is isolated from the other disks and samples. The three screws that hold the sample bar and cover plate in place are the only methods 


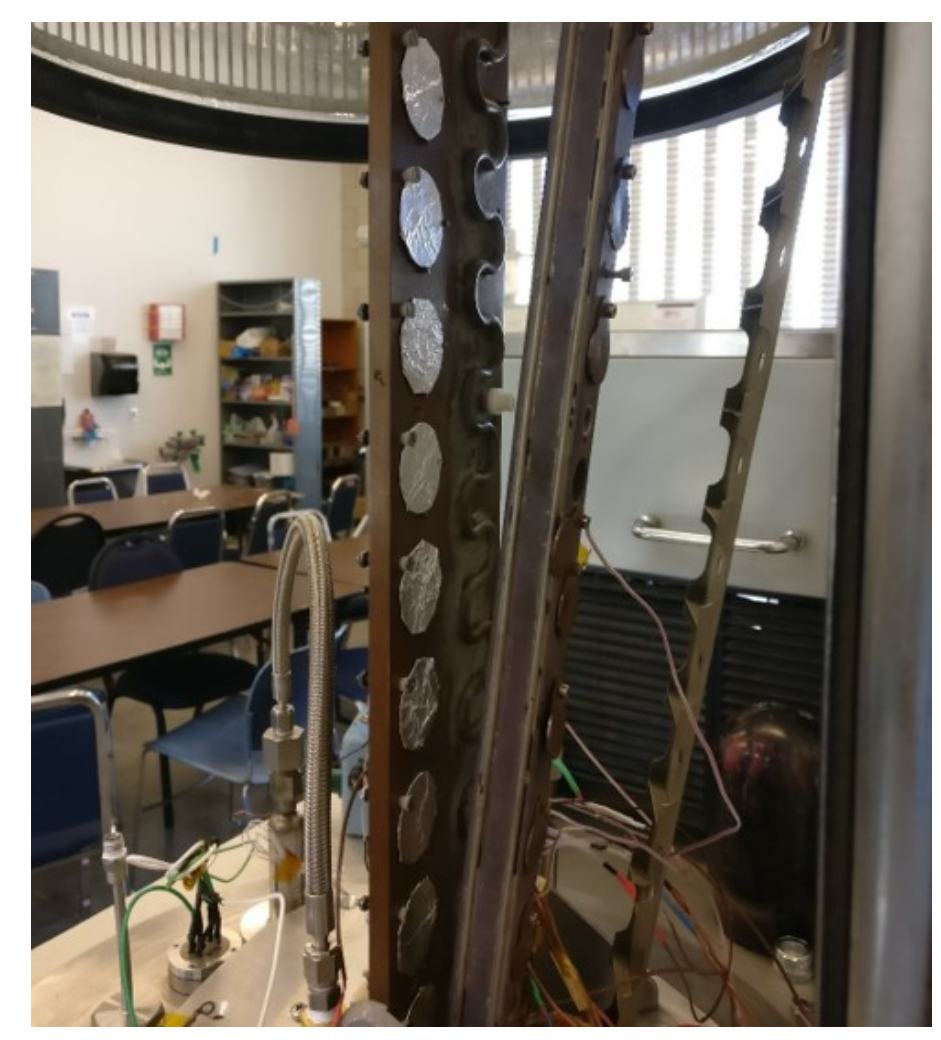

\section{Figure 3.3: ELI Chamber Test Stand Close-Up}

of heat to conduct between the hot sample bar and the cold plate which helps to limit heat transfer to primarily radiation. Finally each sample slot cover plate is screwed into place so each sample is isolated from the other samples.

Outside the chamber is the Brinkmann Lauda water cooling unit that runs the cold water through piping on the cold plate so it is maintained at $25^{\circ} \mathrm{C}$. There is also a Superior Electronics Powerstat Variac variable voltage power supply that is used to heat the sample bar to $125^{\circ} \mathrm{C}$. The thermocouple temperatures are read using an Omega HH506 thermometer. A Granville-Phillips 275 convectron gauge measures the pressure in the chamber from atmospheric down to $3 \mathrm{mTorr}$ while a Granville-Phillips 274 ion gauge measures pressures below that. The convectron gauge pressure is displayed on a Granville-Phillips 316 Vacuum Gauge Controller and the ion gauge pressure is shown on a Granville-Phillips 330 Ionization Gauge Controller. 
The cryo pump cold plate temperature is monitored with a Cryo Connections Model 2 Cryopump digital monitor.

\subsection{Refurbishment (ELI)}

At the start of this research, the ELI chamber had been dismantled for use in a different student project. This section details the work that went into repairing and replacing parts to bring the chamber back to working condition.

The piping for the water cooling unit had been disconnected and had significant leaks so a new hose as well as new connections were purchased from Swagelok. A braided stainless steel hose was chosen because the water pipe feedthroughs coming up from the bottom of the chamber are stainless steel, and the braided hose bent nicely into place to connect to the test stand pipes. The pipes coming off of the test stand are copper and they had been fitted with a Swagelok stainless steel tube fitting, the threads on which had been stripped and were another source of leaks. The tube fittings were replaced with new brass Swagelok tube fittings which were then connected to a stainless steel adapter that connects to the stainless steel braided hoses. One concern during this refitting was galvanic corrosion, which is a known issue when piping water between dissimilar metals. It is usually more of a concern when hard water is involved and the water cooling unit is intended to be used with deionized water. However, precautions were still necessary to ensure that corrosion would not build up as copper and stainless steel have significantly different corrosion potentials. The tube fitting was chosen to be brass because there is a lower potential for galvanic corrosion between copper to brass, or brass to stainless steel, as compared to the potential for corrosion between copper directly to stainless steel. Adding this intermediate brass connection was the best compromise, as the stainless steel feedthroughs must be connected to the copper pipe attached to the test stand and 
neither is easily replaceable. The water tank was also in need of a thorough cleaning after which the water cooling system was functioning properly.

Early on the heater power supply and sample bar resistor were another source of problems, as they would appear to work properly for hours before suddenly not working. This was very confusing because, in theory, it was not a complicated electrical set up. It consisted of just a power supply connected to feedthrough cables which attached to either end of the sample bar resistor inside the chamber. Some electrical troubleshooting eventually showed that one of the feedthrough cables would occasionally short and provide a path to chamber ground, while other times it would mysteriously work properly. These feedthrough cables, which come out of the front of the control panel, are spliced into larger coils of cables underneath the chamber and then eventually come out in one of the electrical feedthroughs in the chamber. It is important to note there are many unused controls on the control panel below the chamber. These include switches for a heater, diffusion pump, $N_{2}$, and a timer for the heater. Most of these cables are collected in the giant coils and some of them were fed through into the chamber so it was clear that previous effort was put into preserving those connections should someone want to use the control panel switches in the future. In order to determine where the short in one of those cables was, it would have been necessary to cut apart and potentially damage some of those connections so instead a separate electrical feedthrough was installed to hook up the heater power supply to the sample bar resistor directly.

One source of problems that prevents ELI from fully meeting the requirements of ASTM E595 is the way temperature is measured. ELI is equipped with eight t-type thermocouples that are connected to an electrical feedthrough so their temperatures can be read off using an Omega HH506 thermometer. The thermometer does not have data logging and can only take two inputs at once. ASTM E595 requires the sample bar be heated to $125 \pm 1^{\circ} \mathrm{C}$, which is such a tight temperature requirement that most 
other facilities use data logging thermometers with controllers that can adjust the voltage applied to the sample bar resistor so that temperature requirement is met. It was determined that the thermocouples on ELI are not of high enough accuracy to make an investment in an automated system worth while at this point. A Thermometrics representative revealed that t-type thermocouples have and accuracy of $\pm 1.0^{\circ} \mathrm{C}$ or $\pm 0.75 \%$ of the reading, whichever is greater. Furthermore, the accuracy of the HH506 thermometer for T-type thermocouples is $\pm 0.05 \%$ of the reading $+0.3^{\circ} \mathrm{C}$ for values between $-50^{\circ} \mathrm{C}$ and $1370^{\circ} \mathrm{C}$. This means that the total accuracy of a thermocouple connected to the thermometer is $\pm 1.36^{\circ} \mathrm{C}$ at a reading value of $125^{\circ} \mathrm{C}$. The error in the measurement is larger than the tolerance requirement, which means that even if the thermometer is showing the thermocouples at $125^{\circ} \mathrm{C}$, it is possible that they are not actually within the acceptable temperature range. The thermocouples ELI is equipped with are clearly not in perfect condition, however, as the eight thermocouples will show temperatures between $120-130^{\circ} \mathrm{C}$ for a surface roughly at $125^{\circ} \mathrm{C}$. Thermometrics suggested RTDs as an alternative to T-type thermocouple as they are generally more accurate (as accurate as $\pm 0.08^{\circ} \mathrm{C}$ ) and they can be easily surface mounted to something like the sample bar in ELI. These were not purchased due to cost restraints. Later conversations with Nataly Chen at JPL revealed that they use t-type thermocouples and they have not had any issues with accuracy.

One of the requirements of ASTM E595 is that the collector disks for the CVCM aspect be plated in chromium. The disks that the previous student used with his thesis are copper. It turns out it is not trivial to have thin copper disks plated in chromium as the plating process puts a tremendous amount of stress on the malleable copper. Another challenge with the original copper disks is that they weighed significantly more than the maximum weight allowed on the microbalance scale. Initially, a roll of thinner copper was purchased so that lightweight disks could be cut. The newly cut copper disks seemed to work well for one or two tests, however they could 
not be thoroughly cleaned, and after a few uses would start to produce erroneous results where they would lose mass instead of gaining it. After consulting with other facilities that perform this testing, it was determined that SSL uses chrome collectors while NASA JPL uses chrome disks that they cover with ultra-high vacuum (UHV) aluminum foil. UHV foil is essentially a very clean and contamination free aluminum foil that JPL has found to work very well for the ASTM E595 testing since it is small and light enough to be weighed on a full microbalance scale and it is disposable which eliminates the need to thoroughly clean and bake out the collector disks after each test. For those reasons, a roll of UHV foil was purchased and used for the CVCM aspect of the tests.

\subsection{Apparatus (MAX)}

The Minimum Atmospheric eXperimentation (MAX) chamber is capable of creating atomic oxygen to observe how materials react to the $\mathrm{AO}$ environment. It does this using an RF power supply that creates a plasma which in turn disassociates diatomic oxygen from air into atomic oxygen. There are two main types of systems capable of creating $\mathrm{AO}$ : those that accelerate the atomic oxygen relative to the samples, and those that do not. The latter are referred to as plasma ashers, which use the plasma to create the $\mathrm{AO}$ as MAX does. Plasma ashers have this name because they have been shown to be very successful at removing organic compounds, like carbon ash, from various surfaces, especially valuable artwork [33]. Plasma ashers typically run on air or pure $\mathrm{O}_{2}$ at pressures below 200 mTorr. This pressure is significantly higher than the pressure ELI is typically run at, however, the high pressure is necessary to supply enough oxygen molecules.

The power supply consists of an RF generator, a system controller, and a load and matching network which together provide power to an aluminum alloy electrode. 
The generator is a Seren R30MKII and can supply up to $300 \mathrm{~W}$ of power but it is only run at $125 \mathrm{~W}$ for the MAX chamber to create AO. Paired with the generator is the Seren AT3 matching network that matches the impedance load of the plasma generator. Without the matching network, the system load would reflect signals back through the coaxial cable and damage the RF generator. The controller is a Seren MC2, which is used to adjust the variable capacitors within the AT3 matchbox so that the reflected power can be minimized. The electrode is $15.25 \mathrm{~cm}$ in diameter and $0.9 \mathrm{~cm}$ thick and is surrounded by a dark space shield (DSS) that minimizes the secondary emissions from the electrode. The electrode is powered at a frequency of $13.6 \mathrm{MHz}$, which is designated for industrial plasma use. The other plate in the chamber is electrically grounded and is an aluminum square with $25.4 \mathrm{~cm}$ side lengths and spaced $7.62 \mathrm{~cm}$ from the RF electrode. As a result of the powered electrode and the ground plate, a capacitively coupled plasma $(\mathrm{CCP})$ is formed between the two plates. The DSS also serves to minimize power reflected back into the system and to make it easier for the plasma to be created between the two plates as opposed to between the electrode and one of the chamber walls. Both the ground plate and the DSS are grounded to chamber ground and power supply ground by copper ground straps. Figure 3.4 is an exploded view of the electrode and DSS assembly.

The ground plate has an aluminum cover plate with a \#8 mirror finish and has four precision machined holes that are $2.540 \pm 0.003 \mathrm{~cm}$ in diameter. These holes are equidistant from the center of the plate and ensure each sample that is clamped between the ground plate and the cover plate has an exposed area of $5.06 \pm 0.02 \mathrm{~cm}^{2}$. The cover plate is attached to the ground plate by 8 screws.

Atmospheric air is bled into the chamber to maintain a pressure of $175 \pm 10$ mTorr via a Swagelok tubefitting set in a hole in the bottom of the ground plate. The pipe attaches to a gas feedthrough in the chamber wall which is regulated by a black nupro valve. The high voltage electrode ionizes the nitrogen and oxygen, as well as 


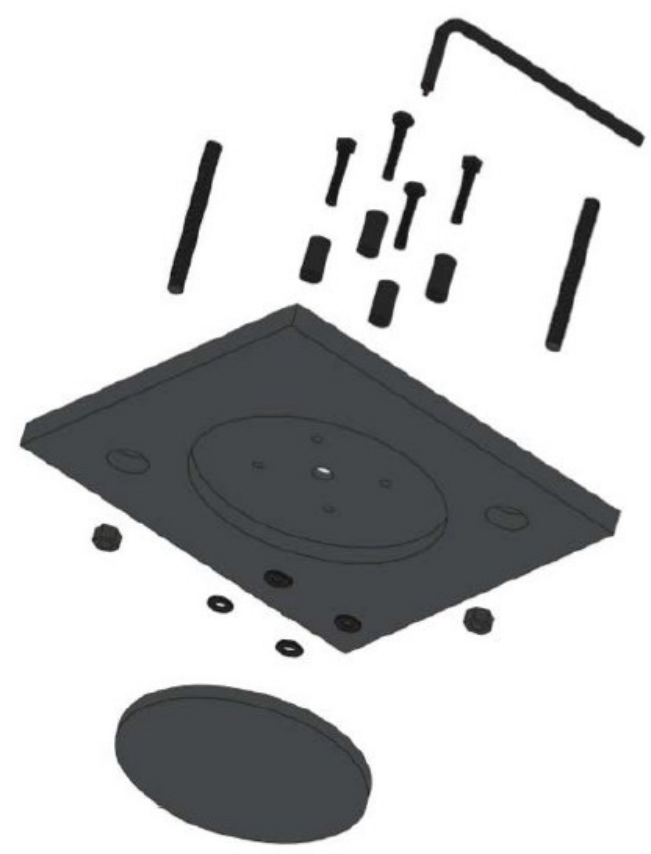

Figure 3.4: Top, powered plate in MAX comprised of circular electrode and square dark space shield. [12]

separates the $N_{2}$ and $O_{2}$ into atomic nitrogen and oxygen. The ionization energy lines correspond to pink and purple in the visible light spectrum. The ionized and atomic nitrogen have been shown in other studies to not significantly affect the results of material erosion rates due to $\mathrm{AO}$ and using atmospheric air is much safer than pure $\mathrm{O}_{2}$. MAX does not accelerate the $\mathrm{AO}$ so the energy of the atoms is about $0.01 \mathrm{eV}$.

A Welch 1397 roughing pump, the same type of pump used on ELI, is used to rough out the chamber and maintain the pressure requirement of $175 \pm 10$ mTorr. A Granville-Phillips 275 convectron gauge is used to measure pressure, and it is displayed on a Granville-Phillips 316 Vacuum Gauge Controller. Additionally, the chamber has the equipment necessary to achieve and monitor much lower pressures via a cryopump and ion gauges, but these are not used during $\mathrm{AO}$ creation. A set of K-type thermocouples are used to monitor the temperature of the ground plate during an experiment as it can get very hot and the temperature affects the outgassing 
of the material as well as the reaction rate of the materials with AO. Max Glicklin determined in his thesis that the suitable threshold for aborting a test would be if the ground plate temperature rose above $90^{\circ} \mathrm{C}[12]$.

MAX used to be equipped with a Hamamatsu L10706 deuterium lamp for simulating a portion of the VUV environment but it is in need of a replacement bulb and subsequent testing so it was not implemented in this research. A schematic of the chamber, pumps, gauges and feedthroughs is shown in fig. 3.5. Here the convectron pressure gauges are represented by the CG bubbles, while the ion pressure gauge is shown by the IG bubble.

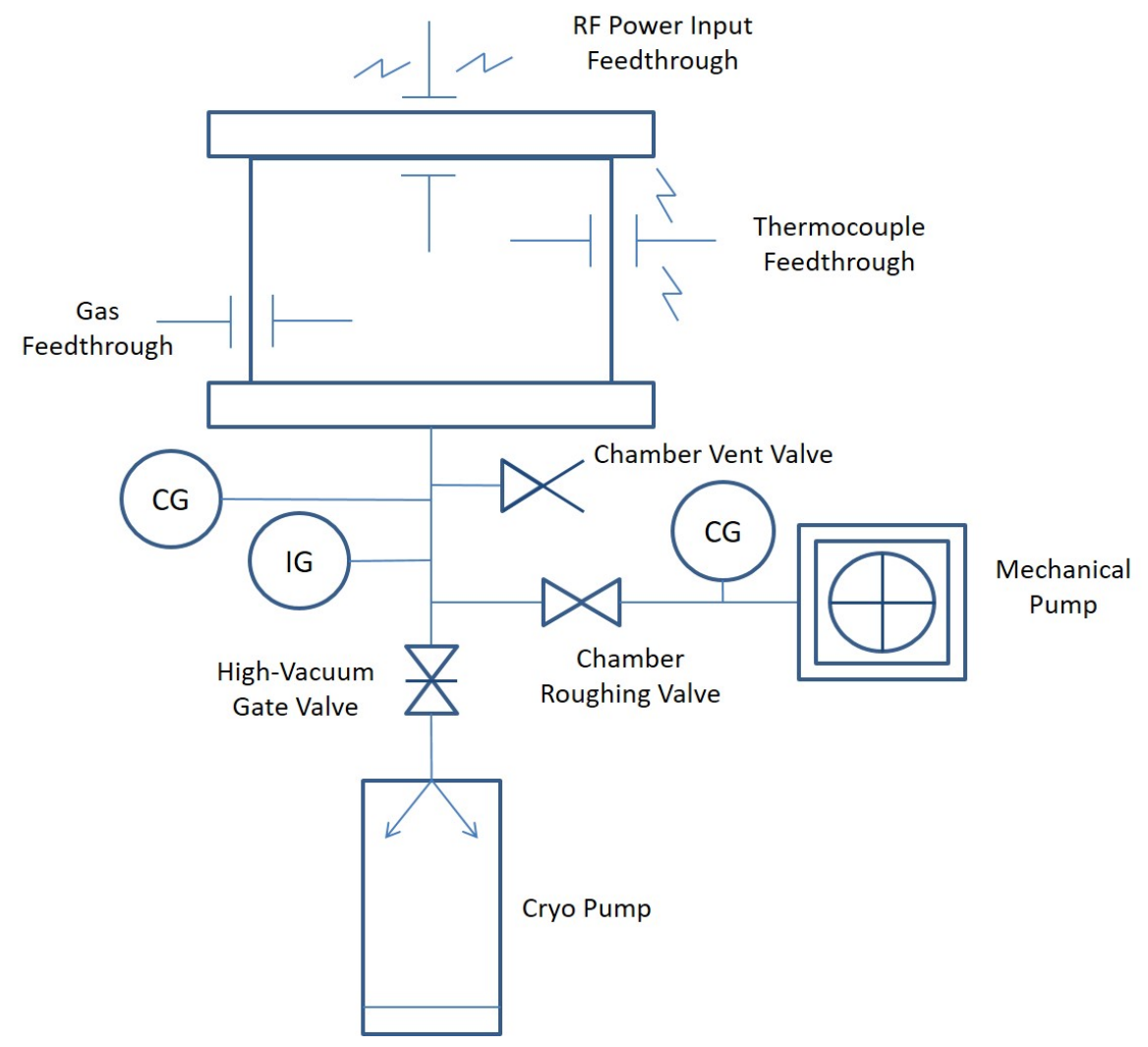

Figure 3.5: Full schematic of MAX chamber.

The resulting plasma created in MAX is an isotropic thermal plasma where the $\mathrm{O}_{2}$ component is made up of roughly $90 \% \mathrm{AO}$ and $10 \%$ ionized oxygen [14]. The AO atoms have an energy of $0.04-0.1 \mathrm{eV}$. As discussed in section 2.1, the AO environment 
experienced on orbit impacts spacecraft surfaces with a high concentration on the ram face at high velocities which results in the formation of pits and cones on many materials, especially polymers. This pit and cone formation is non-existent in MAX because the $\mathrm{AO}$ is isotropic with much lower velocity omnidirectionally. There are some AO chamber types capable of accelerating the $\mathrm{AO}$ relative to the material samples to produce the pits and cones effect, such as a laser detonation chamber, but these chamber types are generally significantly more expensive to build.

Aside from creating a plasma, the byproducts of MAX include VUV, high energy ions, and excited state neutrals which can all affect how a material responds to the environment and can cause a material to respond differently than it would to the on-orbit environment. A spacecraft in LEO will be exposed to each of these things, but at different concentrations and energy levels. Generally, plasma ashers generate relatively larger quantities of these secondary effects than are present in space. When it comes to UV, plasma ashers can have thousands of times more UV photons than the flux seen in space. This is not necessarily a issue as UV has not been shown to react synergistically with atomic oxygen. Ions and excited neutrals, on the other hand, have been shown to interact with AO synergistically. Plasma ashers do tend to create high numbers of these charged species leading to accelerated erosion of materials and surface interactions $[19,24,25]$.

Despite the byproducts of various types of AO chambers, ASTM E2089 does not define procedures or analysis methods to account for the side effects present in RF plasma testing. Instead the standard seeks to outline procedures that can be followed in a variety of differing test facilities. Equations (4.1) and (4.2) are defined in ASTM E2089 but these equations don't account for UV or charged particle exposure. Other specification documents like "Protocol for Atomic Oxygen Testing of Materials in Ground-Based Facilities" go a step further by categorizing AO chambers into three main types. A "Level 1" test facility is classified as being able to perform a screening 
test to determine in general if a material will have a significant reaction when exposed to AO. These facilities have charged particles and UV present in the test but they cannot be quantified. A "Level 2" test facility can mitigate some secondary effects by reducing or preventing charged particles or UV from reaching the samples. A facility in this category can also replicate the high particle energy of the AO found on orbit and can accelerate the AO relative to the samples. A "Level 3" test facility can isolate for, and intentionally combine, AO exposure with measurable amounts of charged particles and/or UV if desired in order to most closely replicate the conditions found on orbit in LEO [25]. According to these definitions, MAX is a "Level 1" facility capable of screening materials to get a baseline understanding of how they respond to $\mathrm{AO}$.

The point of this is to say that it is common for AO test facilities to not perfectly replicate the on-orbit environment. That does not mean that the results of such tests are invalid, it simply means that any conclusions drawn from data gathered using facilities like MAX should be more carefully questioned. In addition, one should be more hesitant to make generalizations from the data as the secondary effects cannot be controlled for or quantified. Having said that, in general, materials that react to $\mathrm{AO}$ plasmas in ground facilities also react to $\mathrm{AO}$ on orbit, and materials that are resistant to $\mathrm{AO}$ plasmas in ground facilities also are resistant to $\mathrm{AO}$ on orbit. Ultimately the mass loss that materials experience due to AO erosion in MAX is qualitatively similar to orbital erosion [14]. This means MAX meets the necessary requirements for testing in this research.

\subsection{Microbalance Scale}

For the purposes of this research a $\$ 20,000$ Mettler Toledo microbalance scale, model XS3DU, was purchased. It has a readability of 0.001 miligrams and a maximum 
capacity of $800 \mathrm{mg}$ at that readability. This scale was very critical to the research as the recommended sample size per ASTM E595 is 200 - 300 miligrams and most space rated materials are expected to have a CVCM of less than $0.1 \%$ which is less than $0.2-0.3$ miligrams. In addition, the test standard requires that a control disk be included in the tests with no sample in direct line of sight. This is to test to see if the sample bar was not cleaned properly, or if there is a significant amount of cross contamination between the samples and their respective collector disks. If the control disk gains more than 50 micrograms, that is an indicator that something is causing an issue. On the other hand, if any the collector disks lose 20 micrograms or more, that shows they were not cleaned properly prior to the test. Semi-microbalance scales do not have the precision necessary to make accurate measurements at this level.

One issue that the scale posed however was the maximum weight limit of 800 miligrams at the microgram level of precision. The scale can weigh masses up to 3.1 grams but the scale readability worsens by a factor of 10 to 0.01 miligrams which is undesirable. This was an issue because the collector disks that were used previously are 25 mil thick copper with a weight of about 6.3 grams each. This is another reason why the UHV foil was ultimately purchased as discussed in section 3.2.

The scale also has a harder time weighing dielectric samples that pick up static electricity as the measurement will drift considerably, sometimes for minutes on end. Mettler Toledo sells an anti-static unit that accompanies the scale for the purpose of solving this very problem. The model that accompanies the XS3DU microbalance is a small U-shaped electrode that a sample is passed through before it is weighed so the static electricity is neutralized. However, it costs approximately $\$ 2500$ and there are a number of significantly cheaper options that a Mettler representative suggested. Those include a hand held anti-static gun, a static master plutonium cartridge, or even laundry dryer sheets. The anti-static guns are those typically used to remove static electricity from an old-fashioned record player. These guns can be found on 
Amazon for less than $\$ 80$ and people have reportedly had success with them on many other objects besides just record players. The static master plutonium cartridges are small radioactive boxes that spew ions to neutralize the static buildup and they are placed inside the weighing area of the scale. Ultimately it was determined an anti-static gun would be the most effective tool for the cost so the Milty Zerostat 3 was purchased on Amazon for $\$ 73$. Later, after getting a tour of the SSL facility, it was observed that they use a 5th type of solution in the form of a Z-stat 6430 ionizer fan sold by Iso Stat Technology. These fans are available on eBay for a similar price range as the anti-static guns, however, it is unknown if they are a better solution. In any case, the anti-static gun did help to reduce static and ultimately significantly reduced the weighing time for certain sample types.

Another source of scale drift is regained water vapor mass that is apparent in samples recently removed from the temperature and vacuum of an ASTM E595 test. The test standard dictates that samples are to be weighed within one half hour of removal from the chamber and within 2 minutes of removal from the humidity controlled dessicator. This is due to the significant potential mass gain that a sample can experience due to regained water vapor. There is actually a separate section of ASTM E595 that outlines how to test and measure for water vapor regained during the first 24 hours of being removed from the chamber. This slow mass gain can be evident on the accurate microbalance scale which shows a gradual mass increase of about 1 microgram every few seconds.

It is difficult to isolate for all of the potential sources of scale drift, however if the sample has been allowed to cool to room temperature and it has been neutralized by some anti-static device, then it can be assumed that any scale drift left is due to water vapor being continuously re-absorbed and the samples should not be allowed to come to equilibrium in this case. They should be weighed as quickly and consistently as possible to minimize the regained mass. 


\subsection{Fourier Transform Infrared Spectrometer}

Fourier Transform Infrared (FTIR) spectroscopy was performed on three of the four silicone materials in order to see if there was a discernible change on a molecular level between the AO exposed samples and the initial untested materials. This was done using a JASCO FT/IR-4600 Fourier Transform Infrared Spectrometer. IR spectrometers in general use infrared radiation aimed at the sample while different sensors measure the amount of reflected and transmitted IR radiation at each wavelength. From there, absorption can be calculated if desired as reflectance, transmittance, and absorption must sum to $100 \%$. The IR radiation causes the bonds to vibrate in different ways including stretching vibrations, bending vibrations, and torsional vibrations. These responses correspond to peaks on a plot of transmittance over wavenumber. The size and shape of the peak, along with the location of the peak, are associated with particular molecules in a specific molecular vibration. This way, some of the chemical structure of a material can be determined from FTIR, and any changes in chemistry should show up as differences in peaks on the plot [15]. 
Chapter 4

EXPERIMENTAL TESTING

\subsection{Outgassing Testing}

Before beginning full synergistic testing, it was necessary to run some preliminary tests to see what the capabilities of the ELI chamber were, and to determine how it could be modified to best meet the requirements of ASTM E595. Ten tests were run on three different kapton materials to get an understanding of the pressures the chamber could reach and the temperature tolerances that were possible with the test stand and thermocouple set up. For these tests, it was determined that the ASTM E595 procedure could not be perfectly followed. The outgassing standard requires samples be weighed within 30 minutes of removal from the chamber with a full microbalance scale. At this point in the research there were still some issues with the microbalance scale drift that resulted in some samples taking up to 5 or more minutes before a weight could be recorded. This meant that at least 6 of the 12 samples were weighed after the 30 minute mark. In general, all weighing was completed within 1.5 hours from removing the samples from the chamber. In addition the sample bar could not be heated to $125 \pm 1^{\circ} \mathrm{C}$ within 1 hour. The tolerance was closer to $125 \pm 10^{\circ} \mathrm{C}$ and it took 1.5 hours to heat up to roughly $125^{\circ} \mathrm{C}$. The voltage on the Variac power supply could be increased, but that resulted in a larger temperature variation on the sample bar.

\subsection{Atomic Oxygen Testing}

The testing specifications for AO exposure are detailed in ASTM E2089 so that facilities around the world can generate comparable and easily reproducible data. 
Sections C.1 and C.4 outline the steps of this procedure as they apply to the MAX chamber in the Cal Poly Space Environments Lab. ASTM E2089 also defines two important calculations to make regarding each AO test: flux and fluence. As discussed earlier, the flux is the number of $\mathrm{AO}$ atoms that hit a material of a given area within a given time (usually one second). Fluence is the total number of AO atoms that hit a material of a given area over the course of the entire test. Fluence is calculated using,

$$
F=\frac{\Delta M}{A * \rho * E}
$$

where $F$ is the effective fluence in atoms $/ \mathrm{cm}^{2}, \Delta M$ is the change in mass in grams, $A$ is the exposed area of the sample in $\mathrm{cm}^{2}, \rho$ is the density of the material in $\mathrm{g} / \mathrm{cm}^{3}$, and $E$ is the reaction efficiency of the material in $\mathrm{cm}^{3} /$ atom. Flux can be calculated with the equation,

$$
f=\frac{\Delta M}{A * \rho * E * t}
$$

where $f$ is the effective flux in atoms $/ \mathrm{cm}^{2} / \mathrm{s}$. Flux is simply equal to the fluence divided by the total test time in seconds. It is important to note that the flux and fluence calculated with these equations are effective flux and fluence values. This means they are estimates for $\mathrm{AO}$ exposure if the material had been in LEO and experienced a similar mass loss. This is because the erosion rates used in the equation are one of four materials with a well known erosion rate when exposed to AO in LEO. Those materials include Kapton polymide (either H or HN), TFE fluorocarbon fluorinated ethylene propylene (FEP), low density polyethylene, and pyrolytic graphite [14]. The erosion rates for these materials remain roughly constant even though ground based tests erode the materials much faster than the actual on-orbit environment. For those reasons, the calculated flux and fluence are not necessarily representative of the num- 
ber of $\mathrm{AO}$ atoms the samples are actually exposed to in the chamber, but rather, the number of $\mathrm{AO}$ atoms necessary to result in the measured mass loss. The AO environment created in the chamber differs from the on-orbit environment in other ways as well. There are many interaction methods between AO plasma and a test material that include atoms with high thermal energy, high-energy ions, UV, neutrals, and isotropic $\mathrm{AO}$, which vary between different types of $\mathrm{AO}$ chambers as well as between different regions in LEO

\subsection{Synergistic Testing}

The experimental procedure for this research was important as small variations in temperature, pressure, and methods of pre-handling materials are known to have an effect on the differences in results between tests. Time was also a limiting factor as 9 of the 12 materials needed to be tested by a certain date to complete a student project contract with NASA JPL. This resulted in the procedure shown in fig. 4.1. In order to test as many samples as possible in the limited time frame, the minimum number of samples were tested: 6 samples of two materials resulting in 12 total samples per test which filled up the one working side of the test stand. Those 6 samples of each material were then split up into $3 \mathrm{AO}$ exposed samples and 3 control samples which is the minimum number of samples necessary to perform an ASTM E595 outgassing test. By utilizing all 12 of the sample containment chambers in order to test multiple materials at a time, the decision had to be made that a witness collection disk would not be included to check for cross contamination between samples and between collector disks. This was deemed acceptable because all of the tests in the system verification discussed above did not have an issue with the witness disk gaining or losing too much mass. This was an indication that the collector disk isolator plate and the cleaning procedures between tests properly ensured no cross contamination. 


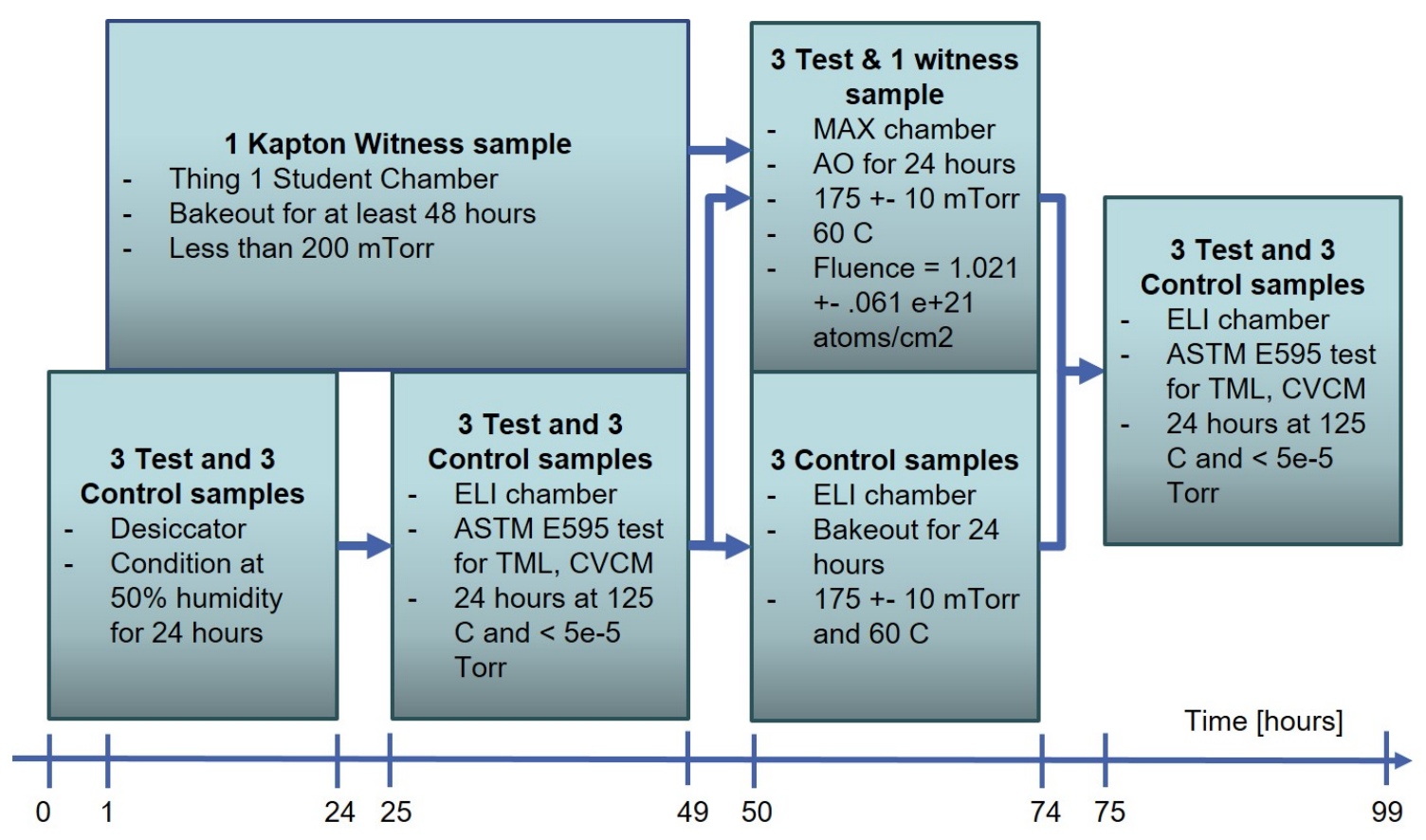

Figure 4.1: Synergistic Test Procedure

There were concerns in Eli Gurnee's work that some of the 12 sample containment chambers would heat up to a value outside of the $125 \pm 1^{\circ} \mathrm{C}$ requirement, while others met the requirement. Those fears were disregarded when it was discovered that the error in the thermocouple measurement was so large that such an observation could not be verified.

Once the JPL student project had terminated, there was some additional time to perform some follow up testing on a few of the materials. For these tests, only one material was tested at a time with 11 samples being split into $6 \mathrm{AO}$ exposed and 5 control samples. The 12th slot in the sample containment bar was left empty so that a witness collection disk could be included to check for any cross contamination. 


\section{Chapter 5}

\section{RESULTS}

\subsection{ELI Chamber Verification}

For the preliminary tests of the ELI chamber before synergistic testing began, the results showed the chamber was capable of maintaining a pressure just below $5 * 10^{-5}$ Torr (about $1-3 * 10^{-5}$ Torr). The temperature was not within spec as the thermocouples would read temperatures at $125 \pm 10^{\circ} \mathrm{C}$ which is well beyond the required tolerance of $\pm 1^{\circ} \mathrm{C}$. This issue was looked into, and after putting all of the thermocouples on the same spot on the sample bar, there was still the same variation in the readings. This suggested there is significant error inherent to the thermocouple and

reader system. A plot of this temperature variation over 6 of the tests is shown in fig. 5.1.

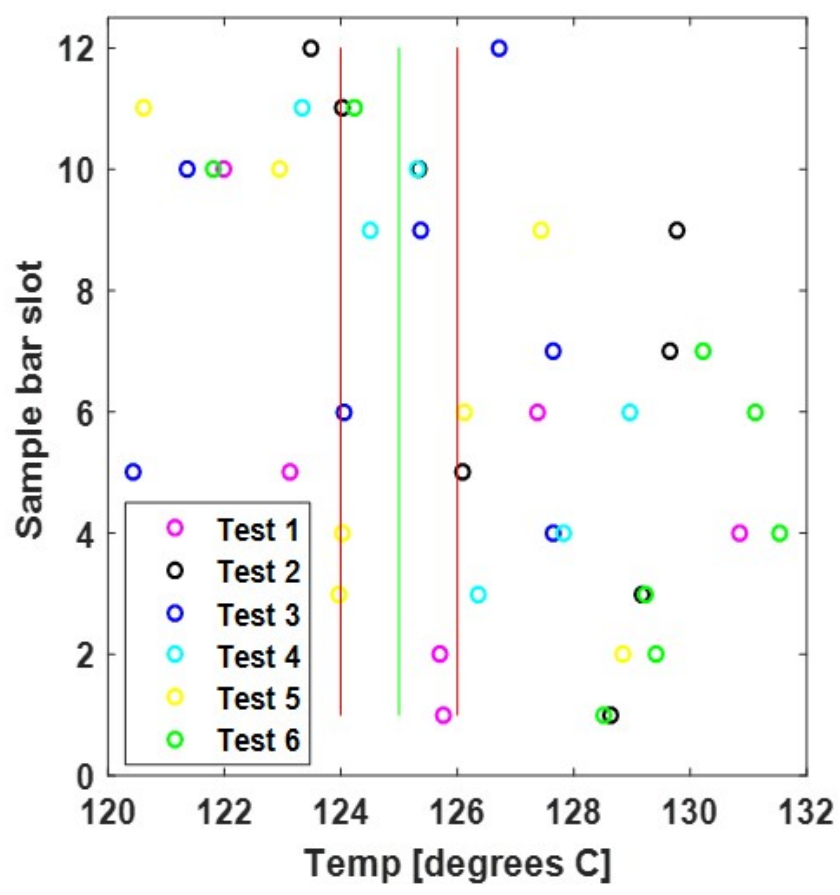

Figure 5.1: Sample Bar Temperature by Location 
To get this plot, thermocouples were assigned randomly for each test to a particular sample location and the recorded temperatures were averaged for each sample location over the test to produce one of the data points on the plot. Within each test and between tests there is clearly significant variation.

Table 5.1: Materials list with TML data. Asterisk denotes a similar material.

\begin{tabular}{|l|l|l|l|l|}
\hline Material & $\begin{array}{l}\text { Manufacturer } \\
\text { TML }\end{array}$ & $\begin{array}{l}\text { NASA } \\
\text { JPL TML }\end{array}$ & $\begin{array}{l}\text { Experimental } \\
\text { TML } \pm 1 \sigma\end{array}$ & $\begin{array}{l}\text { of } \\
\text { Samples }\end{array}$ \\
\hline NuSil R-2141 Sili- & $1.78 \%$ & - & $1.90 \pm 0.07 \%$ & 6,12 \\
\hline Tflex Silicone & $0.56 \%$ & $0.44 \%, 0.44 \%$ & $0.85 \pm 0.06 \%$ & 6 \\
\hline RTV 566 Silicone & $0.14 \%$ & $0.12 \%$ & $0.15 \pm 0.01 \%$ & 6,12 \\
\hline CV-2566 Silicone & $0.5 \%$ & $0.53 \%$ & $0.46 \pm 0.03 \%$ & 6 \\
\hline $\begin{array}{l}\text { M55 Graphite } \\
\text { Fiber/ Composite }\end{array}$ & 0.07 & - & $0.16 \pm 0.02 \%$ & 6 \\
\hline $\begin{array}{l}\text { EA 9360 Loctite } \\
\text { Epoxy }\end{array}$ & $1.73 \% *$ & $1.58 \%, 1.03 \%$ & $2.25 \pm 0.06 \%$ & 6 \\
\hline $\begin{array}{l}\text { Conathane EN-11 } \\
\text { Polyurethane }\end{array}$ & $0.38-0.45 \%$ & - & $0.45 \pm 0.02 \%$ & 6 \\
\hline
\end{tabular}

Table 5.1 contains a comparison of the TML values from different sources for all of the materials that were donated by JPL for this thesis. The "Experimental TML" column contains the experimentally determined TML as calculated from this research, and it includes \pm one standard deviation as obtained from the data of each test. The TML values here are from the first outgassing test, before any synergistic testing occurred. The "NASA JPL TML" column contains the TML value that the 
NASA JPL facility experimentally obtained when recently testing the material. The far right column lists the number of samples that were tested of each material for this research. The EA 9360, RTV 566, and the NuSil R-2141 materials were tested twice. However, as the EA 9360 was one of the first synergistic tests to be run, the procedure had been altered slightly by the time it was tested the second time. For this reason, only the values from the second test are listed for the EA 9360, while the other two retested materials list the averages of all of the tests (all 18 samples). The "Manufacturer TML" column contains the TML values that other research facilities obtained, either the values the manufacturer published in their material data sheets, or the values other NASA facilities obtained and published in the NASA Outgassing Database. For this column, if the exact material could not be obtained, then a similar material's TML value was used instead (denoted by an asterisk).

The first thing to note is that there can be some significant variation between facilities for the calculated TML values. For most of the materials, the experimental TML was within one or two standard deviations of the other facilities' calculated values. It is also interesting that the JPL facility found the Tflex TML values to be identical when they retested it, yet when they retested the EA 9360 they got a significantly different TML value than their first test. This is evidence of two potential sources of error; first, the material processing can have a significant effect on the results of an outgassing test. The EA 9360 is a Loctite Epoxy that must be mixed and cured and thus it is possible that variations in the mixture ratio or in the curing process can affect the outgassing properties of the material.

Second, there is a potential source of error inherent to the facility. TML values are very sensitive to many factors including the precise measurements taken on a full microbalance scale, humidity and temperature in the pre-processing and transition phases during the test, pressure in the chamber, and temperature on the sample bar. Variations in any of the above between tests can affect the results. Therefore, the 
Table 5.2: Materials list with CVCM data. Asterisk denotes a similar material.

\begin{tabular}{|l|l|l|l|}
\hline Material & $\begin{array}{l}\text { Manufacturer } \\
\text { CVCM }\end{array}$ & NASA CVCM & $\begin{array}{l}\text { Experimental } \\
\text { CVCM } \pm 1 \sigma\end{array}$ \\
\hline $\begin{array}{l}\text { NuSil R-2141 Sili- } \\
\text { cone }\end{array}$ & $0.49 \%$ & - & $0.61 \pm 0.12 \%$ \\
\hline Tflex Silicone & $0.10 \%$ & $0.12 \%, 0.12 \%$ & $0.39 \pm 0.04 \%$ \\
\hline RTV 566 Silicone & $0.02 \%$ & $0.00 \%$ & $0.04 \pm 0.01 \%$ \\
\hline CV-2566 Silicone & $0.03-0.10 \%$ & $0.03 \%$ & $0.09 \pm 0.01 \%$ \\
\hline $\begin{array}{l}\text { M55 Graphite Fiber/ } \\
\text { Composite }\end{array}$ & 0.00 & - & $0.05 \pm 0.01 \%$ \\
\hline $\begin{array}{l}\text { EA 9360 Loctite } \\
\text { Epoxy }\end{array}$ & $0.01 \% *$ & $0.00 \%, 0.00 \%$ & $0.01 \pm 0.00 \%$ \\
\hline $\begin{array}{l}\text { Conathane EN-11 } \\
\text { Polyurethane }\end{array}$ & $0.01-0.03 \%$ & - & $0.02 \pm 0.00 \%$ \\
\hline
\end{tabular}

notion that TML values calculated by different facilities should match is not always a reasonable expectation. One of the materials tested for this thesis was a graphite fiber composite that did not exhibit a TML near the value that was found in the NASA outgassing database. The discrepancy could be due to a number of factors. First, that material was donated by JPL as a part of a student project because it was beyond its shelf life and they wanted to see if the outgassing values would be different. It is possible the discrepancy in the TML value can be explained by how old the material is. It is also possible that the pre-processing of the material influenced the amount of trapped gasses within it. The material was donated to Cal Poly in an uncured state and was stored in a freezer. A couple of weeks before the material was 
laid up for this research, the freezer began to not function properly and it could not maintain the cold temperatures that are typically required. This unexpected heating, or the curing process, could have introduced more microscopic contaminants than usual to cause the TML value to be much higher.

Table 5.2 is similar to table 5.1, except instead of the TML values, it lists the CVCM values of the same materials from the same tests. As was true with the TML values, the experimental CVCM calculated in this research for the Tflex was larger than that found at other facilities which suggests it may be worth retesting to see if that test result was an anomaly. Aside from that, all of the other values fall within the range of acceptability.

\subsection{MAX Chamber Verification}

In general there was not much work necessary in order to verify that MAX was a system capable of exposing samples to AO according to ASTM E2089. The chamber has been tested and verified in the previous work of other students, and it still runs consistently $[14,12]$. It was necessary to run some initial tests to determine if the samples experience a uniform AO exposure everywhere on the ground plate. This was necessary because of differences between the sample holders between ELI and MAX. On the one hand, ELI has sample chambers that are approximately $12.3 \mathrm{~mm}$ deep and $16 \mathrm{~mm}$ in diameter, whereas the sample slots on MAX are $25.4 \mathrm{~mm}$ in diameter and designed for thin samples. It is not really possible to get a thin square sample that is larger than $25.4 \mathrm{~mm}$ on a side to fit in the smaller space in the ELI sample slots, so a compromise had to be made where the samples would be cut to fit inside the ELI sample chambers and then they would be placed on top of the cover plate in MAX. This was really the only way the samples could be exposed to AO due to the configuration of MAX, and as a result, there was no way to precisely guarantee 
the samples would be exposed to the same amount of AO. This was observed to be a significant issue on one of the preliminary tests where long strips of coated kapton materials were tested. The strips had to be long enough to meet the minimum sample mass requirement of $200 \mathrm{mg}$ set by ASTM E595, and this was not a problem for the ELI sample chambers because the strips could be rolled and folded to fit in the small volume. In MAX however, the strips had to be laid out and taped down to the cover plate. Figure 5.2 shows the materials before and after they were exposed to AO.

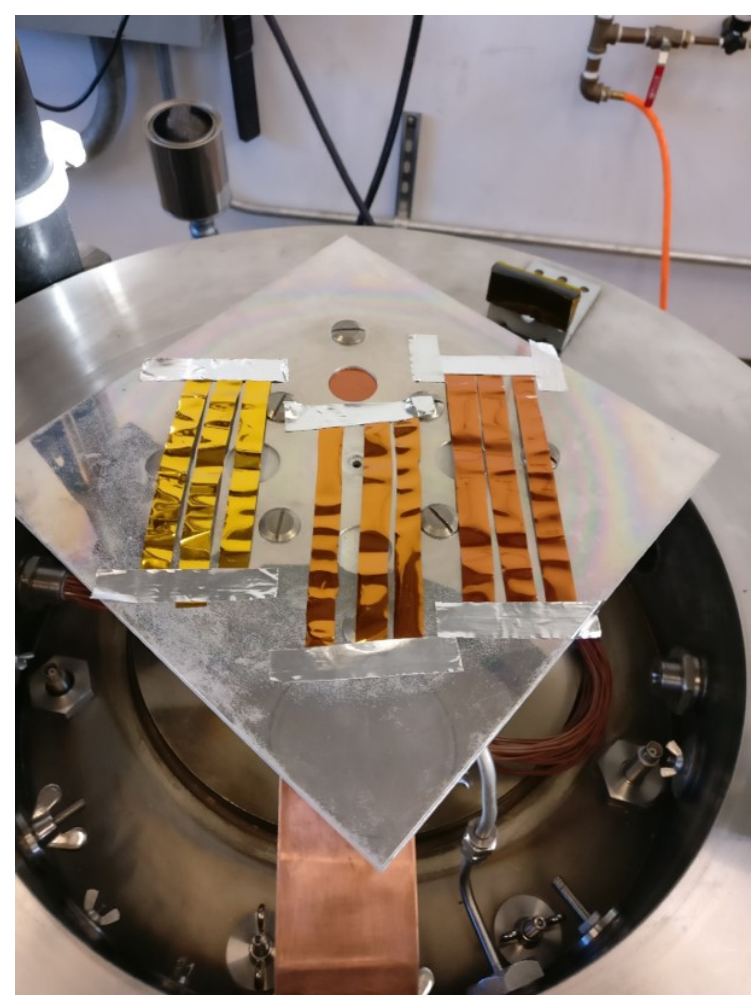

(a) Three Kapton materials before AO exposure

Figure 5.2: Kapton tape, 2 mil Kapton, 3 mil Kapton (left to right) before and after $\mathrm{AO}$ exposure with a Kapton $\mathrm{HN}$ witness sample in the top center

The set of three samples on the far left (Kapton tape) had the thinnest kapton layer of all of the materials at only 1.0 mil thick. As can be seen in fig. $5.2 \mathrm{~b}$, the sample on the right of that set lost all of the Kapton during the test and all that remained was the aluminum, while the sample on the left of that set still had a 
significant amount of Kapton at the end of the test. The sample in the center of that set shows a clear outline of the shape of the electrode that provides the RF power to create the AO plasma. This test clearly indicated that there is a significant gradient in $\mathrm{AO}$ exposure at the edge of the projection of the $\mathrm{RF}$ electrode on the ground plate.

It was difficult to come up with a good method that would ensure long sample strips all experienced the same amount of $\mathrm{AO}$ exposure, so the results from the synergistic testing of those sample types were not included in the findings of this research. Furthermore, this test showed the importance of ensuring all samples of a material are placed an equal distance away from the center of the ground plate as best as possible. This is normally accomplished by placing them under the cover plate within one of the 3 remaining sample slots, but since that was not possible for the synergistic testing, the samples were often placed in those locations once the cover plate had been screwed in place as is shown in fig. 5.3.

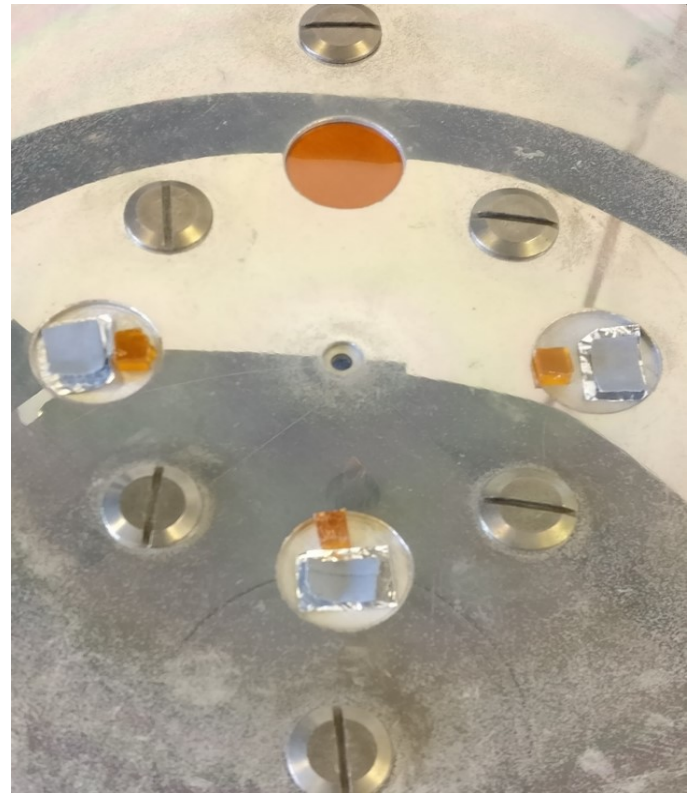

(a) Tflex and EN-11 before AO exposure

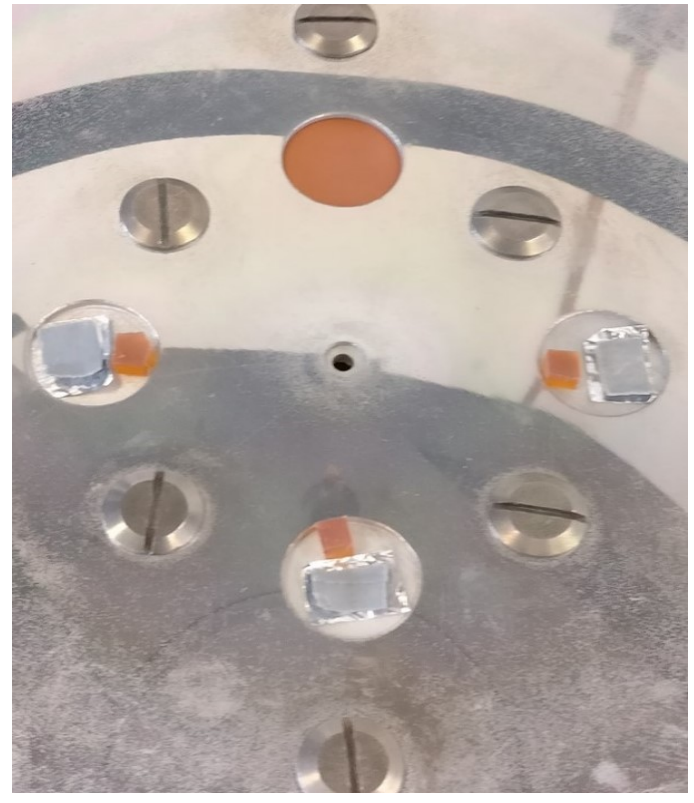

(b) Tflex and EN-11 after AO exposure

Figure 5.3: Tflex silicone thermal gap filler (grey rectangles) and EN-11 polyurethane (orange cubes) before and after AO exposure with a Kapton HN witness sample in the top center. 


\subsection{Synergistic Testing}

The bar charts in the following figures are the results of the synergistic testing. Each plot of four bars is for one material, with the TML values as the turquoise colored bars and the CVCM values as the magenta colored bars. The subscripts on the bars, along with the plot label, describe what tests from fig. 4.1 are being compared, as well as what sample groups are being compared. An AO exposed vs control label indicates the TML and CVCM values after the final outgassing test for the samples exposed to AO as compared to the control samples. An I1 and I2 label denotes the values calculated for the initial outgassing test, but specifically for materials that were retested and thus have multiple data sets for the initial outgassing values. Lastly, an I vs C labeled plot shows the difference in outgassing values for all samples in the initial outgassing test compared to the control samples in the final outgassing test. On each bar there are two error bars, a black and a green error bar. The purpose of this is to compare the standard deviations in the data to the acceptable $1 / 10$ and $1 / 5$ tests discussed in section 2.3. As is outlined in ASTM E595, the results of an outgassing test can be determined acceptable if the standard deviation of the TML values is less than $1 / 10$ of the average TML value, and if the standard deviation of the CVCM values is less than $1 / 5$ of the average CVCM value. In the bar graphs the black error bar is \pm 1 standard deviation in the data while the green error bar is $\pm 1 / 10$ of the average TML value or $\pm 1 / 5$ of the average CVCM value. If the green error bar encompasses the black error bar, then the data had an acceptable amount of variation. If the black error bar extends beyond the green error bar, then it did not pass the $1 / 10$ or $1 / 5$ test. In this case, the results should be analyzed skeptically, and the material should be retested if possible.

The bar charts in fig. 5.4 show the AO exposed vs control groups for the EA 9360 which was retested. Figure 5.4a shows the results from the first time the material was 
tested, while fig. 5.4b shows the results from the second test of the material. There are a number of things that are worth noting about the differences between the two tests. In the first test, the minimum number of samples were used ( $3 \mathrm{AO}$ exposed and 3 control) in order to get TML and CVCM values. Additionally, while testing the material for the first time, the cryo pump malfunctioned during the middle of the final outgassing test. Shortly thereafter, the cryo pump was replaced with a different cryo pump that was not being used on a different chamber. Once the new cryo was in place, the outgassing test continued and was finished. However, in the down time of about one day, the samples were brought back up to atmospheric temperature and pressure, which in itself invalidated the test results. The test was only finished for the sake of comparing the data to a later test. In the second test, 11 samples were used (6 AO exposed and 5 control) and the test went smoothly from start to finish.

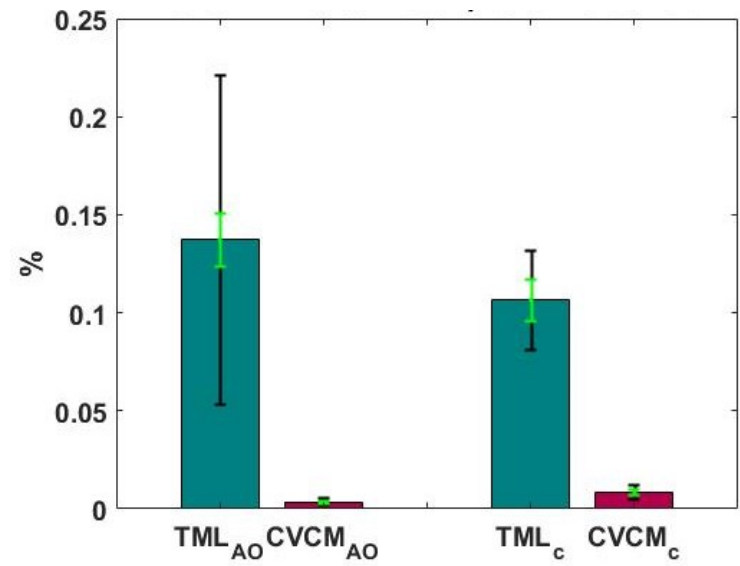

(a) EA 9360 AO Exposed vs Control

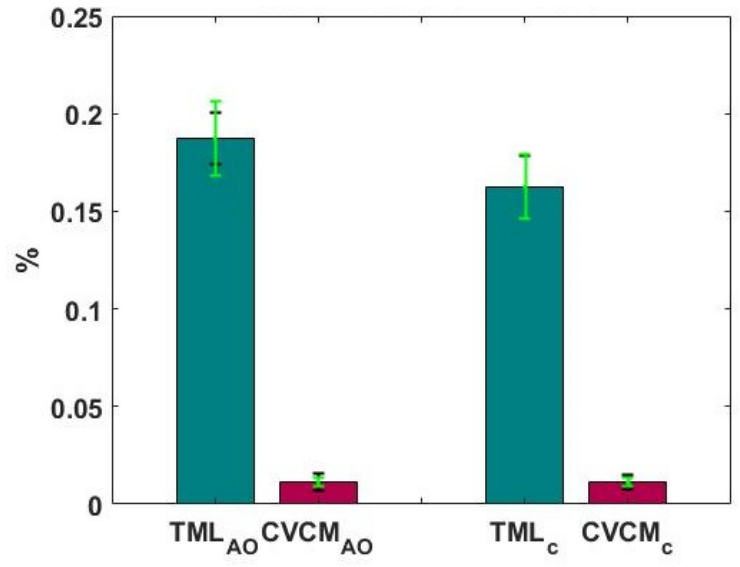

(b) Retest of EA 9360 AO Exposed vs Control

\section{Figure 5.4: EA 9360 Samples TML and CVCM Outgassing Results}

The four plots in fig. 5.5 show the results from the final outgassing test of four different silicone materials for the AO exposed samples compared to the control samples. The CV-2566 and the RTV 566 are both silicone rubbers, the Tflex is a reinforced silicone thermal gap filler, and the NuSil R-2141 is a two part silicone adhesive. All of these materials except the CV-2566 showed a lower TML for the samples exposed 
to $\mathrm{AO}$ and two of them showed a lower CVCM. The Tflex did have too large of error bars, as did some of the other materials before they were retested with more samples. Despite the large variation in the data for that material, there was still no overlap in the error bars so the data was included in the results. The CV-2566 could possibly have had a lower TML for the AO exposed samples as the mean value for that group was lower, but there was overlap in the error bars so the change can't be confirmed.

The effect of AO on TML and CVCM for the other materials varied based on the material. For AO exposed samples, the EN-11 polyurethane showed a lower TML, the M55 composite showed a higher TML, and the EA 9360 Loctite Epoxy (shown in fig. 5.4) had a slight decrease in the average TML. For the latter, there was overlap in the error bars.

The three plots in fig. 5.6 compare the results of the first outgassing test of the procedure in fig. 4.1. If the samples came from the same batch of material, one would expect the initial TML and CVCM values to be roughly identical, however, the results show this was not necessarily the case as there was some significant variation between the two separate tests of the EA 9360. Having said that, this difference cannot be given too much weight due the the issues in the first time the EA 9360 was tested.

The last set of graphs in fig. 5.7 show how the outgassing values significantly decreased for all of the silicone materials between the initial and final outgassing tests. This held true for all of the materials tested and thus is why the control samples' TML and CVCM values after the second test should not be compared to other facilities calculated values. There is a clear mechanism here causing the samples to outgas much less in the second test.

Additional pictures of samples, including before and after AO exposure pictures, can be found in chapter B. Some of the materials displayed a noticeable visual change after different parts of the test procedure. Multiple materials exhibited a physical 


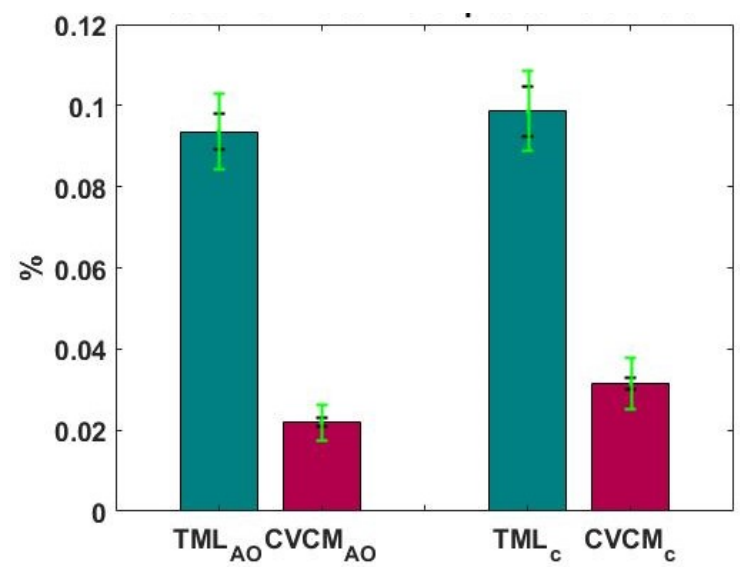

(a) CV-2566 AO Exposed vs Control

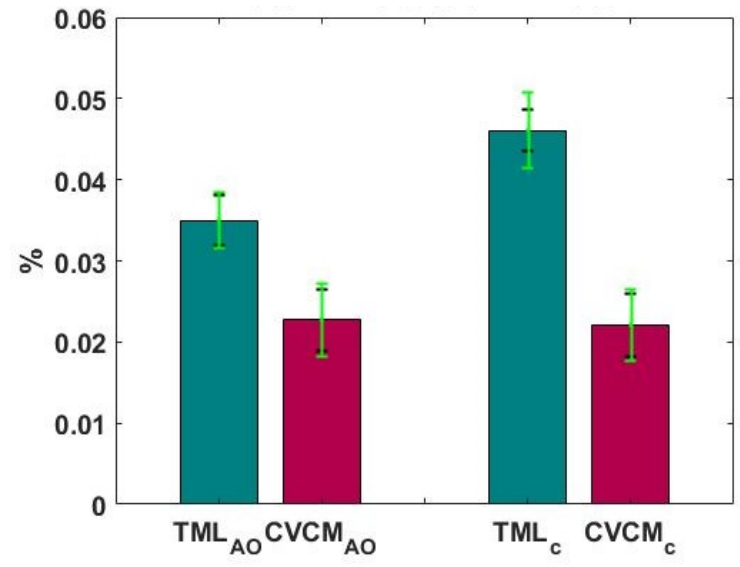

(c) RTV 566 AO Exposed vs Control

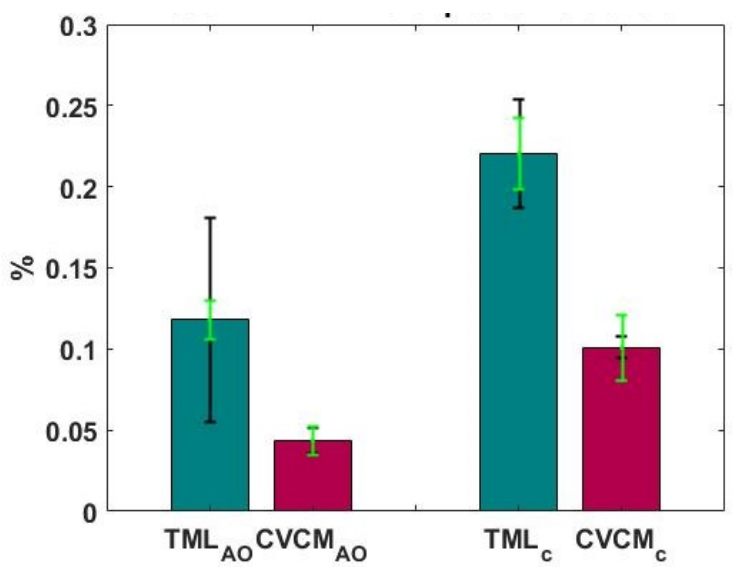

(b) Tflex AO Exposed vs Control

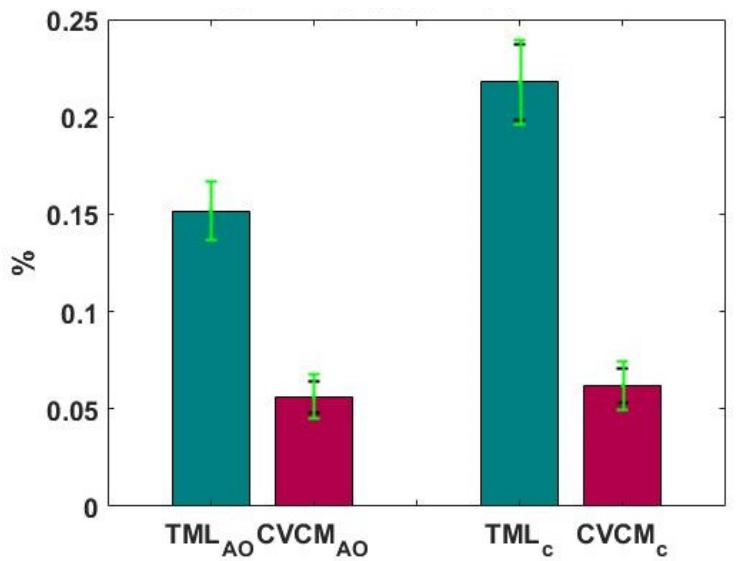

(d) NuSil R-2141 AO Exposed vs Control

Figure 5.5: Silicone Materials TML and CVCM Outgassing Results for AO Exposed Samples in the Final Test Compared to Control Samples in the Final Test

change from the $\mathrm{AO}$ exposure, which was expected. Some of these included the discoloration and reduction in reflectivity of the Kapton materials. The $\mathrm{SiO}_{2}$ coated kapton had a significant visual change in the surface properties which was surprising because the coating is supposed to resist degradation due to AO. The Tflex became very brittle as a result of the $\mathrm{AO}$ exposure and some of the samples even broke into pieces from very gentle handling as seen in fig. B.5. Both the NuSil R-2141 silicone adhesive and the EN-11 polyurethane darkened and became more opaque. Some of these changes were expected, and it was not surprising that the materials exhibited 


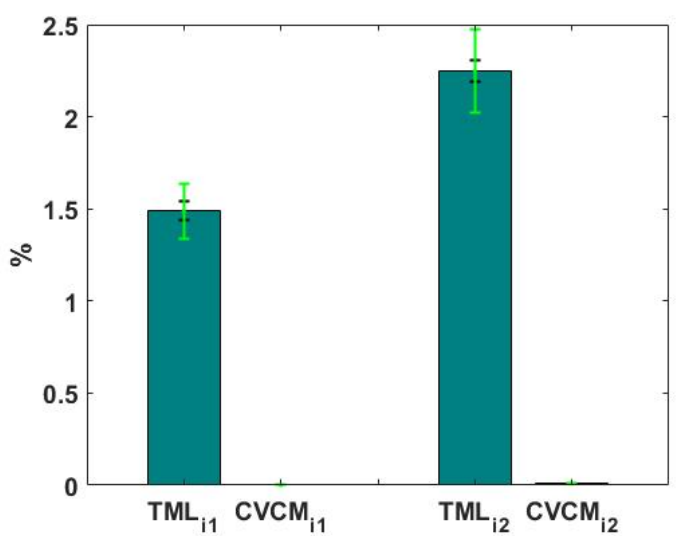

(a) EA 9360 First \& Second Initial Tests

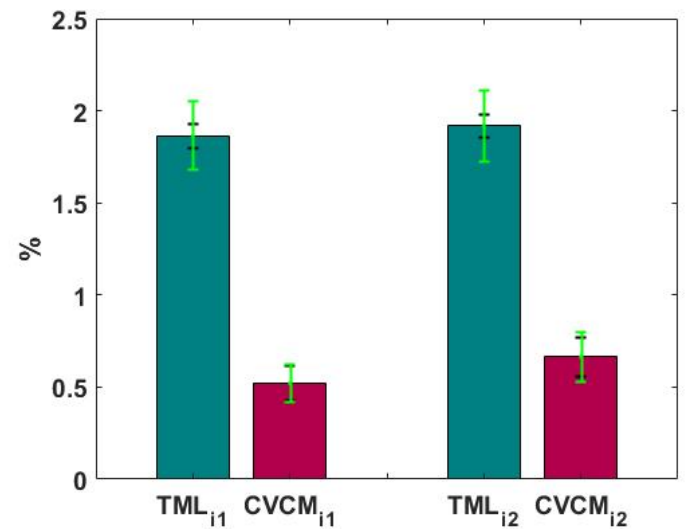

(b) NuSil R-2141 First \& Second Initial Tests

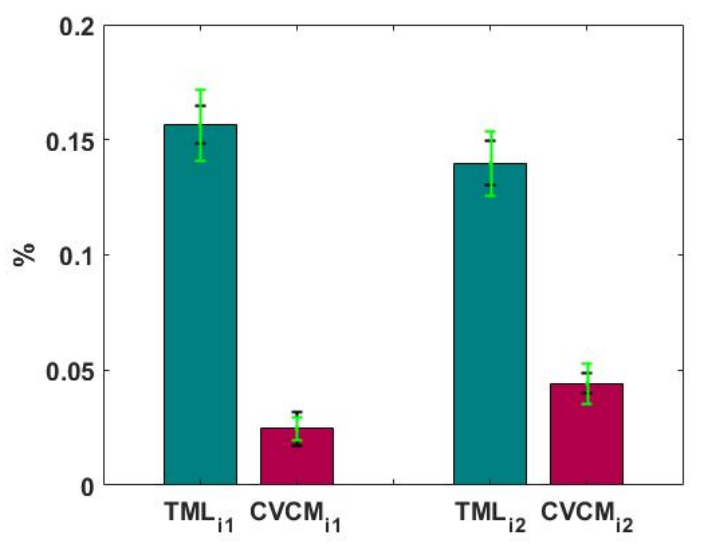

(c) RTV 566 First \& Second Initial Tests

Figure 5.6: Retested Samples TML and CVCM Outgassing Results for All Samples in the First Initial Test Compared to All Samples in the Second Initial Test

visual degradation from the $\mathrm{AO}$ exposure. What was not expected was that some of the materials would have significant changes as a result of just outgassing. The EN-11 polyurethane and the EA 9360 Loctite adhesive both underwent noticeable color changes solely as a result of being in a vacuum. The color changes for these materials are shown in figs. B.4 and B.8. Other materials like the RTV 566 and CV-2566 silicone rubbers did not show any noticeable changes.

For each of the AO tests, the included kapton witness sample was weighed before and after $\mathrm{AO}$ exposure so that the effective flux and fluence of the test could be 


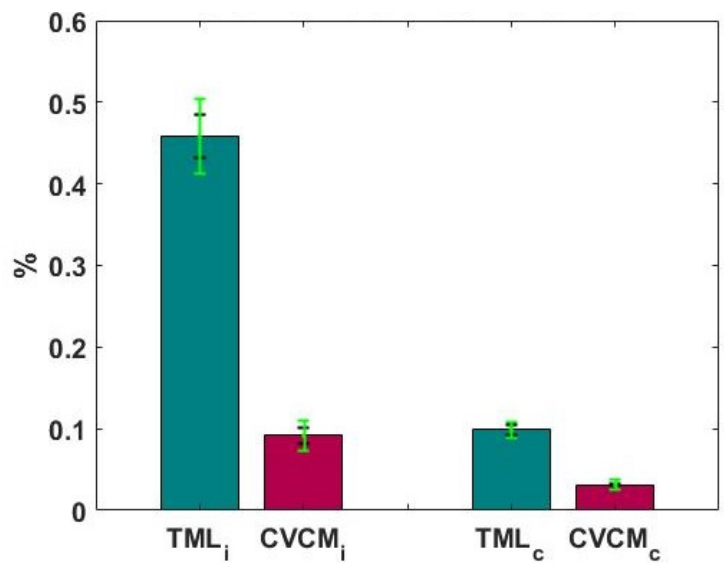

(a) CV-2566 Initial vs Control

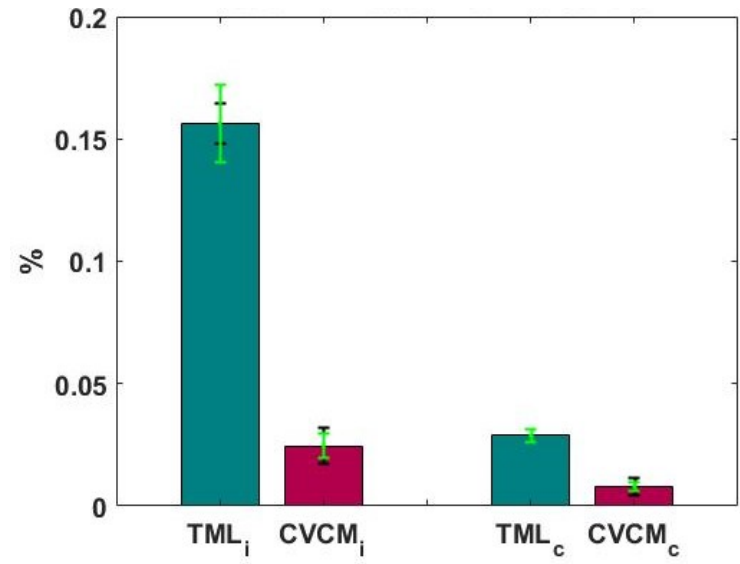

(c) RTV 566 Initial vs Control

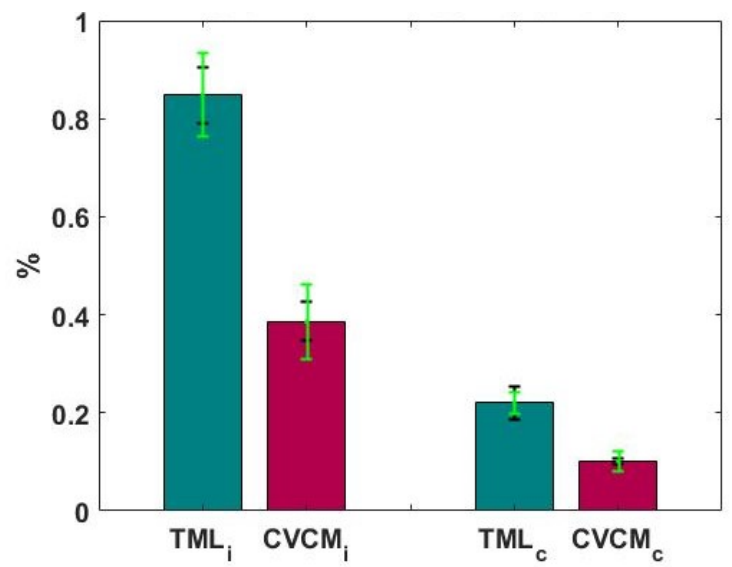

(b) Tflex Initial vs Control

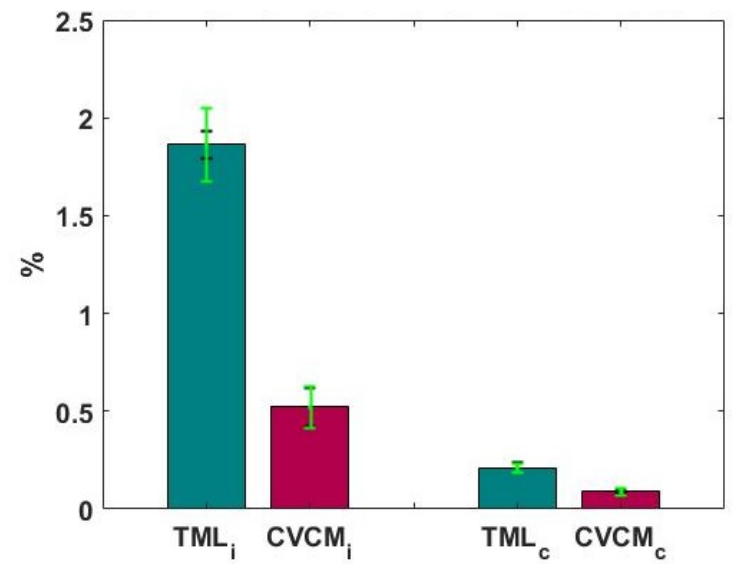

(d) NuSil R-2141 Initial vs Control

Figure 5.7: Silicone Materials TML and CVCM Outgassing Results for all Samples in the Initial Test Compared to Control Samples in the Final Test

calculated using eqs. (4.1) and (4.2). The average flux for all of the tests was $1.20 \pm$ $0.07 * 10^{16} \frac{\text { atoms }}{\mathrm{cm}^{2} * s}$ and the average fluence was $1.04 \pm 0.06 * 10^{21} \frac{\text { atoms }}{\mathrm{cm}^{2}}$ where the listed errors are plus or minus one standard deviation in the data.

Additionally, each of the tests was run thorough an independent samples t-test to determine if the results were statistically significant. Since there is such a small number of samples that can be tested in each run it was assumed that there would not be statistical significance with just a single test at the differences in TML and CVCM that were being observed. However, it was of interest to ultimately determine 
how many tests would be necessary to show statistical significance at a significance level of 5\%. For each of the tests, the individual TML values of the control group were compared to the TML values of the AO exposed group and the means of each group were compared using an independent samples t-test to obtain a single tailed p-value. This process was repeated for the CVCM values as well. It turned out that it was possible to have statistically significant differences between the means of the two groups after just one test which was surprising. The results are shown in tables 5.3 and 5.4.

Table 5.3: Independent Samples t-test Results for TML data

\begin{tabular}{|l|l|l|l|}
\hline Material & $\begin{array}{l}\text { Mean Control } \\
\text { TML }\end{array}$ & $\begin{array}{l}\text { Mean AO } \\
\text { TML }\end{array}$ & P-Value \\
\hline NuSil R-2141 Silicone & $0.218 \%$ & $0.152 \%$ & 0.000 \\
\hline Tflex Silicone & $0.254 \%$ & $0.066 \%$ & 0.045 \\
\hline RTV 566 Silicone & $0.046 \%$ & $0.035 \%$ & 0.000 \\
\hline CV-2566 Silicone & $0.099 \%$ & $0.094 \%$ & 0.155 \\
\hline M55 Graphite Fiber/ Composite & $0.044 \%$ & $0.058 \%$ & 0.043 \\
\hline EA 9360 Loctite Epoxy & $0.163 \%$ & $0.187 \%$ & 0.054 \\
\hline EN-11 Polyurethane & $0.133 \%$ & $0.092 \%$ & 0.015 \\
\hline
\end{tabular}

For each of these, there is strong evidence that the means are different if the $\mathrm{p}$ value is less than 0.05 , and some evidence that the means are different if the $\mathrm{p}$ value is less than 0.10. The results show that 3 of the four silicone materials (NuSil R-2141, Tflex, and RTV 566) showed a statistically significant smaller mean TML for the AO exposed samples compared to the mean TML for control samples. The p-value for the fourth silicone, CV-2566 was only slightly too large to have moderate evidence for the same conclusion. The other three materials all had strong or moderate evidence 
Table 5.4: Independent Samples t-test Results for CVCM data

\begin{tabular}{|l|l|l|l|}
\hline Material & $\begin{array}{l}\text { Mean Control } \\
\text { CVCM }\end{array}$ & $\begin{array}{l}\text { Mean AO } \\
\text { CVCM }\end{array}$ & P-Value \\
\hline NuSil R-2141 Silicone & $0.062 \%$ & $0.056 \%$ & 0.148 \\
\hline Tflex Silicone & $0.101 \%$ & $0.044 \%$ & 0.000 \\
\hline RTV 566 Silicone & $0.022 \%$ & $0.023 \%$ & 0.392 \\
\hline CV-2566 Silicone & $0.032 \%$ & $0.022 \%$ & 0.000 \\
\hline M55 Graphite Fiber/ Composite & $0.020 \%$ & $0.053 \%$ & 0.094 \\
\hline EA 9360 Loctite Epoxy & $0.011 \%$ & $0.011 \%$ & 0.478 \\
\hline EN-11 Polyurethane & $0.008 \%$ & $0.008 \%$ & 0.386 \\
\hline
\end{tabular}

showing a difference in mean TML. The CVCM differences were not significant quite as often, with only the Tflex and CV-2566 showing strong evidence of a difference between the AO exposed mean CVCM and the control mean. The M55 composite did have moderate evidence supporting a difference in mean CVCM values. Interestingly, there did not appear to be a correlation between the TML trends and CVCM trends as a low p-value for the TML differences did not correspond to a low p-value for the CVCM differences.

\subsection{FTIR Spectroscopy}

The Fourier Transform Infrared (FTIR) spectroscopy was performed on three of the four silicone materials in order to see if there was an observable difference in chemical structure due to AO exposure. The only silicone material that was not tested was the NuSil R-2141 for practical reasons. That silicone was an adhesive that was cured on aluminum foil boats. The boats could not easily be removed from the samples and 
it would have interfered with the test, so that material was left out. The following plots of percent transmittance as a function of wavenumber allow an insight into the chemical structure of the material. Every peak that sharply dips down on the plot is an indicator of a different type of bond that is either vibrating, stretching, deforming, or rocking and different peak shapes and sizes correspond to specific bonds between molecules and elements. As an example, the FTIR response of a generic polydimethylsiloxane (PDMS, a common silicone) is shown in fig. 5.8.

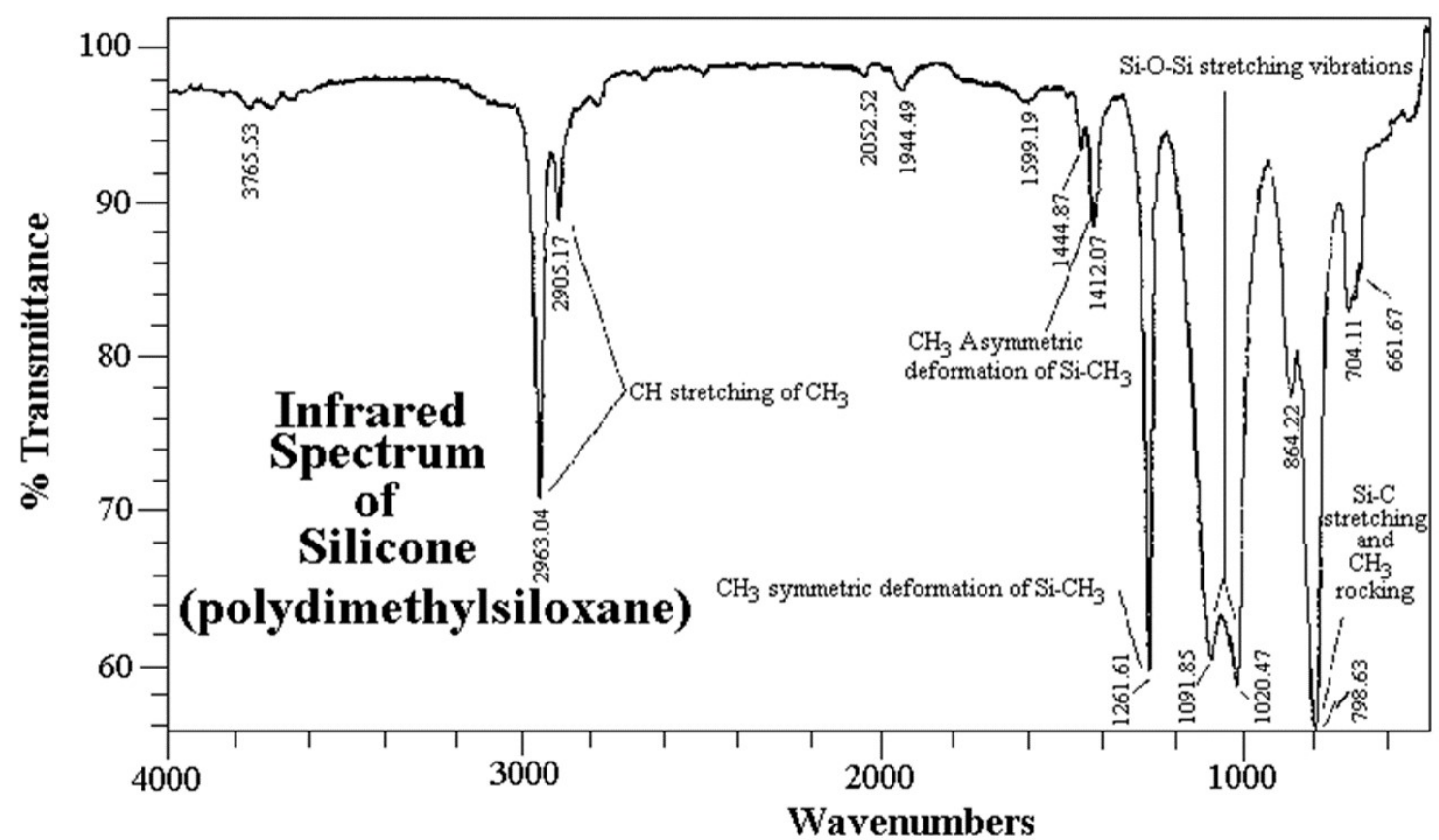

Figure 5.8: General Polydimethylsiloxane Silicone Transmittance Response to FTIR [28].

The figure even shows what each peak represents in the context of the silicon atoms, methyl groups, and carbon atoms. The motivation behind this testing was to see if there would be an explanation for the decrease in TML values for the silicone materials shown in the above results.

The FTIR results of the RTV 566 silicone are shown in fig. 5.9 with vertical lines at each of the peaks on fig. 5.8. It is clear that the RTV 566 is very similar to a 
generic PDMS as it exhibited very similar peaks at the same wavenumbers.

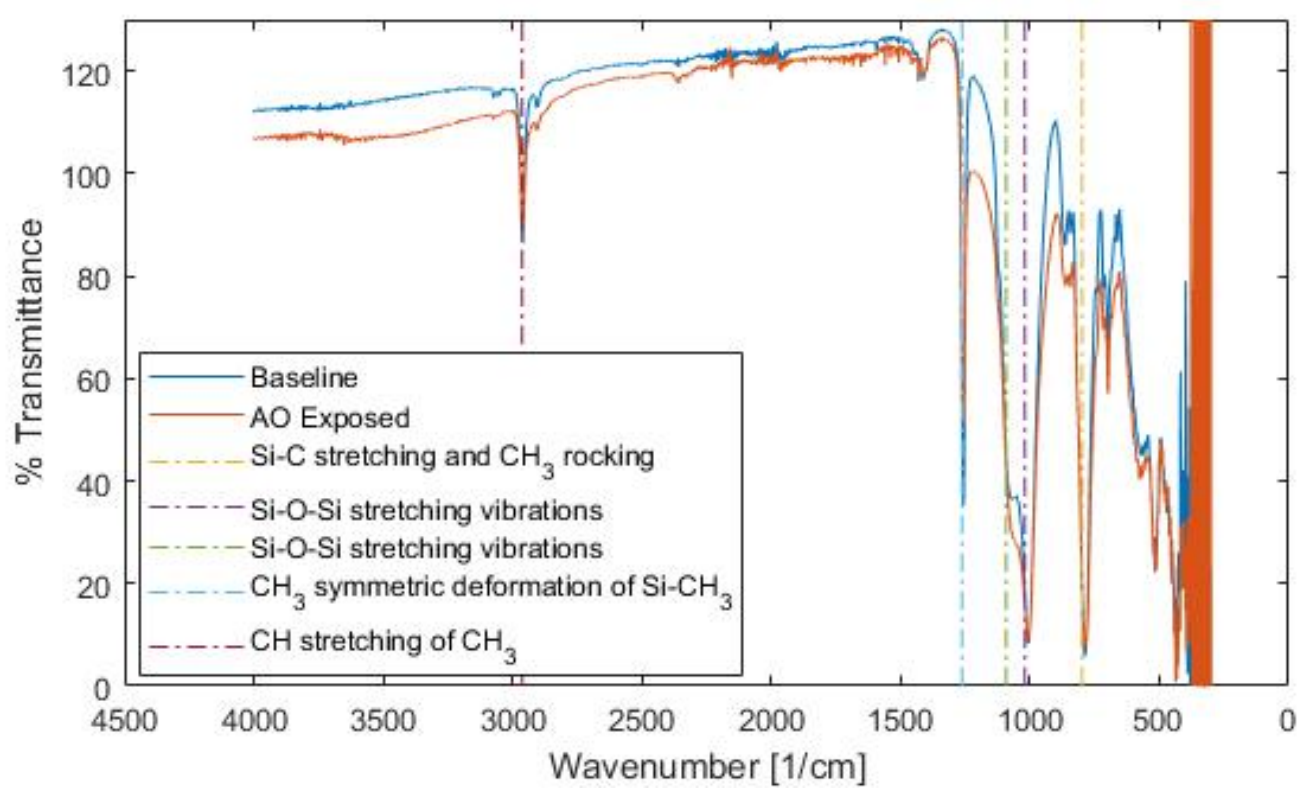

Figure 5.9: RTV 566 Silicone Transmittance Response to FTIR

Figure 5.10 shows the results of the CV 2566 silicone material with the same vertical lines as in fig. 5.9. Again there is a noticeable difference between the two peaks at the wavenumber of $1258 \mathrm{~cm}^{-1}$ and fig. 5.11 shows a closer look at that peak.

There are a few additional lines added to this plot that allow for some simple analysis to be performed. First it is important to note that the important thing to analyze on these plots are the depth and shape of the peaks as they compare to each other. Since the two plots do not return to $100 \%$ transmittance after the peak, there needs to be a way of measuring the depth of the peaks on each plot relative to the height of the nearby maximum transmittance points. Van der Meer establishes a robust method of performing this analysis by creating a smooth line continuum from the maximum transmittance points on either end of each peak [38]. The depth of these peaks are then measured relative to the continuum so that plots can be compared on an equal relative scale. Determining this continuum for the entire spectrum is a 


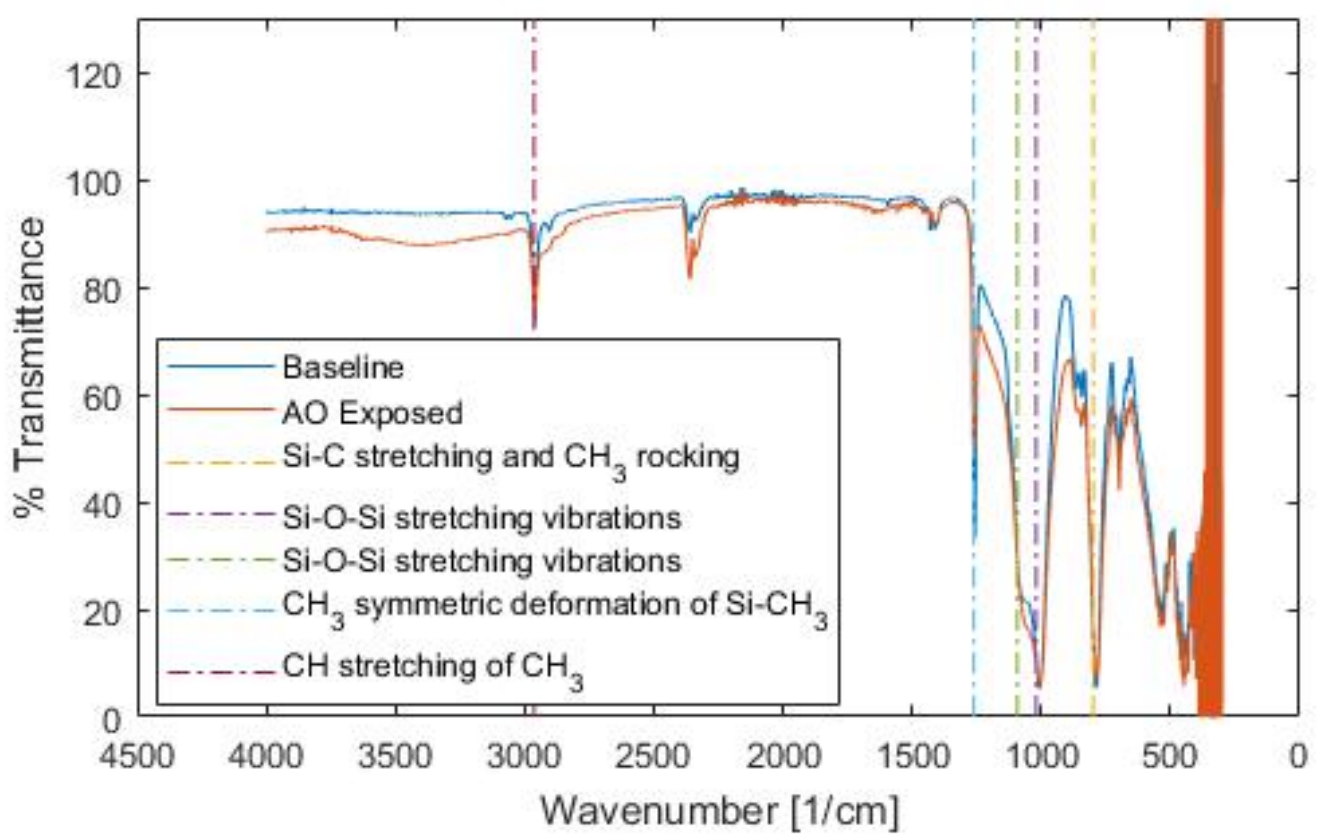

Figure 5.10: CV 2566 Silicone Transmittance Response to FTIR

complicated and difficult process done by advanced software programs that were not available for this thesis. Instead, a simplified continuum was generated, just for one peak of interest in each plot. This was done by fitting a third order polynomial just to the data near the peak of interest. It is difficult to see in the figure, but the main difference between the baseline and the $\mathrm{AO}$ exposed sample is at a wavenumber of $1258 \mathrm{~cm}^{-1}$. Figure 5.11 zooms in to that peak on the plot for a better view, and includes the approximate continuum curve for each case.

Creating a continuum for each plot allows for the comparison of different plots by calculating a new relative transmittance value using the equation,

$$
T_{\text {new }}=\frac{T_{\text {data }}}{T_{\text {cont }}} * 100 \%
$$

where $T_{\text {new }}$ is the new relative transmittance, $T_{\text {data }}$ is the transmittance values of the experimental data, and $T_{\text {cont }}$ is the transmittance values of the continuum. 


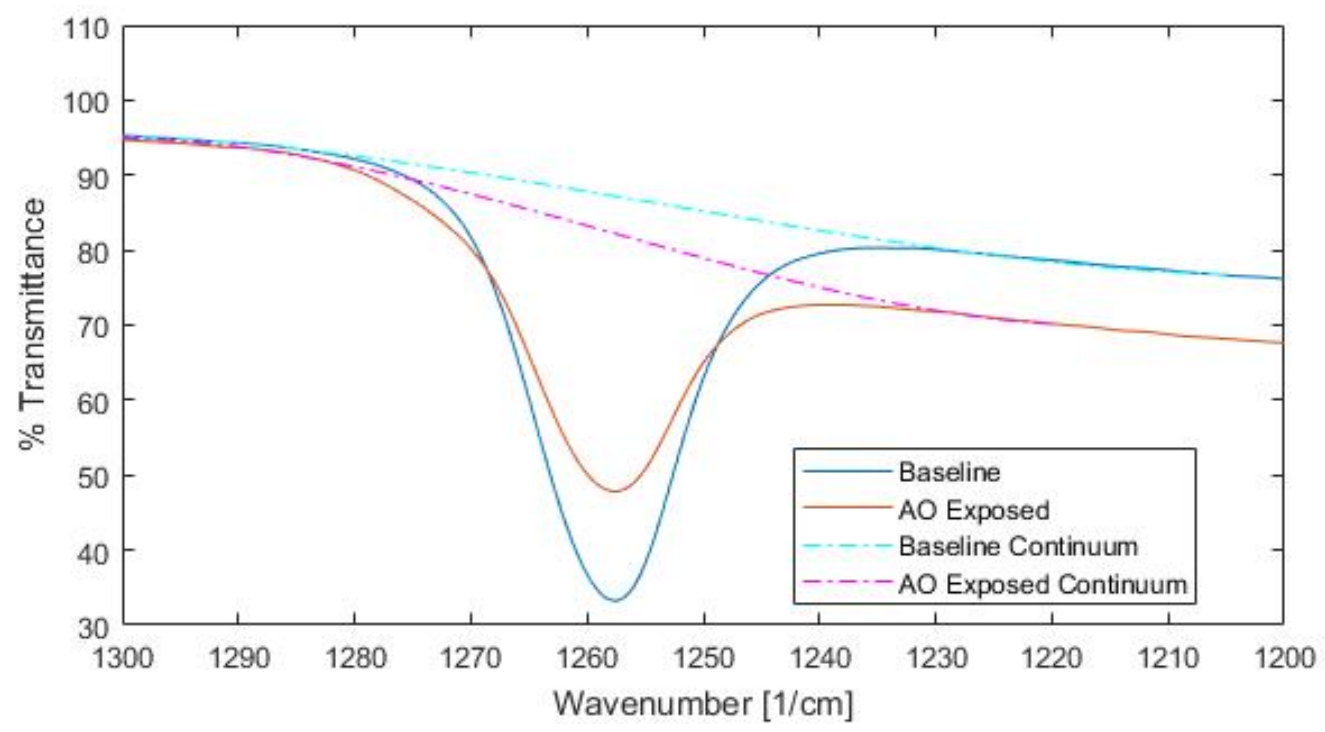

Figure 5.11: Zoom View of CV 2566 Silicone FTIR peak at a wavenumber of $1258 \mathrm{~cm}^{-1}$

Figure 5.12 shows the new adjusted transmittance curves along with the line showing how the depth of each peak is measured and compared on an equal scale.

From this figure, it is clear that the peak depth for the original sample of material is larger than the peak depth for the $\mathrm{AO}$ exposed sample. This change in peak depth at this location means there was less symmetric deformation of the bond between silicon and methyl groups according to fig. 5.8. The same method was used to analyze the results of the RTV 566 silicone as shown in figs. 5.13 and 5.14.

Lastly the third material tested for FTIR was the Tflex silicone thermal gap filler which surprisingly did not have a spectrum similar to the other two as shown in fig. 5.15. There was a noticeable disappearance of a peak at roughly $2800 \mathrm{~cm}^{-1}$, however, it is unclear what that peak is associated with. Thus the FTIR results for this material did not result in any notable conclusions. 


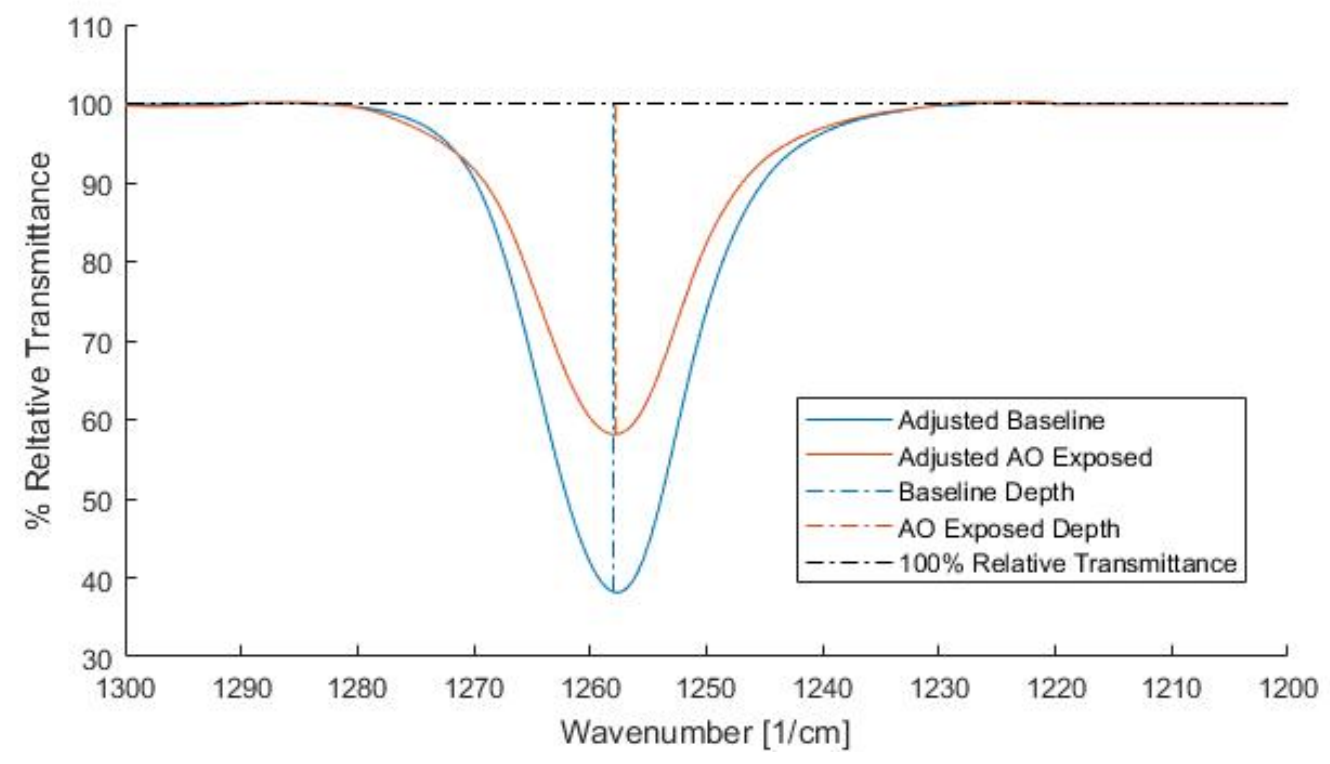

Figure 5.12: Zoom View of Adjusted CV 2566 Silicone FTIR peak at a wavenumber of $1258 \mathrm{~cm}^{-1}$

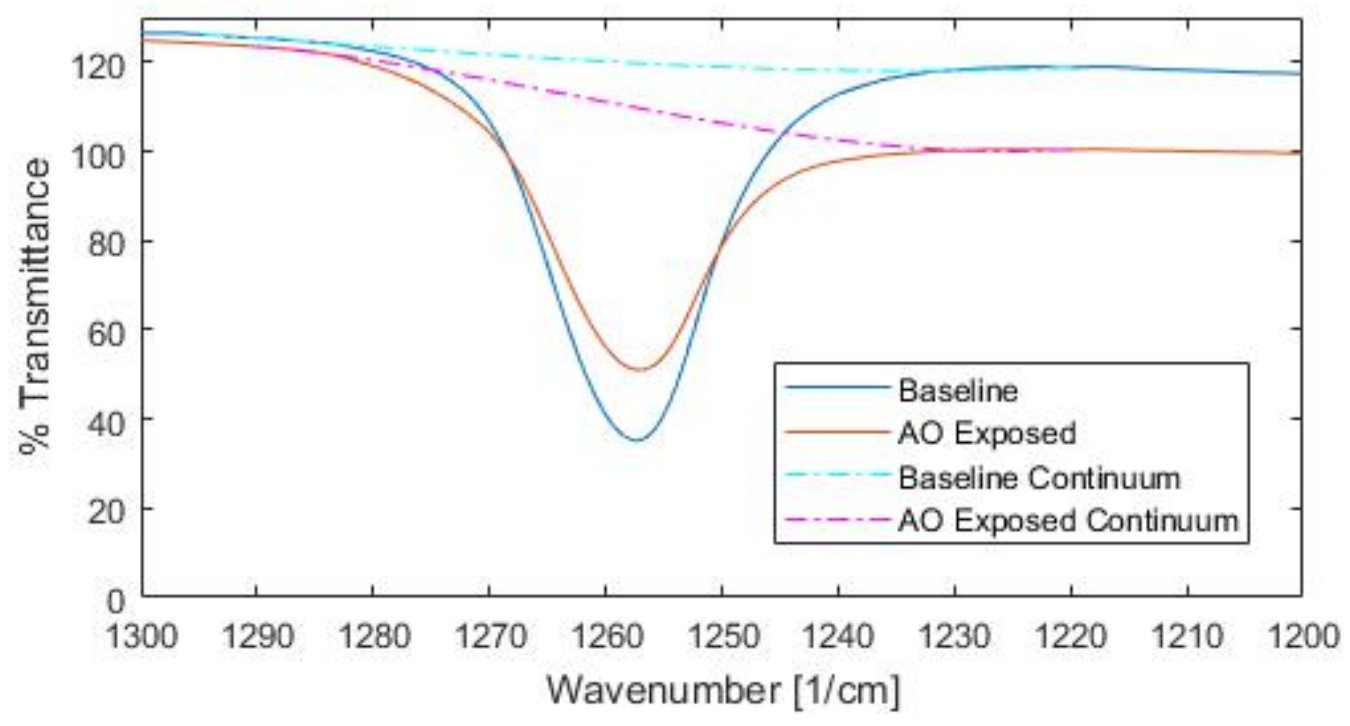

Figure 5.13: Zoom View of RTV 566 Silicone FTIR peak at a wavenumber of $1258 \mathrm{~cm}^{-1}$ 


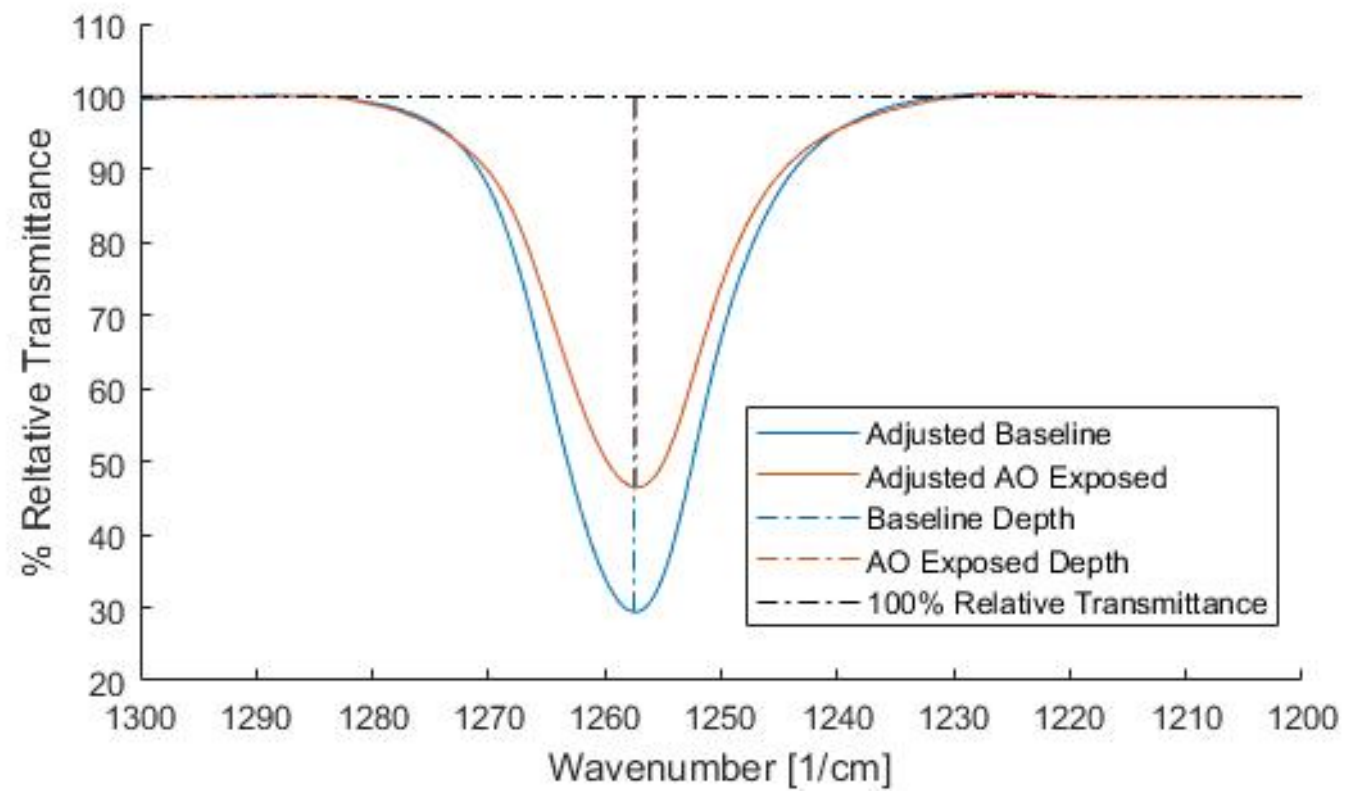

Figure 5.14: Zoom View of Adjusted RTV 566 Silicone FTIR peak at a wavenumber of $1258 \mathrm{~cm}^{-1}$

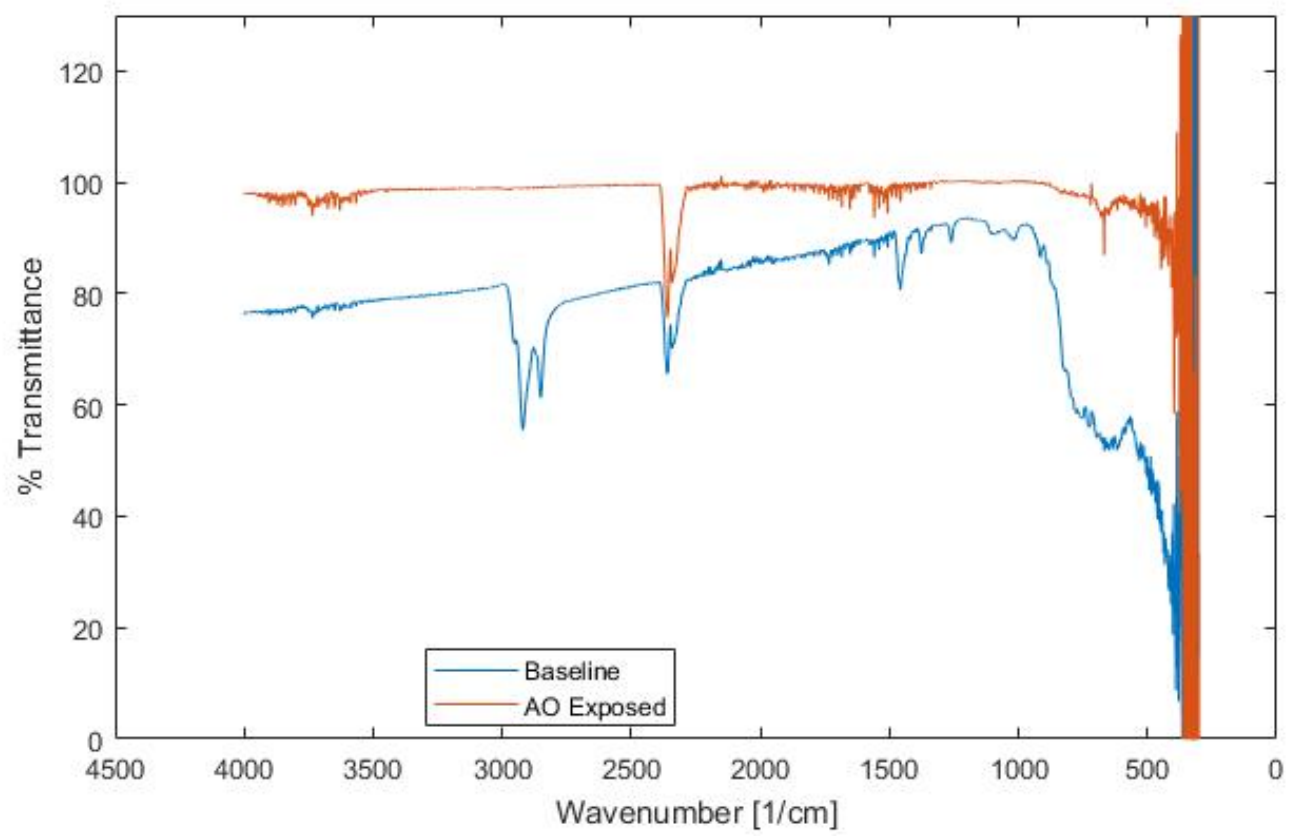

Figure 5.15: Tflex Silicone Transmittance Response to FTIR 


\section{Chapter 6}

\section{CONCLUSION}

The results from the tests with an increased sample number show that there is less variation in the data for tests run with more samples. The clear trend of smaller TML and CVCM values for $\mathrm{AO}$ exposed silicone samples is an interesting finding that supports other previous research related to silicones and AO exposure. In order to understand what is happening here, it is necessary to look at what a silicone is on a molecular level. The basic fundamental building block of any silicone is a siloxane chain which is a long chain of alternating silicon and oxygen atoms bonded together much like a carbon-based polymer chain. Silicon is tetravalent which means two oxygen bonds on a single silicon is not sufficient to fill its valence shell. All silicones have side chains, or additional groups that are bonded to each silicon atom in order to fill the valence shell. This is where manufacturers can get creative and by changing the side groups on the siloxane chain, the macroscopic properties of the resulting silicone can vary significantly. The most typical silicone is polydimethylsiloxane (PDMS) which is a standard siloxane chain with two methyl groups bonded to each silicon atom. A methyl group is $\mathrm{CH}_{3}$ and in the case of PDMS, the carbon is bonded directly to the silicon with the three hydrogen atoms bonded to the carbon atom.

When a PDMS silicone is exposed to AO, the methyl groups are oxidized and the surface layer of the silicone is gradually converted from a siloxane chain to $\mathrm{SiO}_{x}$, with $\mathrm{x}$ being close to 2 [6]. Other facilities that have tested silicones in ground based AO chambers and found silicones to crack and peel away on the exposed surfaces while forming $\mathrm{SiO}_{x}$ include another test by Bruce Banks in [7]. Perfect $\mathrm{SiO}_{2}$ is silica which is essentially glass (quartz to be precise), which is significantly more brittle than the flexible rubber of PDMS. The surface layer of the PDMS is not 
evenly converted to $\mathrm{SiO}_{2}$, however, and small irregularities in the material allow the $\mathrm{AO}$ to penetrate deeper and further, causing the surface layers to peel away from the underlying silicone. This results in more silicone being exposed to the $\mathrm{AO}$ and further conversion of the silicone essentially to silica. This process is referred to as the glassification of silicone. Another side group that is often used instead of methyl is phenyl. Phenyl is very similar to a benzene ring with the chemical formula $\mathrm{C}_{6} H_{5}$ and is much more stable than a methyl group. It is well known that this stability allows phenyl to be much more resistant to oxidation compared to methyl.

While the manufacturers of materials do not typically release the full chemical structure of their materials for proprietary reasons, the CV-2566 material datasheet does list the chemical structure as "dimethyl, diphenyl" [29]. This implies that the structure contains silicones bonded to either two methyl groups or two phenyl groups, but not necessarily with any particular concentration. There could be many more methyl groups than phenyl groups and there is no real way to know, but the larger concentration of phenyl groups, the more resistant to oxidation the material would be. This could explain why the CV-2566 material did not exhibit the same decrease in TML for the AO exposed group. Two of the other three silicone materials tested (the Tflex and the NuSil R-2141) did not list phenyl groups as part of their chemical structure on their respective datasheets. The NuSil R-2141 is a multipart silicone adhesive that lists many methyl, dimethyl, and even trimethyl variations of side chains, but no phenyl groups [30]. The Tflex, a thermal gap filler, is a reinforced silicone elastomer which is a silicone with a filler material to provide the reinforcement [20]. The third silicone material tested, RTV 566, actually does state on the datasheet that the chemical structure is "methyl, phenyl", which again, does not describe the ratio of phenyl to methyl groups [26]. The listing of the structure like this also suggests there could be some silicon atoms that are bonded to both a methyl and a phenyl group as opposed to two methyls or two phenyls which is interesting. 
The other results from the synergistic testing show that any effect of AO exposure on outgassing properties is material dependent. The EN-11 polyurethane showed a lower TML for the AO exposed samples. Some materials including the kapton samples experienced no significant effect while the M55 composite saw an increase in outgassing after $\mathrm{AO}$ exposure.

The comparison of initial TML and CVCM values, to later control sample values, shows that most outgassing takes place early on when a material is put into a vacuum. Due to the test procedure, the samples did not have much time between tests to regain some of that outgassed mass and water vapor. This lack of available mass to outgass is likely why the samples showed much smaller outgassing numbers during the second outgassing test. This puts the AO exposed vs control sample results into perspective, as the differences are not as large by comparison. Even though some of the changes in the outgassing values due to $\mathrm{AO}$ were noticeable and significant, they did not come anywhere close to the outgassing values seen during the first 24 hours under vacuum. This leads to the conclusion that while there may be some synergistic effects between $\mathrm{AO}$ and outgassing, those effects may not warrant any design revisions because the effects are so small and after a few weeks on orbit in a vacuum, the outgassing rate is so small that it is not a significant concern.

The results of the independent samples t-tests support the hypothesis that silicone materials have a lower TML value after AO exposure. This conclusion can be expanded on to say that $\mathrm{AO}$ exposure causes these silicone materials to outgas less because this was an experiment where the samples were randomly assigned to the control and $\mathrm{AO}$ exposure groups. The random assignment allows a cause and effect conclusion to be drawn. The same conclusion cannot necessarily be drawn about the CVCM values as only 2 of the 4 silicone materials had a significant difference between the $\mathrm{AO}$ exposed mean and control mean. In general, effects of $\mathrm{AO}$ on outgassing properties appear to be more material dependent. One should be more careful when 
generalizing these results to all silicone materials, however, as the materials tested were not chosen randomly from all spacecraft silicones. Instead they were the silicones most readily available for JPL to donate for testing.

The FTIR testing confirms the reasoning for the trends seen with the silicone materials showing lower TML after AO exposure. The decrease in the peak associated with symmetric deformation of the bond between silicon and methyl groups means that the AO exposed group had less methyl groups than the baseline sample. This is because the AO oxidized the methyl groups and formed the $\mathrm{SiO}_{x}$ layer on the surface (again, where $\mathrm{x}$ is near 2). If the $\mathrm{AO}$ had not altered the chemical structure on a molecular level, we would have expected to see little to no change in that critical peak depth, but the results clearly showed a substantial change. This result was verified for the CV-2566 and RTV 566 silicones. The FTIR did not show anything discernible about the presence or effect of phenyl groups on the two silicones as they both experienced a similar change due to AO. The Tflex could not be fully analyzed because it turned out it was not as similar to a generic PDMS silicone as previously thought and the critical peak depths were not associated with any of the known silicone peak bands. 


\section{Chapter 7}

\section{FUTURE WORK}

\subsection{ELI Chamber}

There are a number of improvements that can be made to the systems and procedures in this thesis in order to better the quality of future research. For ELI, one of the first things that should be looked at is the cryo pump. Ultimately the chamber needs to be able to reach a base pressure below $5 * 10^{-5}$ Torr which can be achieved a number of ways. The chamber leaks can be identified and reduced, the pump can be cleaned in an attempt to increase its pumping speed, the pump can be replaced altogether, or the chamber can be replaced altogether. There has already been some leak detection performed on the chamber and one significant leak source is the nitrogen gas feedthrough. The o-ring on the feedthrough was replaced but that did not reduce the leaks, and the feedthrough was even temporarily replaced with a feedthrough cover, but that did not seem to reduce the leaks either. It is possible that both of the only two feedthroughs that fit that port are inherently leaky and it would be worthwhile to buy a new replacement feedthrough and see if that remedies the problem. This was looked into briefly, but feedthroughs for this chamber can be difficult to find since they are not the standard CF or KF flanges. The pump could be replaced entirely by a new cryo pump or even a turbo pump but that is a very expensive option that should only be exercised once the others have been tried and proved ineffective. Cleaning the current cryo pump is likely to be the most effective method of lowering the chamber base pressure. Replacement parts can be relatively inexpensive for this procedure and they are available from Austin Cryogenics. Ultimately there should be a long term goal of replacing the ELI chamber with a newer chamber for outgassing 
that has more standardized feedthroughs that are easily replaceable, such as one with KF flanges.

The next major improvement that should be made on the system is the replacement of the thermocouples. One of the most important aspects of outgassing testing according to ASTM E595 is the ability to heat the samples to within a very specific temperature range. Without the ability to properly measure that temperature, there is no way to verify how close or how far away the system is from being able to meet the requirements of the standard. Currently the thermocouples have wires that are old with damaged sheaths and many feet of excess wire that make them difficult to troubleshoot. The thermocouples should be removed and replaced with either a new set of t-type thermocouples or surface mounted RTD's. A t-type thermocouple does not quite have enough precision to measure within \pm 1 degree at $125^{\circ} \mathrm{C}$, however it is just beyond that tolerance and it is what JPL and SSL both use in their systems. There are also a certain type of t-type thermocouples called special t-type that are more expensive and have more precision, so those are an option as well. Finally, surface mounted RTD's are a more expensive, more precise, and more permanent solution as they can be adhered to the sample bar thus saving some time in the transition phases of testing by eliminating the need to clamp the thermocouple ends between the cover plates and the sample bar.

Once a system is in place to more accurately measure the temperature on the sample bar, the next step would be to set up a system to automatically adjust the power supply to maintain the sample bar at $125 \pm 1^{\circ} \mathrm{C}$. This is standard for most systems in industry, as temperature conditions in the room of the chamber can affect the temperature of the sample bar. A previous student, Ryan Moskaluk, put together an Arduino with a temperature controller for this purpose. The controller adjusts the supply of current through the sample bar resistor based on the thermocouple feedback and it has an LCD screen that displays the temperature readings. The Arduino and 
controller are in the lab, and they just need to be assembled, tested, and tuned once correct thermocouple readings are no longer an issue.

Another improvement to the system that could be made is in the dessicator, where the samples are kept leading up to the test, and in transition periods between tests. Currently the dessicator is simply a tupperware box with a D'Addario Two Way Humidification System replacement pack, typically used in instrument cases, to maintain relative humidity at $50 \pm 5 \%$. There is no temperature control for the box even though that is one of the requirements outlined in ASTM E595. This improvement could potentially be very expensive. However, if the department ever decided it was necessary to give the labs temperature control, that would solve the problem. JPL does all of their testing in a room that is temperature controlled which allows them to forgo having a temperature controlled sample containment box.

Electrically, there are a few issues that need to be addressed, and improvements that could be made on the chamber. Ultimately, the goal with ELI would be to have the chamber run off of one 3-phase plug and one single-phase plug, with the power to each of the pumps and instruments being controlled from the instrument panel on the front of the chamber. At the time of this writing, Eli is operating on two 3-phase plugs and two single-phase plugs. The chamber hoist and the mechanical pump are wired to be powered by a single 3-phase plug, and the compressor runs on a second three phase plug. The hoist motor technically runs on 2-phase power and could, in theory, be switched to run off of single-phase power. In addition, a 3-phase plug could be added to the back of the chamber, like that on MAX, for the compressor to plug into and be powered by a single 3-phase power cord. Another electrical issue that came up during testing, and caused a test to be aborted, was a short in one of the cables coming out of the front of the control panel. These wires were hooked up to the electrical heater, and for whatever reason one of them shorted out one day causing the fuse to blow in the Variac power supply, thus bringing the 
sample bar back down to ambient temperature. It is not clear what caused this short, as the electrical wiring inside of ELI is very complicated and should only be altered by a trained professional with a deep understanding of the electrical inner workings of the chamber. Cody Thompson, the lab tech, took a look at the issue but was unable to come to any conclusions, so a second electrical feedthrough was installed as a temporary workaround so testing could continue.

\subsection{MAX Chamber}

The MAX chamber is a much more complete and better functioning system, and as a result, does not have as many changes or recommended renovations. One thing that would be a great re-addition to the chamber would be the deuterium VUV lamp. Max Glicklin used the lamp to see if there was a synergistic effect between AO and UV as the lamp is capable of emitting radiation between 115 and $200 \mathrm{~nm}$, one portion of the UV spectrum. It has been determined that the lamp simply needs a new bulb and Dr. Abercromby is working with a company to replace it.

Another significant addition that would solve the base plate temperature issue in MAX would be a water cooled base plate. Temperature can have a large effect on the outgassing and mass loss of a material in a vacuum, and the temperature on the base plate of MAX can get very hot. For this research, any time the base plate got over $60^{\circ} \mathrm{C}$ the test was aborted. This happened infrequently, but that is still a high temperature, and lower temperatures definitely still have an effect on the mass loss of the kapton witness sample, thus causing a slightly higher calculated effective flux and fluence. Swapping out the base plate for one with a welded pipe running along the bottom would be somewhat of a major overhaul of the MAX architecture, but it would be quite worth it to avoid having to abort the occasional test. A water cooling unit similar to the one on ELI could be purchased for a few hundred dollars and the 
feedthroughs could be put in place for it to pump water through a new base plate.

Recently there has been an issue with the mechanical pump running inconsistently during its first 20 or 30 minutes of operation. This causes an issue where the chamber pressure fluctuates within a roughly 30 mTorr range as it cycles up and down, making it impossible to set the pressure to $175 \pm 10 \mathrm{mTorr}$. This issue resolves itself once the pump has been allowed to run for about a half hour, but it should be looked into. There are also some interesting issues with the pressures in the roughing lines that may or may not be related. There are two convectron gauges on MAX, one at the chamber and another in the cryo roughing line. During normal operation, the cryo roughing line should be sealed off and it should not be pumping down, but nevertheless it does pump down, just at a much slower rate than the chamber. It reaches a pressure about 50-75 mTorr higher than whatever the chamber is at, yet when the chamber is vented, it does not get vented. It stays at a few hundred mTorr and it can stay like that for weeks. It seems that there is some sort of connection between the chamber roughing line and the cryo pump roughing line and it may be that the cryo roughing valve is not creating a proper seal so that should be investigated and fixed.

The last thing that needs to be fixed on MAX is the length of the threaded steel rods in the chamber that set the height of the electrode and DSS. The interior of the chamber was disassembled before this research and when it was put back together, the ground plate did not sit as low in the chamber as before. As a result, the electrode and DSS needed to be lifted higher, and there is now about and inch or so of both threaded steel rods that is exposed. These should be cut to the proper length so they do not reflect RF energy or become a potential load in the system. 


\subsection{Microbalance Scale}

As for the microbalance, the scale is a very new addition to the system and the scale drift issues should continue to be looked into and experimented with. A trip to SSL later on during the course of this research revealed that they use a Z-stat 6430 ionizer fan as the standard device to get rid of static buildup on their devices. This may be worth purchasing as they are inexpensive and its performance is likely superior to the anti-stat gun. Also, a set of standard weights should be purchased for the scale. JPL includes a weighing of their standard weights in their outgassing procedure described in section C.10. They record the mass of the standard weights before each test to verify the scale is working properly.

\subsection{Synergistic Testing}

There are a number of things that could be researched and tested further that would improve future synergistic testing involving outgassing, atomic oxygen, and the materials involved in this thesis. The first thing that should be tested is the AO exposure gradient in MAX. If the gradient could somehow be quantified and shown to be consis-

tent and predictable then that would eliminate the unknown of if samples experience the same AO exposure at different locations on the ground plate. One idea for such an experiment would be to cover the entire ground plate in kapton squares and then the mass loss of each square could be measured so the flux and fluence at each location could be determined. Testing could also be done with a set of strips of the kapton tape covering the entire ground plate to see qualitatively if the electrode really does produce an outline of higher AO exposure on the kapton. Once this is done, the next goal would be to use this information to come up with a consistent way to test long strips of kapton synergistically. 
Additional FTIR testing of silicones should be performed to see what, if any, silicones are actually chemically forming new bonds or if the siloxane chain is simply shortening to cause the brittleness. The FTIR testing could also be performed on other sample types to see if any of them are forming any new types of bonds or protective layers.

A sample box for use in MAX that can isolate for different specific effects like $\mathrm{UV}$, charged particles, and $\mathrm{AO}$ would be a great addition to the synergistic testing abilities of the space environments lab. Something like that used in reference [23] could be a good starting point for such an apparatus. This could allow for certain samples to be exposed to one or two environments at a time, while shielded from the others. This is essential in an AO plasma chamber like MAX that produces many secondary effects, along with the AO, that may be reacting in unknown ways with some materials.

As a recommendation, any future synergistic testing should be performed with a single material at a time, and the maximum number of sample slots in ELI be used at once. This will provide the best chance of the results having statistical significance and small error bars. Testing one material at a time will also eliminate the possibility of one material outgassing contaminants onto another during the $\mathrm{AO}$ exposure test (this would also be an issue in the outgassing test in ELI if the samples were not isolated in their respective sample chambers). 


\section{References}

[1] American Society for Testing and Materials, West Conshohocken, PA. ASTM E595-93: Standard Test Method for Total Mass Loss and Collected Volatile Condensable Materials from Outgassing in a Vacuum Environment, 1993.

[2] American Society for Testing and Materials, West Conshohocken, PA. ASTM E2089-00: Ground Laboratory Atomic Oxygen Interaction Evaluation of Materials for Space Applications, 2006.

[3] B. Banks, J. Backus, M. Manno, D. Waters, K. Cameron, and K. de Groh. Atomic oxygen erosion yield prediction for spacecraft polymers in low earth orbit. NASA.

[4] B. Banks, K. de Groh, and S. Miller. Low earth orbital atomic oxygen interactions with spacecraft materials. NASA.

[5] B. Banks, K. de Groh, and S. Miller. Low earth orbital atomic oxygen interactions with materials. NASA Glenn Research Center, 2004.

[6] B. Banks, K. deGroh, S. Rutledge, and C. Haytas. Consequences of atomic oxygen interaction with silicone and silicone contamination on surfaces in low earth orbit. International Society for Optical Engineering, 1999.

[7] B. Banks, S. Rutledge, E. Sechkar, T. Stueber, A. Snyder, K. de Groh, C. Haytas, and D. Brinker. Issues and effects of atomic oxygen interactions with silicone contamination on spacecraft in low earth orbit. Materials in a Space Environment, Protection of Materials and Structures from the LEO Space Environment, 2000. 
[8] P. Chen. Contamination effects due to space environmental interactions. 39th Aerospace Sciences Meeting \& Exhibit, 2001.

[9] Cytec. Conathane EN-11 Technical Data Sheet, March 2016.

[10] H. de Groh, B. Puleo, and B. Steinetz. Effects of atomic oxygen and grease on outgassing and adhesion of silicone elastomers for space applications. 10th International Space Conference on Protection of Materials and Structures From the Space Environment., 2012. NASA.

[11] J. Denver, B. Banks, K. de Groh, and S. Miller. Degradation of spacecraft materials. Handbook of Environmental Degradation of Materials, 717(763), 2012.

[12] M. Glicklin. Development of a ground based atomic oxygen and vacuum ultra violet radiation simulation apparatus. Master's thesis, California Polytechnic State University, 1 Grand Ave, San Luis Obispo, CA, 93401, June 2012. Master of Science in Aerospace Engineering.

[13] E. Grossman and I. Gouzman. Space environment effects on polymers in low earth orbit. Elsevier Science Ltd., 2003.

[14] E. Gurnee. The effects of atomic oxygen on the outgassing properties of spacecraft materials. Master's thesis, California Polytechnic State University, 1 Grand Ave, San Luis Obispo, CA, 93401, November 2014. Master of Science in Aerospace Engineering.

[15] T. Harding. private communication, October 2017.

[16] D. Hastings and H. Garret. Spacecraft-Environment Iteractions. New York, New York: Press Syndicate of the University of Cambridge, 1996. 
[17] R. Khasanshin, A. Timofeev, and M. Ivanov. Influence of space radiation on the outgassing rate of a patterned polymeric composite in vacuum. In Protection of Materials and Structures from Space Environment. American Institute of Physics, 2009.

[18] R. Khassanchine, A. Timofeev, A. Grigorevskiy, and A. Galygin. Influence of uv radiation on outgassing of polymeric composites. Journal of Spacecraft and Rockets, 43(2), March - April 2006.

[19] S. Koontz, K. Albyn, and L. Leger. Material selection for long life in low earth orbit - a critical evaluation of atomic oxygen testing with thermal atom systems. Journal of the IES, 1990.

[20] Laird Technologies. Tflex Technical Data Sheet, June 2011.

[21] Loctite. EA 9360 Technical Data Sheet, September 2013.

[22] D. Martin and C. Maag. The influence of commonly used materials and compounds on spacecraft contamination. Acta Astronautica, 30, 1993.

[23] S. Miller, B. Banks, and D. Waters. Investigation into the differences in atomic oxygen erosion yields of materials in ground based facilities compared to those in leo. ISMSE and ICPMSE, 2006.

[24] S. Miller, B. Banks, and D. Waters. Investigation into the differences between atomic oxygen erosion yields of materials in ground-based facilities and leo. High Performance Polymers, 2008.

[25] T. Minton. Protocol for Atomic Oxygen Testing of Materials in Ground-Based Facilities. JPL, Pasadena, CA, September 1995.

[26] Momentive. RTV 566 Technical Data Sheet, May 2012. 
[27] NASA, Academy of Program/Project \& Engineering Leadership (APPEL). Orbital Debis Management $\mathcal{E}$ Risk Mitigation.

[28] Spectral Database for Organic Compounds (SDBS). http://sdbs.db.aist.go.jp. National Institute of Advanced Industrial Science \& Technology. Accessed: 2017-11-02.

[29] NuSil. CV-2566 Technical Data Sheet, May 2014.

[30] NuSil. R-2141 Two-Part Adhesive Technical Data Sheet, November 2015.

[31] N. Penney, J. Wasowski, and C. Daniels. Temperature and atomic oxygen effects on helium leak rates of a candidate main interface seal. AIAA/ASME/SAE/ASEE Joint Propulsion Conference E Exhibit, 2010.

[32] V. Pisacane. The Space Environment and its Effects on Space Systems. Reston VA: American Institute of Aeronautics and Astronautics, 2008.

[33] S. Rutledge and B. Banks. Atomic oxygen treatment technique for removal of smoke damage from paintings. Materials Research Society, 1996. NASA Lewis Research Center.

[34] S. Rutledge, B. Banks, and M. Kitral. Comparison of space and ground based facility environmental effects for fep teflon. In 4th International Space Conference on Protection of Materials and Structures from the LEO Space Environment, Toronto, Canada, April 1998. NASA TM-1998-207918/REV1.

[35] K.-B. Shin, C.-G. Kim, C.-S. Hong, and H.-H. Lee. Prediction of failure thermal cycles in graphite/epoxy composite materials under simulated low earth orbit environments. Elsevier Science Ltd., September 1999.

[36] D. Thunnissen. private communication, August 2017. 
[37] A. Tribble. The Space Environment: Implications for Spacecraft Design. Princeton University Press, Princeton, New Jersey, 2003.

[38] F. van der Meer. Analysis of spectral absorption features in hyperspectral imagery. International Journal of Applied Earth Observation and Geoinformation, 5, 2003. 


\title{
APPENDICES
}

\author{
Appendix A
}

\section{LESSONS LEARNED}

Throughout this research, there were a number of things discovered and learned about the details and logistics of performing testing in the Space Environments Lab, and it would be a loss if those lessons were not passed on. This section outlines those lessons so that future work with this equipment or in this field can be more easily accomplished.

In the beginning of testing for this thesis (winter quarter of 2017) the cryo pump that was on ELI was capable of pumping the chamber down just below the required pressure of $5 * 10^{-5}$ Torr. However, early on in spring quarter of 2017 , that cryo pump broke during the middle of a test. It is still unknown what caused it to break and it needs to be sent in for repairs. In any case, a cryo pump was taken off of one of the other chambers in the lab (Big Green), as that chamber did not need to reach very low pressures for the work that was being performed in it at the time. When the cryo pump switch was made, it was discovered that both pumps are extremely dirty. Once the working cryo pump was installed back onto ELI, it was quickly clear that it could not quite pump down to the same vacuum level as before. It would reach a base pressure of between $1.0 * 10^{-4}$ and $4.0 * 10^{-4}$ Torr. These cryo pumps are very old and as particulates condense and accumulate in the pump, their pumping speed is significantly reduced. In a system with leaks, that will cause your base pressure to be higher. After speaking with Dan Goebel, a vacuum expert at NASA JPL, it was determined that it is common for cryo pumps that are old or poorly maintained to accumulate debris and particulate matter, and thus have a significantly reduced 
pumping speed. They generally will benefit from a thorough cleaning. The CTI Cryogenics manual outlines some of the procedures for cleaning a pump like this as it is possible to clean it yourself via a number of different tactics instead of sending it in to an expensive professional repair shop. The least intrusive way involves performing a "bake-out" of the cryo pump, whereby the chamber is evacuated with a mechanical pump, and the cryo pump is heated using a coil heater or strip heaters in some way to heat the outside of the pump. A more thorough cleaning job can be performed by removing the cryo pump entirely and disassembling the collector tree plates and cleaning them with IPA or ethanol. The CTI Cryogenics manual describes these processes in more detail. If, in the future, the pumps are not achieving a sufficient base pressure, the manual should be consulted for the proper cleaning procedure. The appropriate indium gaskets and other parts will need to be purchased to replace the existing ones that will likely tear during the disassembly of the pump. As a note, the cryo pumps should never be removed by one individual alone as they are quite heavy and they need to be kept upright with a protective cover on the opening to prevent any debris from falling in.

Once it was clear that the newly installed cryo pump on ELI could not achieve the same base pressure as the previous pump it became necessary to check the system for leaks in an attempt to find an fix some of them so a lower base pressure could be achieved. For this process, the Biomedical Engineering department lent out their helium leak detector. Specifically it is a Model 979 Series Helium Mass Spectrometer Leak Detector by Varian Vacuum Technologies. The leak detector is essentially a mechanical pump that evacuates the chamber and pulls the air through an instrument that can detect trace amounts of helium. Once the pump has pulled a high enough vacuum on the chamber of interest, the operator can spray small amounts of helium onto feedthroughs, seals, or other potential leak points on the exterior of the chamber. If there is a leak at that point, the small helium particles will be sucked into 
the chamber and then pumped through the leak detector, providing feedback to the operator. A full set of procedures for the leak detector can be found in section C.11. Using the apparatus on ELI revealed that ELI has many potential leak points. ELI is also a very difficult chamber to leak detect, as the feedthroughs are all located on the bottom plate of the chamber which is parallel to the ground. Since helium rises, it is very difficult to pinpoint a potential leak because any helium sprayed there will rise and spread out along the entire bottom surface of the chamber, thus potentially entering the chamber at any of the feedthroughs. A recommendation for anyone using the helium leak detector on ELI in the future would be to create a temporary makeshift seal around the nozzle so that individual feedthroughs can be isolated during testing. Ultimately, the leak detector did prove to be useful in finding a few leaks including one in the nitrogen gas inlet feedthrough. Unfortunately, the chamber is so old that it does not use standard KF or CF vacuum flanges. Instead, it has some through-holes in the chamber base plate and it has other feedthroughs that are adhered on with some sort of red adhesive. Luckily, the nitrogen gas feedthrough is of the former category, and the o-ring was replaced and a small amount of vacuum grease added to the seal to reduce the leak.

There are a number of intricacies that were discovered with the microbalance scale during the course of this research. The Mettler Toledo balance is a very precise instrument that is easily perturbed by small disturbance forces that other scales are not affected by. This was realized early on when the measurement would drift, sometimes slowly, sometimes rapidly, in one direction for minutes on end when weighing certain samples. This was initially very frustrating as it prevented all of the samples from being weighed within the first hour of removing them from the chamber. A Mettler Toledo representative suggested that scale drift can be caused by a number of factors including temperature gradients between the weighing pan and the sample, exposure of the scale to direct sunlight, or a buildup of static electricity. After ensuring the 
scale was indoors in a room with no windows and the samples were at the same temperature as the weighing pan, it was determined that a significant source of the drift must be due to static electricity. This made sense because the drift is non-existent with small metal samples but very apparent with dielectric materials like kapton. To solve this problem, the Milty Zerostat 3 deionizing gun was purchased to be used with the scale in the lab. Any time the samples are being weighed that have the tendency to accumulate static it is a good idea to use the antistat gun. Many different ways of using the gun were tested and researched, as there is no official instruction manual that comes with the device. After some experimentation, the most effective way to use the device was determined and is laid out in section C.13. Samples that generally benefit from this treatment are the long strips of kapton and kapton coated materials. In order to weigh these samples they had to be rolled up and held together with a small copper wire loop once the scale had been tared to account for the weight of the copper wire loop. This act of rolling the samples while wearing gloves tends to cause a buildup of static on the samples, and it is difficult to avoid. These samples may need multiple repetitions of the deionization process before the scale drift slows down.

MAX is a delicately balanced system, and when it loses its matching point, it can be difficult to deal with. This takes practice to deal with in order to bring the reflected power back to zero. This paragraph details some observations that may assist future operators with getting the reflected power to settle to zero. As a baseline, when the AO is first turned on, the tune almost always settles on 33 or $34 \%$, while the load usually settles between 54 and $69 \%$. If the matching network is trying to force the capacitors to values that are far outside this range or the system is unable to find a stable point and continues to oscillate then turn the system off, re-check the setup and ensure all of the proper steps have been followed. Often times the system will find a matching point, but with 1 watt of reflected power. This can almost always be 
solved by manually adjusting the load until there is zero reflected power. This usually holds true whenever, during operation, there is 1 watt of reflected power. The phase and magnitude can sometimes be indicators of which way the tune and load need to be adjusted. If the phase is greater than 45 or $50 \mathrm{mV}$ then that can be an indication that the tune needs to be manually increased one notch. If phase is below -45 or $-50 \mathrm{mV}$ then tune may need to be decreased one notch. The same holds true for the relationship between magnitude and load. If the phase and magnitude are adjusted too far in one direction then the system may force itself to stabilize with 1 watt of reflected power. If that is the case, simply manually adjust tune or load so that there is zero reflected power and leave tune or load (whichever you adjusted) in manual mode while you manually adjust phase or magnitude back to $0 \pm 25 \mathrm{mV}$.

After a couple hundred hours of testing on MAX, an issue arose where, during testing and without warning, the matching network would suddenly lose the stable point for the adjustable capacitors. The matching network would begin to cycle through many different values of tune and load. Meanwhile, the forwarded power would drop in order to prevent significant reflected power which resulted in an alarming flickering of the light coming from the chamber due to the decrease in forwarding power not being able to sustain a plasma. This issue did not automatically solve itself, and even manual adjustment of the matching network couldn't allow it to find a stable point, so the system had to be shut off and restarted. It was determined that this issue was caused by tarnished ground straps. As discussed earlier, RF energy travels according to the skin effect, therefore any surface discontinuities on the grounding straps in the chamber will reduce their conductivity. This tarnish builds up over time after many hours of operation and it is imperative that it be cleaned off regularly in order to maintain a properly functioning system. The ground straps can be easily cleaned by removing them from the chamber and rubbing them with a fine grit sandpaper $(220$ or 500 grit) and vinegar, or by scrubbing them with a Scotch-Brite pad until the 
surface is clean and shiny.

There were additional issues when testing the long kapton strips that were problematic for each of the chambers and the microbalance scale. The only way a sample of that type can meet the mass requirement of greater than $200 \mathrm{mg}$ is if it is cut in a long strip so that it can be rolled up or if it is cut up into many small squares and stacked on top of each other to fit in the sample chambers in ELI. There is no significant reason to choose one method over the other, however it is easier to keep track of a single piece of material when moving things around and transitioning chambers, as opposed to many small squares of material. In addition, the small squares are tedious for testing in MAX as they will all need to be laid out so they can all be exposed to AO. The long strips are also difficult to deal with in MAX, however, as they do not lay flat, so they must be taped down with aluminum tape. This does cover some of the sample exposure area, but it is necessary and should not affect the results much so long as each of the strips has roughly the same amount of area covered by the tape. Once during testing, one of the strips popped out from beneath one end of the tape holding it down, and its naturally bowed shape caused it to stick up and get very close to the electrode. This in turn caused the system to lose the matching point for the AO plasma, and the test had to be aborted so the sample could be re-taped. When working with these materials it is more important to have them securely taped down than to have the minimum amount of area covered by the tape.

One of the biggest lessons learned in this work is that there are acceptable changes and modifications that can be made to the testing procedure of ASTM E595. These are done out of practicality for the system in mind, and while they do contribute to the error between different testing facilities, they allow for many facilities to collect data that otherwise wouldn't. The ELI chamber is no exception, as there are many modifications to the ASTM standard for this system. This is acceptable so long as the modifications are properly documented for other researchers to make note of 
when looking at the data from our facility. Many of the compromises and changes were already discussed in section 3.1, but there are a few more that have not been mentioned. The ASTM standard calls for a molecular sieve (5A or equivalent) in order to remove moisture from the nitrogen that the ELI chamber is vented with at the end of each test. A conversation with Nataly Chen at JPL revealed that this is not necessary, as the purified nitrogen usually has very little moisture in it. In fact, JPL used to vent their chamber with atmospheric air which they deemed acceptable until somewhat recently, when they switched to back-filling with nitrogen. SSL also does not use a molecular sieve to filter their nitrogen, therefore it is a reasonable procedure modification. ASTM E595 also calls for the chamber to be pressurized at the end of the test to 860-970 Torr which is 100-200 Torr above atmospheric pressure. The ELI chamber is not designed to be pressurized as the lid can be forced upward allowing the pressure to equalize. Instead, ELI should be pressurized to about 200 mTorr with nitrogen, and then once the sample bar has cooled, the chamber can be fully vented back to atmospheric pressure using the chamber vent valve which will prevent over pressurization. This modification is one that JPL uses as well, as their chamber, shown in fig. A.1, is not designed to be pressurized. 


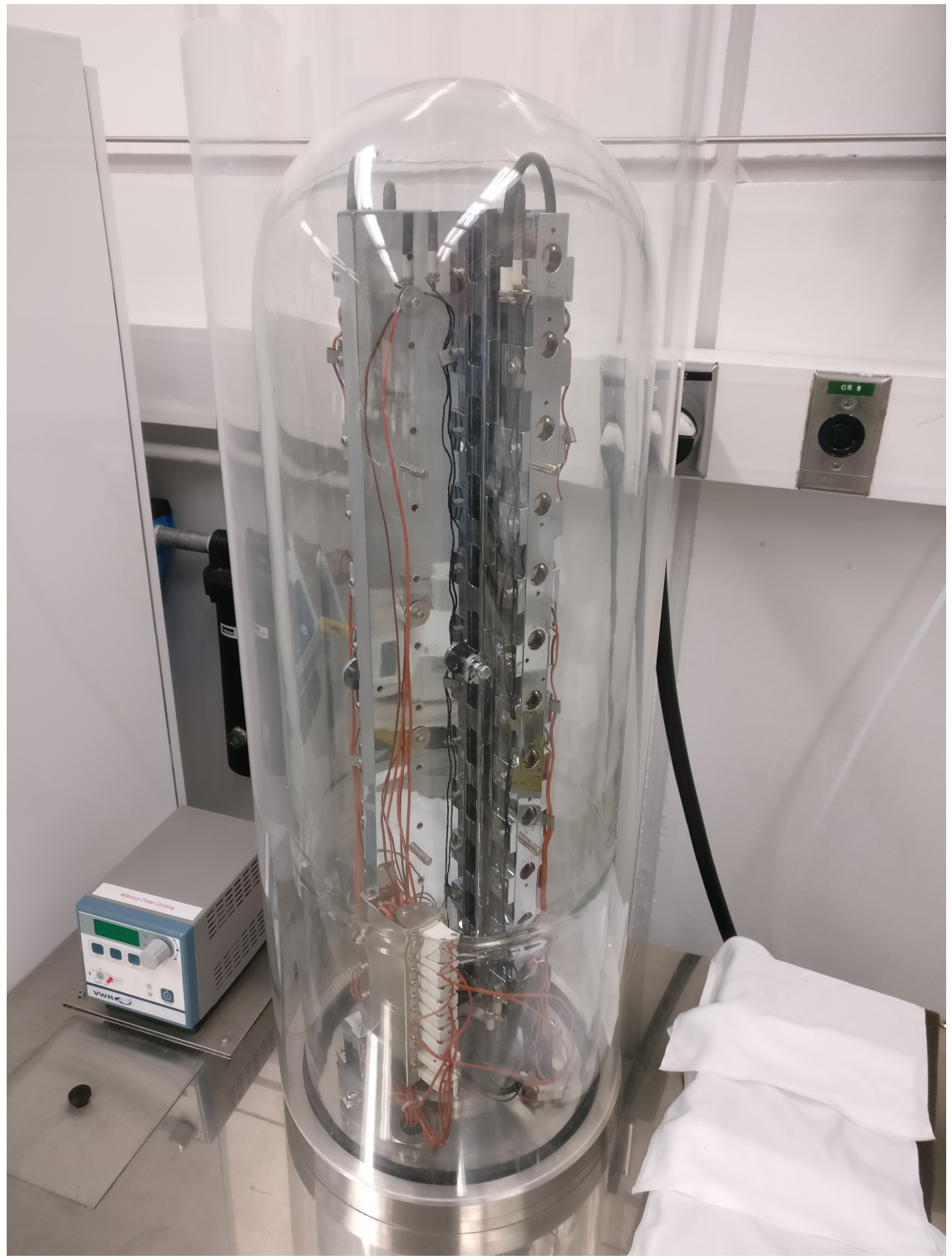

Figure A.1: NASA JPL outgassing chamber for ASTM E595 Testing. 
Appendix B

ADDITIONAL SAMPLE PICTURES

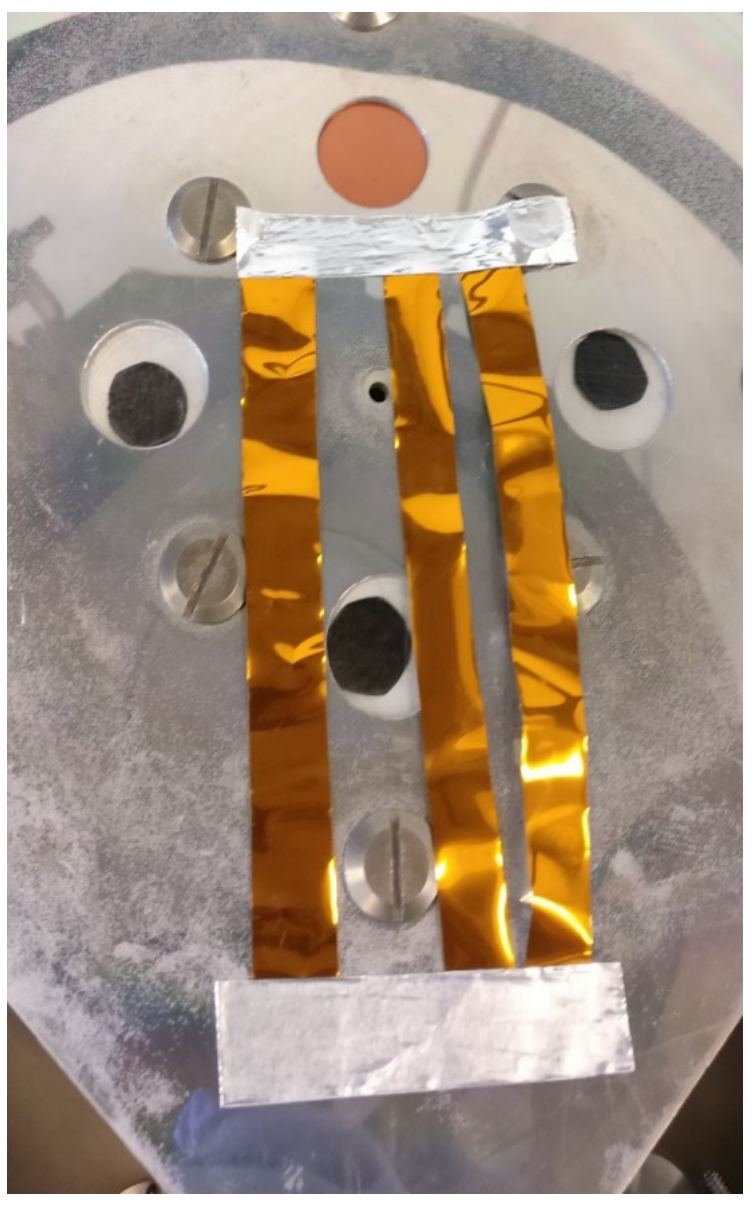

(a) Graphite composite disks and 2 mil Kapton strips before $\mathrm{AO}$ exposure

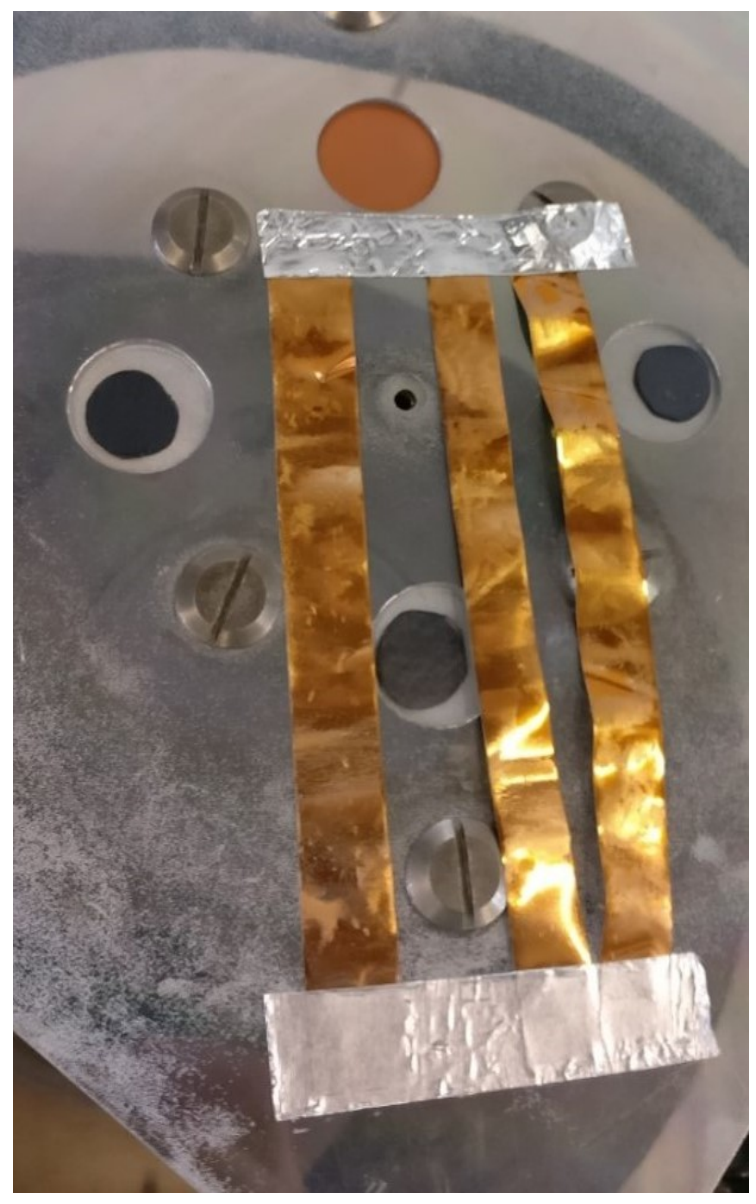

(b) Graphite composite disks and 2 mil Kapton strips after $\mathrm{AO}$ exposure

Figure B.1: Graphite composite disks and 2 mil Kapton strips before and after AO exposure with a Kapton $\mathrm{HN}$ witness sample in the top center 


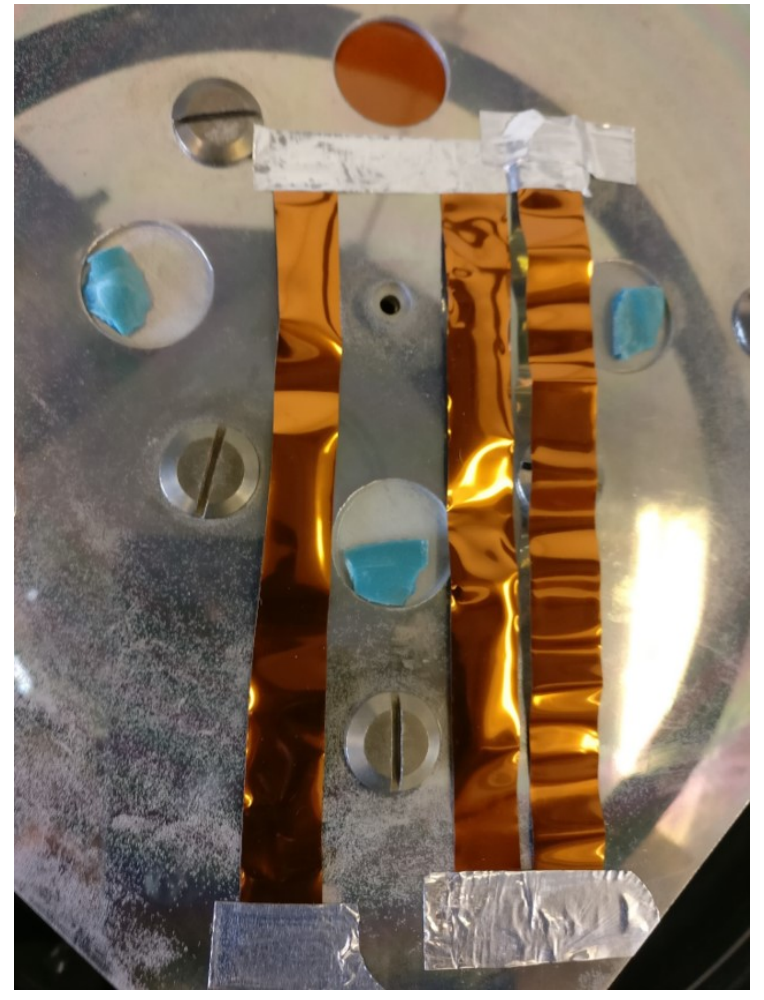

(a) 3 mil Kapton and EA 9360 before AO exposure

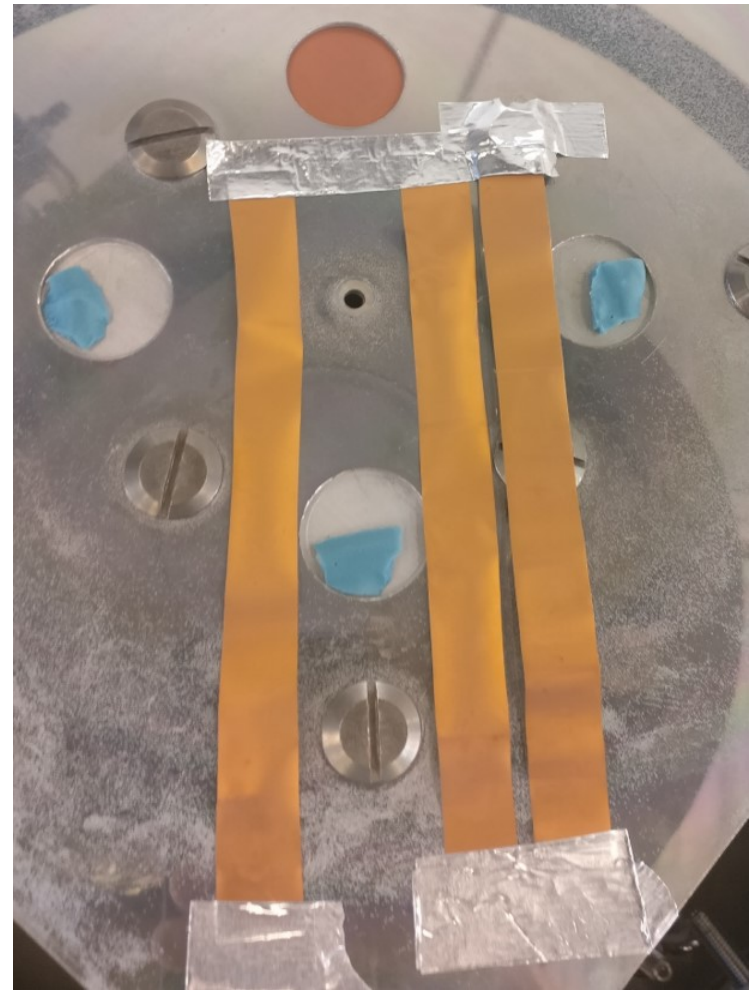

(b) 3 mil Kapton and EA 9360 after AO exposure

Figure B.2: 3 mil Kapton strips and EA 9360 Loctite adhesive (blue pieces) before and after AO exposure with a Kapton HN witness sample in the top center 


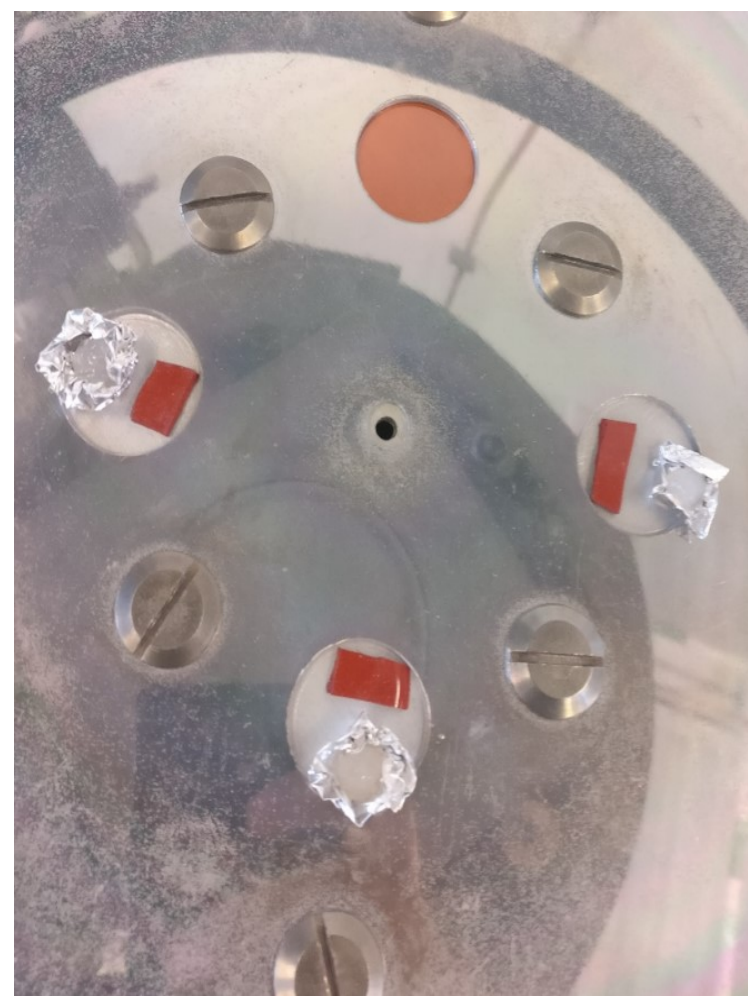

(a) NuSil R-2141 and CV-2566 before AO exposure

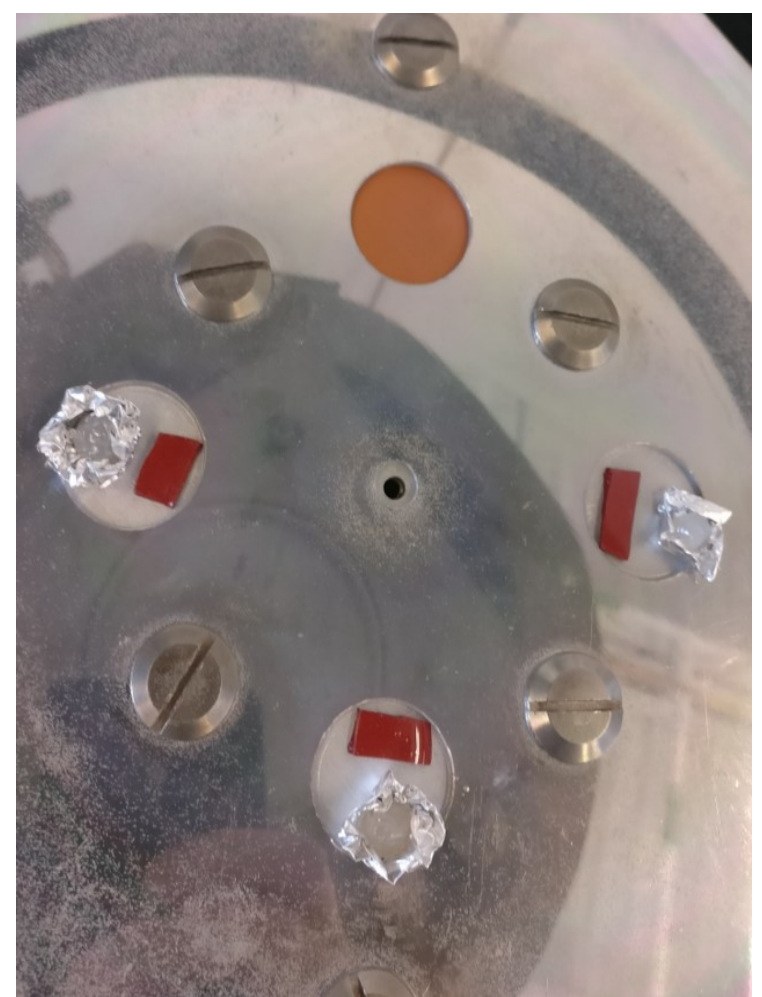

(b) NuSil R-2141 and CV-2566 after AO exposure

Figure B.3: NuSil R-2141 two-part silicone adhesive (white samples on aluminum boats) and CV-2566 silicone rubber (red rectangles) before and after $\mathrm{AO}$ exposure with a Kapton $\mathrm{HN}$ witness sample in the top center

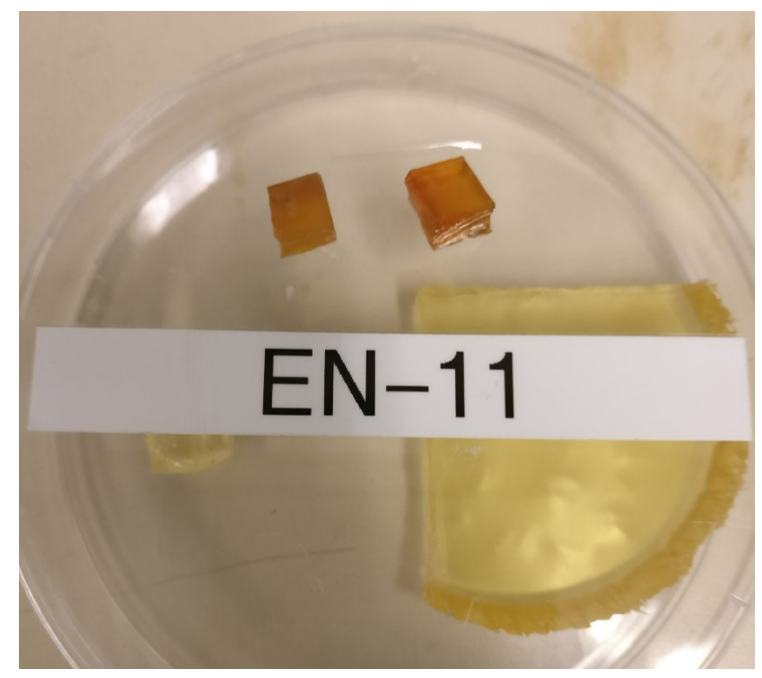

Figure B.4: EN-11 Polyurethane samples untested (yellow transparent large piece), outgassed (top right transparent orange cube), and outgassed \& AO exposed (top left translucent orange cube) 


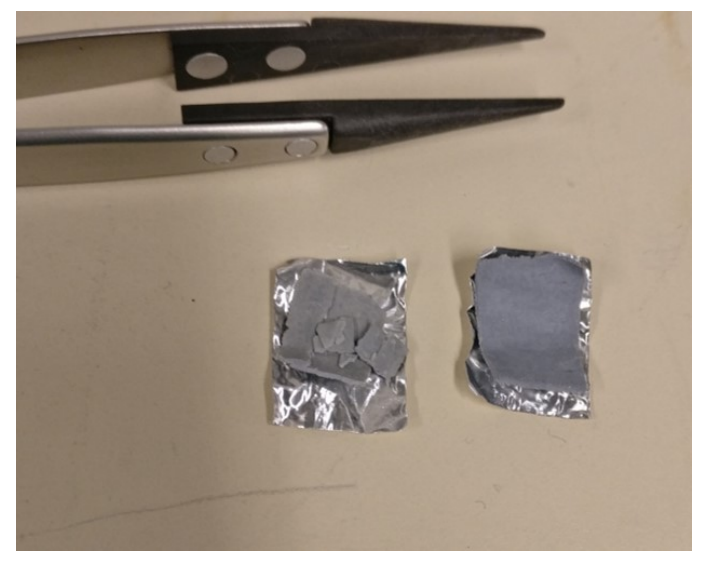

Figure B.5: Tflex samples AO exposed and outgassed (left), and outgassed (right)

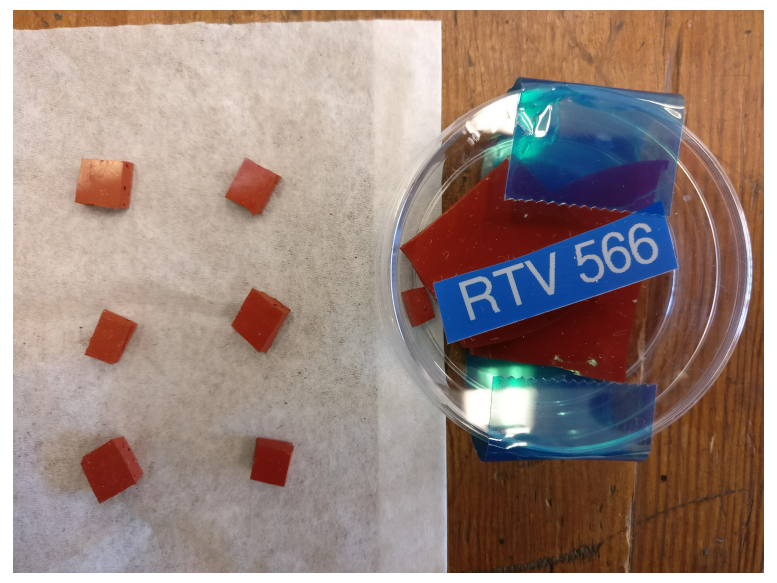

Figure B.6: RTV 566 silicone rubber samples untested (right), outgassed and AO exposed (center column), and outgassed (left column)

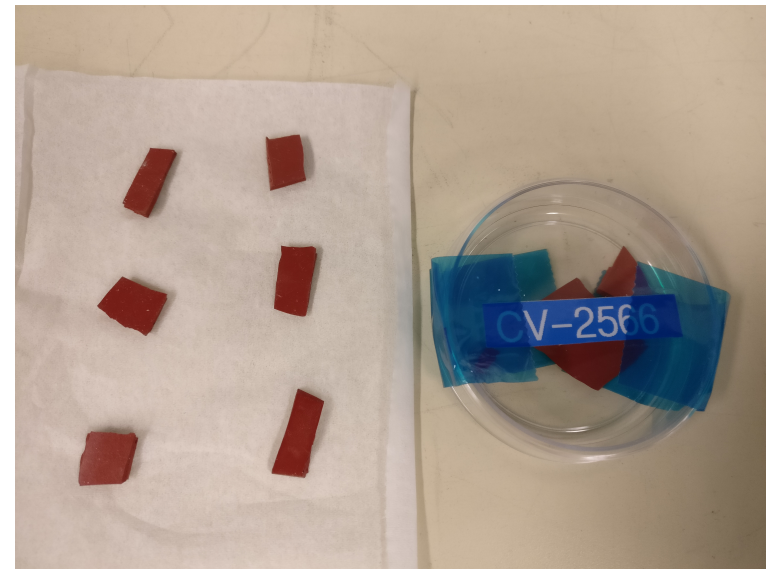

Figure B.7: CV-2566 silicone rubber samples untested (right), outgassed and AO exposed (center column), and outgassed (left column) 


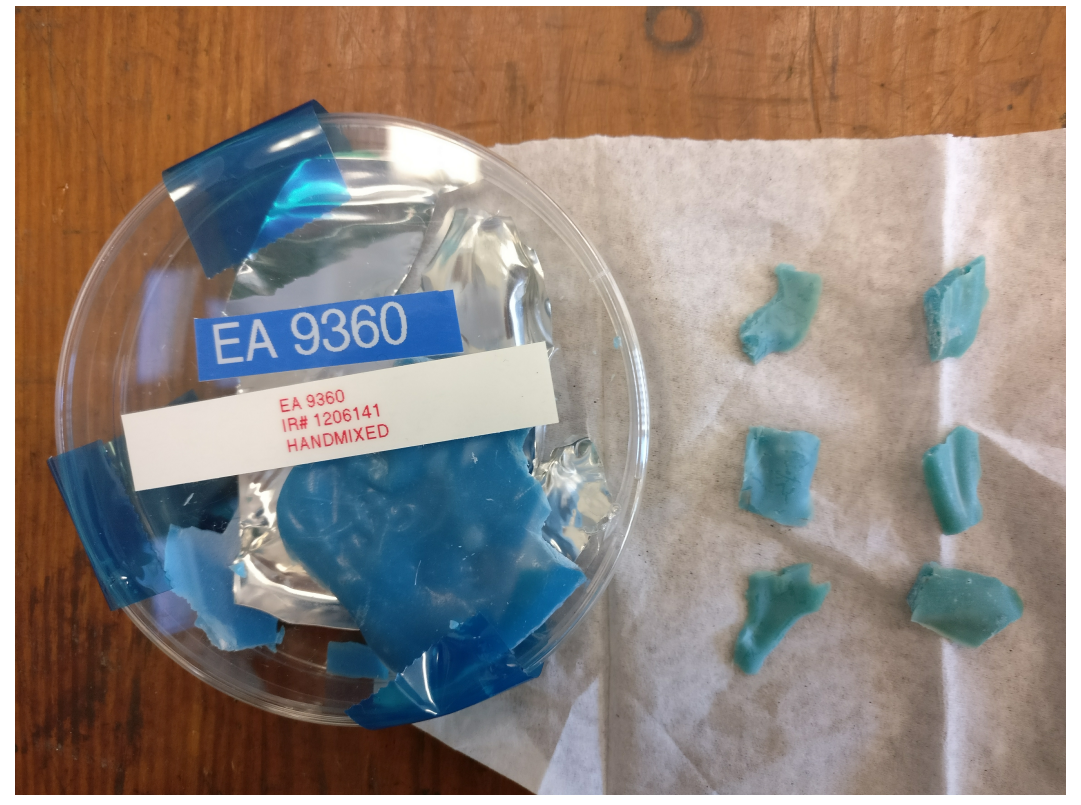

Figure B.8: EA 9360 Loctite adhesive samples untested (left), outgassed and AO exposed (center column), and outgassed (right column)

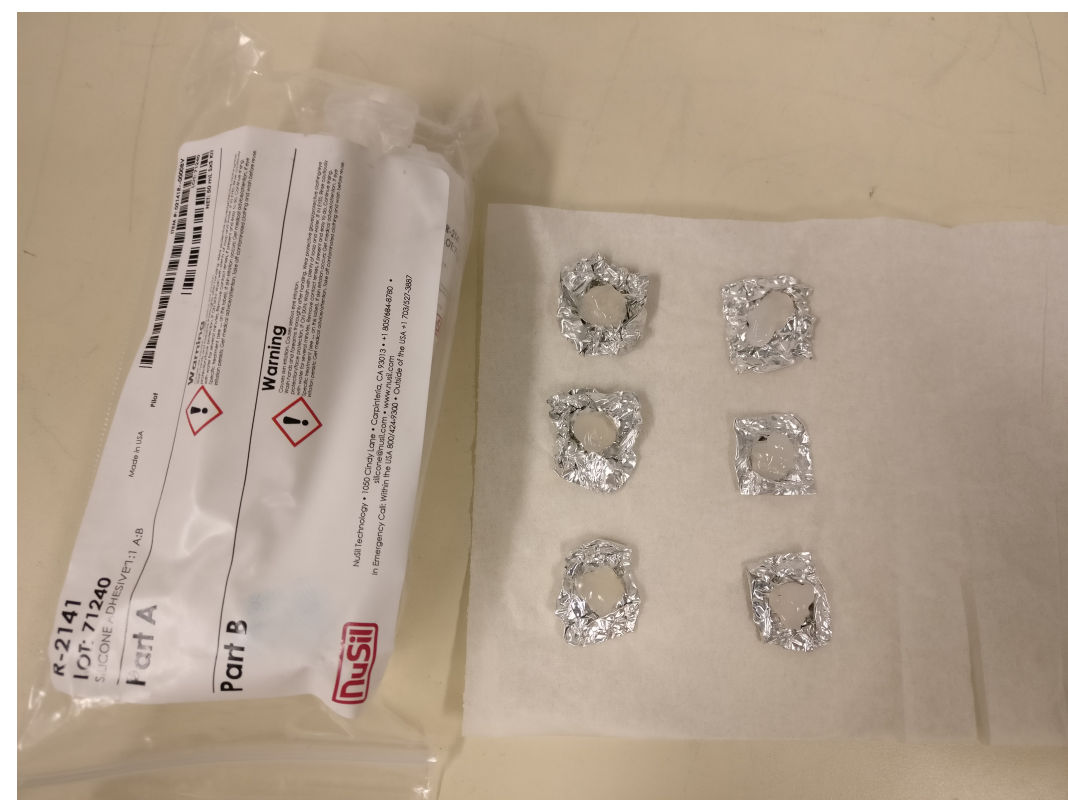

Figure B.9: NuSil R-2141 Silicone two-part adhesive samples original uncured material (left), outgassed and AO exposed (center column), and outgassed (right column) 
Appendix C

PROCEDURES

\section{C.1 MAX Sample Preparation Procedure}

1. Wear nitrile gloves whenever handling samples

2. Cut one kapton $\mathrm{HN}$ witness sample into $2 \times 2$ inch square. Do this for test samples as well if they are thin enough to fit under the cover plate.

3. Outgas all samples for a minimum of 48 hours below 200 mTorr

4. Remove samples from outgassing chamber and weigh within 5 minutes

5. Place witness sample on the ground plate over one of the four circular wear marks between the eight screw holes. Do this for test samples as well if they are thin enough to fit under the cover plate.

6. Cover the samples with the sample containment cover plate making sure to align it properly by matching up the arrow on the top of the ground plate and bottom of the cover plate.

7. Secure the cover plate in place with the eight low-profile $\frac{1}{4} "-20$ screws with the short screw used in the forward most hole to avoid interference with the copper ground strap below the ground plate.

8. Place thicker samples and abnormally shaped samples on top of the cover plate.

9. Ensure thicker samples that are made of conductive metals or that have conductive metal coatings sit flush with the cover plate. 
10. Replace the kapton tape covering the two ends of the bolts protruding from the DSS to the test section area.

\section{C.2 MAX Pump Down Procedure}

1. Ensure that all vacuum control panel toggles are switched to the "off" position

2. Ensure the main $3 \Phi$ power cable from the back of MAX is plugged in to a 208 $3 \Phi$ VAC breaker and rotated about 45 degrees clockwise.

3. Ensure the $2083 \Phi$ VAC breaker is flipped to the "on" position

4. Ensure the ball valve to the pressurized air line on the back of MAX is in the open position

5. Ensure the pressurized air regulator reads between $70-75$ psi

6. Flip the Main Power switch on the front control panel to the "on" position

7. Turn on the Granville-Phillips 316 Vacuum Gauge Controller; convectron gauge 3 (CG3) indicates chamber pressure in Torr

8. Use the hoist switch to lower the lid down into contact with the cylinder plexiglass chamber wall and ensure a proper seal.

9. Ensure all ports are closed, including the black nupro valve on the gas insertion line as well as the chamber vent valve.

10. Flip the Mechanical Pump Power switch on the front control panel to the "on" position

11. Press the green square "ON" button on the mechanical pump control box 
12. Flip the Mechanical Pump and Chamber Rough Valve switches to the "on" position

13. Monitor the chamber pressure on the Granville-Phillips 316 Vacuum Gauge Controller as the chamber pumps down

\section{C.3 MAX Operation Procedure}

1. Pump down the chamber as described in Appendix C.2

2. Connect the orange RF power BNC cable to the feedthrough port on the chamber lid

3. Allow the pump to run for $20-30$ minutes until the chamber pressure stabilizes

4. Open the black nupro valve on the gas insertion line; use the lever arm to adjust the pressure in the chamber to $175 \pm 10$ mTorr. Monitor the chamber for $1-2$ minutes to ensure it is stable.

5. Turn on the R301 generator

6. Set the power to 125 Watts

7. Turn on the MC2 controller

8. Switch to manual adjustment mode and adjust the load and tune capacitors to $50 \%$

9. Switch the adjustment mode back to automatic for both tune and load

10. Ensure chamber pressure is still at $175 \pm 10$ mTorr and adjust if necessary

11. Turn on the RF power using the button on the R301 
The capacitors on the MC2 should auto adjust and find a stable operational point where the reflected power is 1 or 0 Watts. If there is still reflected power, manually adjust tune and/or load until the reflected power is 0 Watts. If the capacitors motors begin to oscillate or if the system cannot be adjusted to get the reflected power to 0 Watts within 1 minute, turn off the system and refer to the MC2 manual.

12. Once a stable point has been achieved, adjust the phase and magnitude potentiometers on the left hand side of the AT3 until the MC2 controller shows both phase and magnitude at $0 \pm 25 \mathrm{mV}$. Note, a clockwise rotation will decrease the value.

13. Maintain a reflected power of 0 Watts and a pressure of $175 \pm 10$ mTorr. Record temperature values of the ground plate every hour.

\section{C.4 MAX Shut-Down Procedure}

1. Turn off the RF power using the button on the R301 generator

2. Close the black nupro valve on the gas insertion line

3. Turn off the R301 power supply and MC2 controller

4. Let the samples sit in the chamber under vacuum until the ground plate temperature is less than $35^{\circ} \mathrm{C}$

5. Flip the Chamber Rough Valve and Mechanical Pump switches on the front control panel to the "off" position

6. Flip the Vent switch on the front control panel to the "on" position and fully open the black nupro valve on the gas insertion line. When the chamber pressure reaches 700 Torr, flip the Vent switch the the "off" position. 
It is important to flip the Vent switch to the off position when the chamber reaches about 700 Torr otherwise the compressed air line will continue to overpressurize the chamber and cause the lid to suddenly lift off of the chamber making a loud "pop" sound in the process. Use the gas insertion line as an equalizer to allow the chamber to reach equilibrium pressure with the environment.

7. Disconnect the orange RF power BNC cable from the feedthrough port on the chamber lid.

8. Use the Hoist switch to raise the lid being careful to hold the orange RF power BNC cable out of the way so that minimal strain is put on it. Do not raise the lid so far that it strains the green ground cables connected to the chamber lid.

9. Remove any samples on top of the cover plate, remove the cover plate, and remove the kapton witness sample along with any samples that were under the cover plate.

Caution: the DSS will be very hot, avoid contact with the DSS. The ground plate will also be quite warm, use heavy duty work gloves to handle the cover plate and the $8 \frac{1}{4} "-20$ screws. Use Teflon tipped foreceps to handle any samples.

10. Weigh all samples within 5 minutes

11. Return the lid to the closed position when not in use

\section{C.5 ELI Sample Preparation}

Note: This procedure has been adapted from ASTM E-595 and from the outgassing test procedure from NASA JPL in order to suit the capabilities of the facilities in the Cal Poly Space Environments Lab

1. Wear nitrile gloves when handling samples and disks 
2. Clean the collector disks, copper sample bar, separator plate, and cold plate with a 50:50 mixture of acetone and ethanol

3. Dry out appropriate number of collector discs along with the entire test stand at $125 \pm 5^{\circ} \mathrm{C}$ and $<1 \mathrm{mTorr}$ for a minimum of 16 hours; keep collector disks in dessicator for 24 hours after bake out

4. Cut samples according to ASTM E595 depending on what type of material is to be tested; samples should be between 200 - $300 \mathrm{mg}$ if possible

5. Add samples to aluminum boats and place them in humidity controlled box at $50 \pm 5 \%$ relative humidity for at least 24 hours

6. Weigh the samples and $1.5 \times 1.5$ in UHV foil squares with a balance having $\pm 1 \mu g$ sensitivity; ensure the scale is level by checking the bubble level built into the top of the scale and adjust the scale legs if necessary

7. Wrap each collector disk in one square of UHV foil

8. Place the collector disks on the cold plate of the apparatus, clamped down between one spring loaded screw and two threaded immobile screws; ensure the foil side of the collector disk is facing the sample containment bar

9. Place the test samples in the sample compartments within the copper sample containment bar

10. Screw the cover plates onto their respective compartments with the end of a thermocouple pinched between the sample bar and each cover plate, making sure not to tighten the screws too much so that the covers stay flush with the bar 


\section{C.6 ELI Pump Down Procedure with Cryo Pump Off}

1. Use the Hoist switch on the front control panel to lower the bell jar and seal the chamber closed; ensure proper seal

2. Ensure all valves are shut (rough valve, vent valve, black nupro valve for $N_{2}$ gas insertion, etc.)

3. Turn on convectron gauge controller and cryo pump temperature monitor

4. Flip the Mechanical Pump switch on the front control panel to the "on" position to turn on the mechanical pump

5. Open the chamber rough valve by rotating the Chamber Rough nob counterclockwise many turns until resistance is met

6. Monitor the chamber pressure as the chamber pumps down

7. When the chamber reaches a low pressure $(50-150 \mathrm{mTorr})$ open the gate valve by rotating the Gate Valve handle counterclockwise many turns until resistance is met

8. Allow the mechanical pump to rough out the chamber and the cryo pump to a low pressure (150 mTorr is acceptable, 50 mTorr is ideal)

9. Turn on the compressor and cryo pump by flipping both switches on the front of the compressor unit to the "on" position. Note: Do not operate the cryo pump at a pressure above 150 mTorr. It is only ok for the chamber pressure to be above 150 mTorr when the gate valve is sealed and the cryo pump pressure is below 150 mTorr (unless the cryo is off).

10. When the cryo cools below $120 \mathrm{~K}$, close the chamber rough valve 
11. Turn off the mechanical pump by flipping the Mechanical Pump switch on the front control panel to the off position

12. Allow the cryo temperature to drop to $10-20 \mathrm{~K}$

Ensure the chamber pressure does not rise above 150 mTorr during cryo cooldown process

13. Turn on the ion gauge controller once the convectron gauge reaches $1-4$ mTorr and stops changing

\section{C.7 ELI Pump Down Procedure with Cryo Pump On}

1. Use the Hoist switch on the front control panel to lower the bell jar and seal the chamber closed; ensure proper seal

2. Ensure all valves are shut (rough valve, vent valve, black nupro valve for $N_{2}$ gas insertion, etc.)

3. Turn on convectron gauge controller and cryo pump temperature monitor

4. Flip the Mechanical Pump switch on the front control panel to the "on" position to turn on the mechanical pump

5. Open the chamber rough valve by rotating the Chamber Rough nob counterclockwise many turns until resistance is met

6. Monitor the chamber pressure as the chamber pumps down

7. When the chamber reaches a low pressure $(50-150 \mathrm{mTorr})$ open the gate valve by rotating the Gate Valve handle counterclockwise many turns until resistance is met

8. Close the chamber rough valve 
9. Turn off the mechanical pump by flipping the Mechanical Pump switch on the front control panel to the off position

10. Turn on the ion gauge controller once the convectron gauge reaches $1-4$ mTorr and stops changing

\section{C.8 ELI Operation Procedure}

1. Prepare and load samples as described in Appendix C.5

2. Close the ELI chamber and pump it down to $<5 x 10^{-} 5$ Torr (if possible) as described in Appendix C.6 or C.7

3. While the chamber is pumping down, turn on the Brinkmann MGW Lauda RM 20 water-cooling unit and set at temperature (typically $24^{\circ} \mathrm{C}$ ) to control the cold plate to $25 \pm 1^{\circ} \mathrm{C}$

4. When the chamber has reached $5 x 10^{-} 5$ Torr, turn on the Variac power supply (usually set to $30 \mathrm{~V}$ ) to raise the sample containment bar temperature to $125 \pm$ $1^{\circ} \mathrm{C}$ within 1.5 hours.

5. Continue to monitor cold plate temperature and containment bar temperatures, making adjustments to the water-cooling unit and Variac power supply if/when necessary

6. 24 hours after the sample bar reaches $125 \pm 1^{\circ} \mathrm{C}$, shut down the chamber as described in Appendix C.9

\section{C.9 ELI Shut Down Procedure}

1. Turn off Variac power supply 
2. Turn off ion gauge controller

3. Close gate valve

4. Turn compressor off if cryo pump is to be allowed to warm up, otherwise keep compressor running

5. Set nitrogen pressure regulator to about 25 psi

6. Open black nupro valve for the $N_{2}$ gas insertion line to vent the chamber with nitrogen; allow the pressure to rise to $100-200$ mTorr

7. Allow the temperature of the sample containment bar to decrease under vacuum to $50^{\circ} \mathrm{C}$ (or whatever temperature is manageable to retrieve samples)

8. Open vent valve and completely vent the chamber; maintain clockwise pressure on the Gate Valve to keep it closed while venting as it usually requires an additional 45 degrees of rotation in order to keep it closed as the pressure reaches around $200-300$ Torr

9. Open chamber and remove samples from compartments using Teflon-tipped forceps and remove collector disks from cold plate

10. Place samples and disks in the dessicator

11. Turn off the Brinkmann MGW Lauda RM 20 water-cooling unit

12. Weigh samples and UHV foil squares within 30 minutes of removal (if possible)

\section{C.10 NASA JPL Modified ASTM E595 Procedure}

This procedure was developed between NuSil and NASA JPL for JPL to use to perform outgassing testing. It was used as a reference in this research for how certain steps of 
the official ASTM E595 procedure can be adapted or ignored, however this procedure was not followed as a test procedure during the experimentation

1. Wipe down all sample chambers with clean room q-tip and IPA, with an emphasis on the channels in the chambers

2. Wipe down separator plates and condenser bar with clean room wipes

3. Ultra-sonicate all small VCM pieces in acetone, followed by IPA, then bake dry at $>125^{\circ} \mathrm{C}$

4. Prepare UHV aluminum foil sample "boats"

(a) Wipe UHV foil with acetone followed by IPA using clean-room wipers

(b) Cut foil into $0.75 " \times 0.75 "$ squares

(c) Fold using VCM boat tool and store in desiccant chamber

5. Wipe UHV foil for CVCM collection (targets) with acetone followed by IPA using clean-room wipers and cut the foil into $1.375 " \mathrm{x} 1.375 "$ squares.

6. Place material samples into conditioning chamber at $50 \%$ humidity for i, 24 hours

7. Wipe the inside of each petri dish used to hold samples and UHV foil targets with a clean-room wipe and IPA

8. Randomly assign 3 chambers to use for each material, with 1 - 2 blanks for each heating bar.

9. Place an empty boat in a small petri dish labeled with the chambers chosen.

10. Place a blank target in a large petri dish labeled with the chambers chosen

11. Weigh samples, targets, and boats on the micro-balance scale 
(a) Turn on the microbalance and let equilibrate until symbols at top left of display disappear

(b) Weigh standard $300 \mathrm{mg}, 100 \mathrm{mg}$, and $30 \mathrm{mg}$ weights.

(c) Based on weight records, check the $300 \mathrm{mg}$ and $100 \mathrm{mg}$ weights are within $\pm 5 \mu \mathrm{g}$; the $30 \mathrm{mg}$ weight can be within $\pm 10 \mu \mathrm{g}$.

(d) If weights are varying, repeatedly weigh the standard weights until they are within range

(e) Weigh empty boats and empty targets

(f) Record date and approximate time for each set of weighings, alongside weights of standards at that time

(g) Place conditioned samples into empty boats, aiming for $200-300 \mathrm{mg}$ of mass per sample

(h) Weigh boats and samples immediately, ideally less than 2 minutes after samples are taken out of conditioning chambers

12. Fold clean aluminum foil targets over target disks

13. Screw target discs into place on condenser bar using thumbscrews; hold target discs by edges only with clean gloves.

14. Attach separator plates to condenser bar, followed by bolting heater bars in place

15. Place sample boats in heater bar chambers; cover each chamber with chromeplated copper cover, and secure using retaining thumb-bolt

16. Wipe off bottom of bell jar and area on base prior to installing

\section{Begin VCM run}


(a) Verify that the Bell Jar Vent Valve is in the closed position

(b) Turn on Main Power, followed by Computer Power, Meters

(c) Turn on computer data logging software

(d) verify that all of the meters are recording correctly prior to the next step

(e) Turn on Target Cooling and vacuum pumps

(f) After bell jar reaches sufficient vacuum pressure, turn on Bar 1 Heat and Bar 2 Heat

(g) 24 hour experiment begins when vacuum pressure at $5 E-5$ Torr and heaters are at $125^{\circ} \mathrm{C}$; software records start time automatically

\section{End VCM run}

(a) After the 24 hour experiment time has passed, turn off the Bar 1 Heat and Bar 2 Heat

(b) Turn off vacuum pumps

(c) Carefully turn Bell Jar Vent Valve to vent Nitrogen position. Carefully watch the pressure monitor to avoid over-pressurizing the bell jar

(d) Return Bell Jar Vent Valve to closed position until heater bars reduce to $50^{\circ} \mathrm{C}$, then turn Bell Jar Vent Valve to Vent Air position

(e) Once system is sufficiently cooled to $40^{\circ} \mathrm{C}$, remove bell jar and unload samples and targets back into petri dishes

19. Weigh samples, targets, and boats on the micro-balance scale

(a) Weigh the boats with samples in them once they are cooled down to approximately room temperature; do not delay on this measurement as many materials will begin to absorb water immediately

(b) Weigh targets 
(c) Inspect targets for visible signs of contamination (haze on surface, etc)

\section{C.11 Leak Detector Procedure}

Note: Contact Dr. Richard Savage in the Cal Poly BMED department or David Laiho for permission to borrow the leak detector.

1. Ensure chamber is at ambient pressure.

2. Locate the chamber roughing tube that connects to the mechanical pump beneath the ELI chamber.

3. Disconnect the mechanical pump and connect the port coming out of the top of leak detector to the chamber roughing tube using any available tubulation.

4. Ensure the chamber is closed.

5. Turn on the Varian leak detector using the power switch on the back of the device.

6. Wait for the device to finish the start-up sequence.

7. Press the Test button and the leak detector will pull a vacuum on the chamber. If there are no significant leaks in the connection to the chamber the "Test Port Pressure" and "Spec Tube Pressure" will fall into or below the orange ranges within a few minutes. Once this happens, the leak detector is ready to detect helium and the screen should read "Fine Test".

8. Connect a flow regulator to a helium tank.

9. Connect a stainless steel braided flexible hose to the flow regulator.

10. Connect a spray nozzle like the one shown in fig. C.1 to the other end of the hose. 


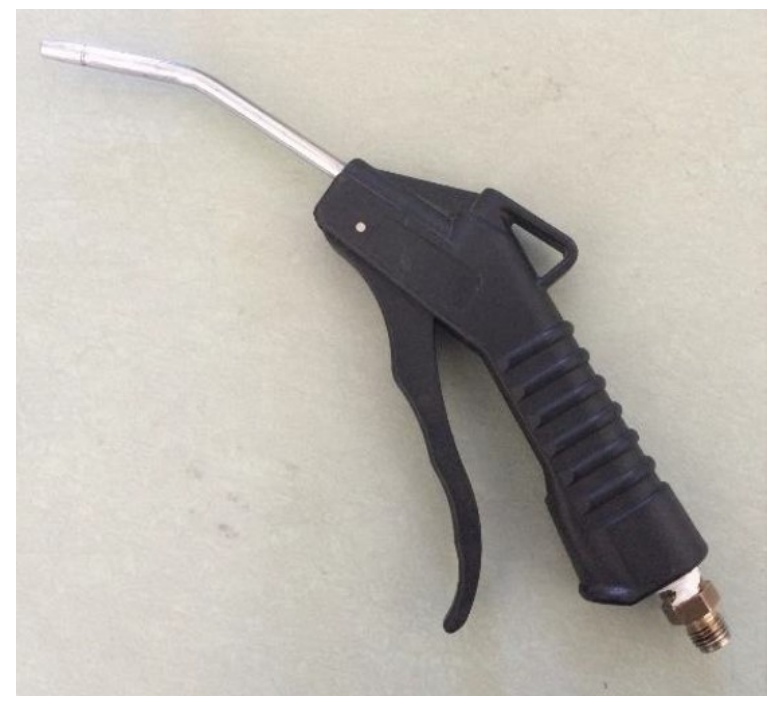

\section{Figure C.1: Spray Nozzle for use with Helium Leak Detector}

11. Open the valve on the top of the helium tank by rotating it counterclockwise a few turns.

12. Pressurize the hose with a very low psi by slowly turning the handle on the flow regulator clockwise until the needle just moves above zero psi.

13. Place the handle as close as possible to the suspected leak point and depress the spray nozzle handle briefly a small amount.

14. If possible, start at the top of the chamber and work down since helium rises.

15. Wait at least 10 seconds after each spray to see if the leak rate indicator registers an increase in helium detection.

16. If after a few minutes the leak detector's leak rate indicator does not return to the low level it was at originally, the device may be saturated with helium. In this case, the best way to reset it is to use the device to vent the chamber and re-pump it down again.

17. It can be difficult to pinpoint the exact leak once the general leak area is known. 
This is because all of the feedthroughs on ELI are on the bottom of the chamber and as the helium rises it spreads out on that surface underneath the chamber and penetrates many of the feedthroughs. It is most useful to create a makeshift weak seal around a feedthrough using a plastic bag, tape, or other container so that the container can be filled with helium from the bottom while the container prevents most of the helium from escaping to other areas.

18. Common sources of leaks on the ELI chamber include the gas and electrical feedthroughs that are not adhered to the chamber and any o-ring seals if they have aged and cracked.

\section{C.12 Cryo Pump Compressor Procedures}

\section{Compressor Fill Procedure}

Note: Page 45 in the CTI Crogenics manual in the lab has more details on this procedure. If the pressure is less than 30 psi the user must do the "Cryo Pump Decontamination" process described in the manual before operating the cyro pump or the compressor

(a) Safety glasses should be worn at all times during this process.

(b) Bring helium tank close to the compressor, attach a pressure regulator to the tank and a flexible braided stainless steel hose to the regulator.

(c) Ensure the regulator valve is closed by twisting it counterclockwise until it is loose, but not off.

(d) Open the tank valve by turning it counterclockwise a few turns.

(e) With the other end of the hose NOT attached to anything, slowly open the regulator valve by turning it clockwise until a small amount of helium 
can be heard escaping from the free end of the hose. Allow helium to bleed the line and remove any atmospheric air from it for 1 minute.

(f) While the line is bleeding, unscrew the brass cap on the helium nozzle on the back of the compressor next to the black nob labeled "GAS CHARGE".

(g) Keeping the helium on, attach the free end of the hose to the helium nozzle on the back of the compressor.

(h) Turn the regulator valve on the helium tank clockwise until the pressure in the line is $250 \mathrm{psi}$.

(i) Open the gas charge on the compressor by turning the black nob counterclockwise and allow the pressure in the tank to reach 200 psi before closing the gas charge.

(j) Close the tank valve by turning it clockwise until it is tight.

(k) Close the regulator valve by turning it counterclockwise until it is loose, but not off.

(l) Disconnect the hose from the compressor and the tank and replace the brass cap for the helium nozzle on the back of the tank.

\section{Compressor Drain Procedure}

(a) Remove the brass cap from the helium nozzle on the back of the compressor.

(b) Open the gas charge slowly by turning the black nob counterclockwise and allow the pressure to drop to 200 psi before closing the gas charge.

(c) Replace the brass cap from the helium nozzle.

3. Attaching and Removing the Lines from the Compressor to the Cryo Pump Note: Page 45 in the CTI Crogenics manual in the lab has more details on this 
procedure. Be sure not to over tighten the lines, just ensure the connection is snug.

Attach the lines in the following order:

(a) Connect the helium return line to the gas-return connector on the rear of the compressor unit

(b) Connect the helium supply line to the gas-supply connector on the rear of the compressor unit

(c) Connect the helium supply line to the gas-supply connector on the driveunit displacer assembly (on the cryo pump)

(d) Connect the helium return line to the gas-return connector on the driveunit displacer assembly (on the cryo pump)

\section{C.13 Anti-Static Gun Procedure}

Note: This procedure is not the manufacturer's official procedure for the Milty Zerostat 3 anti-static gun, it is the best working procedure as determined from experimentation.

1. Place the sample on the weighing pan of the scale using the Teflon-tipped forceps.

2. Close the draft shield and remove the glass top on the draft shield.

3. Remove the cap from the anti-stat gun and aim it down at the samples about 4-6 inches away.

4. Slowly pull the trigger all the way down to the handle and then slowly release the trigger while pointing at the sample.

5. Repeat this process as necessary until the scale drift is minimized. It may take a few repetitions before the static is neutralized. 
6. The gun works by spraying positively charged ions while pulling the trigger and it sprays negatively charged electrons while releasing the trigger. If you pull the trigger too fast you will hear a clicking sound which means you need to start the process over.

7. The device has 10,000 uses before it will need to be replaced and it can be tested by squeezing the trigger slowly with the cap on and looking for the LED in the cap to light up. 